\title{
Comparative Simulation Analysis of the Performance of the Logistics Manufacturing System at the Operative Level
}

\author{
Robert Bucki and Petr Suchánek (iD) \\ Department of Informatics and Mathematics, Silesian University in Opava, School of Business Administration in Karvina, \\ Karviná 73340, Czech Republic
}

Correspondence should be addressed to Petr Suchánek; suchanek@opf.slu.cz

Received 14 January 2019; Revised 27 March 2019; Accepted 1 April 2019; Published 7 May 2019

Academic Editor: Lucia Valentina Gambuzza

Copyright (C) 2019 Robert Bucki and Petr Suchánek. This is an open access article distributed under the Creative Commons Attribution License, which permits unrestricted use, distribution, and reproduction in any medium, provided the original work is properly cited.

\begin{abstract}
The article focuses on one of the current problems of manufacturing systems which consist of individual machines equipped with dedicated tools that are replaced when they are worn out. It is assumed that the machines are located within the reach of the robotic arm which carries out transport operations of semifinished products to designated production machines and storage containers in accordance with the production time period. The aim is to find such an arrangement of production activities, respectively, production paths for a given set of orders that will be effective from the time and cost point of view. Moreover, the whole issue is solved with regard to possible failures of individual stands, overfilling of some tanks, etc. The theory and practice of creating and using simulators as tools for the definition and verification of production plans are used to solve this issue. The starting point is the creation of a mathematical simulation model with the necessary but acceptable degree of simplification. The mathematical simulation model is tested on sample data in a feasibility study to perform a detailed usability analysis of the model. The output of the article is a simulation model for which, based on the analysis of simulation results, patterns of possible use in specific types of enterprises are given.
\end{abstract}

\section{Introduction}

A key trend in the area of business activities is the effort to optimise and thus increase the efficiency of individual processes. The reason for this is a long-established relationship between the efficiency of internal and external processes, their management and the competitiveness of the business [1]. The current rapid development of the business environment requires that process efficiency measurements and evaluations are carried out at increasingly shorter intervals and that the result of their optimisation meets the principles of a lean company concept [2] to comply with standard business process management approaches [3].

Analyses are performed using data and information that are the outputs of enterprise information systems. Not all of these systems are capable of providing the necessary information in sufficient quality and within a short time, and, in particular, knowledge of interrelationships and relationships about the current status of each business process, the enterprise as a whole, and enterprise behavior in the corporate environment. Companies are aware of this fact and therefore are willing to invest considerable funds in modern information systems and their modules [4]. As it can be seen from a wide range of sources and from real practice an adequate information system allowing a sufficient number of modern management support functionalities is one of the key conditions for promoting competitiveness [5] and after its introduction or implementation, its positive benefits can be observed in the first weeks after its introduction [6]. One of the conditions of a sufficiently strong competitiveness is meeting the needs of all business activities of the company in accordance with the principles of the so-called lean company concept [7] and constantly monitoring compliance with all its principles [8].

To sum up, we can say that choosing an appropriate information system has long been an important factor for businesses of all types. Due to the usually high investment in acquisition or expansion of the information system, it is 
necessary to pay close attention to the input analysis so that the resulting functionality meets all expectations [9]. Completely new requirements are emerging with the development of Industry 4.0. Automation, robotics, implementation of cobots, etc. require completely new approaches in the field of digitisation and management of enterprise informatics [10]. Also, business information systems must be tailored to this [11] as they are expected to have the highest possible degree of automation which is dependent on fast data processing [12]. This technological development necessitates a focus on human resources which must be sufficiently competent to be able to integrate into the company's comprehensive information system and respond in the right way at the right time [13]. The synergy of people and technology is the key prerequisite for the smooth running of the business. Therefore, an important aspect is the training of human resources, so that workers at all levels of the company (from employees to top management) are able to control, manage, and use new technologies [14]. A corporate information system as a comprehensive integrated business system can be described as optimal when the management system outputs show a high degree of efficiency, for example, through the appropriate sorting and ordering of orders, continuous production lines, correct production rerouting in the event of a machine failure, etc. This happens either automatically or depends on a human decision. Increasing decision-making efficiency can be achieved by implementing software modules to simulate system behavior after regulatory intervention [15]. Simulators, as we call these software modules, are a very powerful tool to reduce the failure of managerial decisions and thus reduce the costs that make bad decisions generally. Ideally, simulators are separate modules integrated into information systems so that they can easily define mathematical simulation models using current databases. Of course, it is also possible to implement the simulation approach in control systems with artificial intelligence support and fully automatic control systems [16] with which we will meet more and more often within Industry 4.0.

Based on the above, one of the key areas of informatics is the problem of creation of simulators. The main idea and goal is to create simulators that can be used to create plans and to validate these proposed plans before starting their subactivities. The basic starting point for creating a simulator is a mathematical model that represents, with a corresponding degree of simplification, the structural, functional and data architecture of the system [17]. In particular, in a manufacturing company, a simulation model can represent the flow of resources and products during the manufacturing process. It is advisable to implement the creation of a mathematical model of complex manufacturing system within a project whose starting point is a detailed feasibility study containing precise assumptions and specifications. The examples that can be found in professional and scientific sources include development of the mathematical model for the scheduling of production process and the allocation of the automatic guided vehicle in the flexible manufacturing system [18] or creating a mathematical programming model for a Kanban-controlled cellular manufacturing system with configuration capability [19]. The next step while creating a simulator is its programming followed by testing. Testing and functional verification of the simulator are important at every stage of the simulator's development. The above-mentioned feedback activities require adequate data, the source of which is the information systems of particular companies. The specific simulator procedure used in this article is presented in Section 3. A variety of methods, methodologies, and approaches can be used to create mathematical simulation models. The use of the process-oriented approach is presented by, e.g., $[20,21]$, whereas examples of value-oriented approach specifically for the supply chain modelling area are presented by, e.g., [22, 23]. Furthermore, there is a growing interest in the use of multiagent systems. The background to this topic is, inter alia, described in [24], the example in the form of negotiation model and tactics of manufacturing enterprise supply chain based on multiagent is presented in [25], and the example presenting agent-based modelling and simulation of decision-making behavior of e-retailers is shown in [26]. Another useful theme for modelling is using discrete or continuous Petri nets, for example, to model and simulate a logistics production system [27], discrete manufacturing systems [28], and flexible manufacturing systems [29]. Modelling and simulation are implemented in many cases to optimise the logistics system. A fuzzy approach can be used for the purposes of optimisation in relation to the problem of simulation presented, e.g., in [30], for a production system, e.g., in [31]. The heuristic approach appears to be effective in case of the so-called hard systems and can be applied, for example, in conjunction with time series characterising selected systems [32], for e-commerce processes [33], for resource planning and management needs in flexible manufacturing systems [34], or, for example, to optimise manufacturing and distributed systems [35]. Hybrid approaches for modelling and optimising manufacturing and other systems can be found, e.g., in [36-38], the use of neural networks, e.g., in [39]. It is possible to find the whole series of specific examples of theoretical background and examples of the so-called good practice. The essence is always to create corresponding models with simulation outputs usable for decision-making processes, preferably with the widest possible elements of intelligence [40].

The optimisation of production logistics as a complex of all direct and indirect activities of the company plays an increasingly important role in relation to the production of goods, production costs, used technologies, production organisation, etc. [41]. The diversity and complexity of production processes can be better modelled by providing an integrated view of different modelling approaches [42]. Logistics systems in manufacturing plants are often very extensive and their optimisation is conditioned by the evaluation of a number of parameters and their context. Specifically, when we focus on production logistics, which is the main subject of this article, we conclude that logistics financial costs are a budget cost item for all types of businesses. Therefore, the goal of every business is to minimise these costs. The aim of the article is to present a computer science approach to solving the problem of modelling a model company that works with random logistic costs, which alternate according to the current set of requirements flowing from individual 
orders. A heuristic approach is used for the solution of this model example which can be easily implemented to optimise the production logistics system. It is based on the experience of the authors who present examples of theoretical and practical bases, for example, in [43], [44], [45], or [46] who provides satisfactory decision support.

Logistics systems may be similar in nature; however, even a small difference in assumptions can lead to the need for preparing a new information solution to the problem of meeting customers' needs. Moreover, logistics systems are characterised by various limitations and their criteria are not often the same. These factors require autonomous solutions leading to forming new specification assumptions and a project resulting in offering the adequate simulator for solving specific logistics tasks.

Due to the fact that there are a number of different influencing factors in production and logistics systems the heuristic approach allows a number of experiments to be carried out on a continuous basis in order to achieve a satisfactory solution at the operation level which will ultimately result in a reduction in financial costs. The study presented in the article concerns the problem of manufacturing logistics which is important from the point of view of minimising manufacturing costs. This kind of approach is omnipresent in the contemporary world which is becoming increasingly globalised. Companies which either wish to continue to exist or become competitive need to implement more and more sophisticated solutions.

The main goal of the article is to introduce the production model of a production company usable for production strategy simulations. Heuristic algorithms that always determine a given production strategy are defined as part of the model. The goals of the paper also include preparing the specification assumptions and the model for creating a simulator of the complex manufacturing system. Subsequently, this model is to be implemented to carry out a series of simulation experiments which are then subject to the evaluation process in the case study section.

The article is divided into the main sections, i.e., Introduction, Methodology, Analysis, Results and Discussion, and Conclusion. The Introduction section presents the state-ofthe-art modelling and simulation of logistics systems. The Methodology section is devoted to presenting the way in which the paper was prepared in order to meet the research objectives. The Analysis section shows the mathematical model step by step as well as the control of the system. Finally, Results and Discussion section includes the study case where the way of simulating manufacturing processes is shown in detail which is followed by adequate discussion. The Conclusion section explains the research novelty, research limitations, contributions of the study for academics and practices, and recommendation for future studies.

\section{Methodology}

The article focuses on modelling the manufacturing system. The input of the model is the so-called order matrix in which orders from individual customers are stored. Each element of this matrix represents the customer and their specific requirement. Each order has predetermined resources. Resources are passed by a specified route in a production system assembled from a set of workstations performing specific operations on semifinished products and the final product is progressively made by individual machines wherein the manufacturing route is determined by a heuristic algorithm. There are storage capacities and storage facilities for intermediates, called buffers, along the production line. Buffer capacity is predefined and there is a possibility of temporary limitation of the site's operation in case of insufficient storage space. Although we consider series production, specifications, project, and subsequent software assume the possibility of extending the system to parallelarranged production lines. To satisfy the lean approach requirements, heuristic algorithms select a product to meet that criterion. The principle of operation forms the basis for creating a simulator of a modelled manufacturing system. However, there are manufacturing strategies that determine the starting point of creating an order matrix. In addition, costs are taken into account for demonstration needs. The costs are divided into those that can be strictly determined before the start of the production process and those that can be at least predicted before the manufacturing process.

To analyse the problem of minimising logistics costs it is necessary to follow the iterative model for creating the simulation tool. First of all, analysis of the real environment is the basis for the specification assumptions which lets the project of the simulator be prepared. Testing and subsequent validation of the simulator precede its implementation into the case study procedures. Figure 1 illustrates the research process of the study in detail.

The simulator of the logistics manufacturing system was designed in the form of three integrated modules: the data entry module, the simulation module, and the results module. In addition, each of the modules includes different working modes. The data entry module modes differ with the source of data; they can be input with a keyboard, generated randomly with or without the seed value or loaded directly from a file. The simulation module allows the operator of the system to perform a single simulation and experiments at random or with a set of heuristics as well as step-by-step simulations. The results module allows the operator to analyse and compare results depending on the number of performed simulations as well as their type. The simulator was written in the C\# programming language, using .NET Framework 4 and Microsoft Visual C\# 2012 Express programming environment. A DataGridFunctions library was created for the needs of the system. The library consists of two classes including the set of static methods working on arrays and DataGridView objects. It implements exception handling allowing an easier detection of incorrect input data. During the system testing the administrator and user manuals were created, including instructions for the installation process and the typical work with the system, showing its protection mechanisms against incorrect input data. Results of practical examples were analysed thoroughly. Moreover, they were confronted with the simulation results which allowed verifying the correctness of the system calculations. Testing the simulator required creating the set of initial data from the range of reasonable 


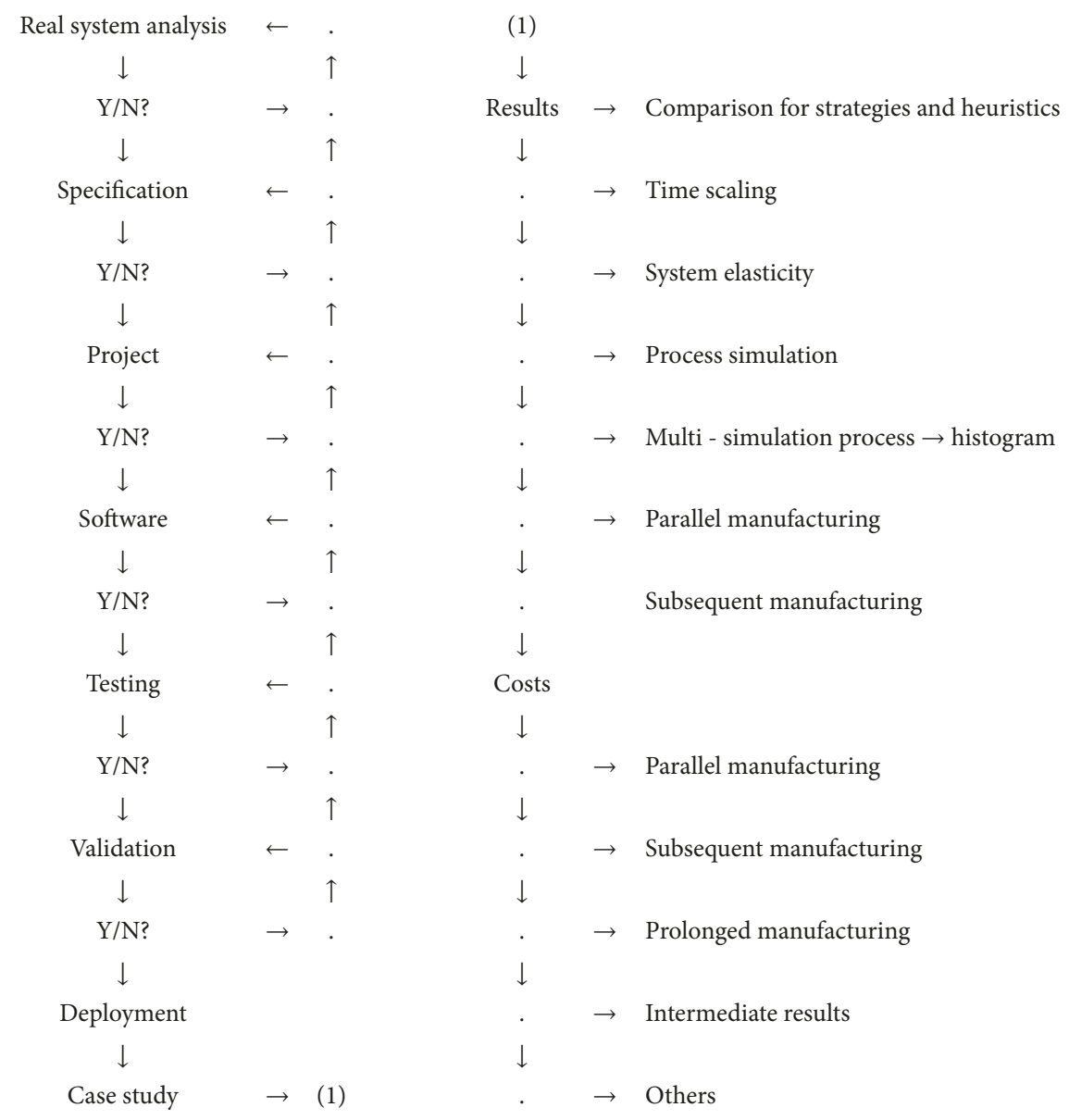

FIgURE 1: The iterative model for creating the simulator of the logistics system.

values based on thorough observations carried out in a few manufacturing plants. However, for the purpose of the simulation process there was a need for the initial data simplification. The verification result was positive so all the values returned by the system may now be considered to be correct. The data for the subsequent simulation process were generated within the assumed ranges. The data generation process can be replicated without any limitations. Once the data is generated it can be optionally modified in order to test alternative possibilities of making order matrix elements.

\section{Analysis}

3.1. Mathematical Model. Symbols used for logistic system modelling presented in this section are explained in detail in the symbol list at the end of this paper. They remain in accordance with the standards of mathematical models used for the description and simulation of logistics, production, trade and other systems.

It is assumed that the manufacturing system consists of the defined number of logistics manufacturing subsystems which are placed at various locations. Each $\pi$-th subsystem, $\pi=1, \ldots, \Pi$ is equipped with the same number of machines which use the same type of tools. Each $\pi$-th subsystem is also arranged in an identical way consisting of the charge zone, manufacturing hall, storing place for tools, storing place for semiproducts, and dispatching zone from which products are passed to the order matrix. It is also assumed that any element of the order matrix can be made on certain conditions in any manufacturing $\pi$-th subsystem if it is available at a required moment.

It is necessary to assume that customers set orders which are gathered together mathematically in the following matrix of orders (1):

$$
\begin{aligned}
& Z^{k}=\left[z_{m, n}^{k}\right], \\
& \quad m=1, \ldots, M, n=1, \ldots, N, k=0,1, \ldots, K
\end{aligned}
$$

where $z_{m, n}^{k}$ is the state of the $n$-th order set by the $m$-th customer at the $k$-th stage whereas $K$ is the total number of system decisions necessary to produce all order matrix elements approved for manufacturing at the stage $k=0$.

However, in a real manufacturing subsystem machines are placed at different locations within the range of the robotic arm in accordance with the predefined location arrangement. Each machine performs only one type of operation with the use of a predefined tool.

Charges of a certain type which are necessary for the manufacturing process are supplied on the demand basis with 
no delay. Each $n$-th product type, $n=1, \ldots, N$, is associated with a certain type of charge.

It is further assumed that there is the dedicated robotic arm in each $\pi$-th subsystem, $\pi=1, \ldots$, $\Pi$ which is responsible for the following operations:

(i) taking the charge material from the entrance gate of the logistics subsystem to the determined available machine;

(ii) transporting semiproducts from the preceding machine to the determined subsequent one;

(iii) transporting ready products to the dispatching gate respectively to the store for ready products; (iv) transporting semiproducts to their dedicated storing zone and back to the manufacturing zone;

(v) manipulating with semiproducts in their storing zone;

(vi) replacing a used tool with a new one.

The information point of view makes us present the sample manufacturing system in its simplified form in which each existing machine is to be associated with a certain defined point. This leads to creating the general matrix of structure of the manufacturing system which takes the following form:

$$
E=\left[e_{(m, n) /(i, j)}^{\pi}\right], \quad i=1, \ldots, I, j=1, \ldots, J, m=1, \ldots, M, n=1, \ldots, N, \pi=1, \ldots, \Pi
$$

where $e_{(m, n) /(i, j)}^{\pi}$ is a machine located in the $i$-th row of the $j$-th column in the $\pi$-th subsystem dedicated to performing a manufacturing operation on the $n$-th order for the $m$-th customer with the use of a certain type of tool.

At the same time, $e_{(m, n) /(i, j)}^{\pi}=1$ if the $n$-th order for the $m$-th customer can be made in the machine placed in the $i$-th row of the $j$-th column in the $\pi$-th subsystem; $e_{(m, n) /(i, j)}^{\pi}=0$ otherwise.

Let us present the matrix of adjustment of tools to operations in the following form:

$$
\mathrm{Y}=\left[v_{\alpha, \beta}\right], \quad \alpha=1, \ldots, A, \beta=1, \ldots, B
$$

where $v_{\alpha, \beta}$ represents the $\alpha$-th tool able to perform the $\beta$-th operation.

At the same time $v_{\alpha, \beta}=1$ if the $\alpha$-th tool can perform the $\beta$-th operation; $v_{\alpha, \beta}=-1$ otherwise.

It is assumed that a robotic arm is equipped with the required function enabling it to carry out the adequate operation. When a tool needs to be replaced, the machine is excluded from the manufacturing process for the time of replacement.
It is assumed that a certain machine is equipped with a dedicated tool only which is shown by means of the adjustment matrix (4):

$$
\mathrm{H}=\left[h_{i, j}^{\alpha}\right], \quad \alpha=1, \ldots, A, i=1, \ldots, I, j=1, \ldots, J
$$

where $h_{i, j}^{\alpha}$ is the adjustment of the $\alpha$-th tool to the machine placed in the $i$-th row of the $j$-th column.

At the same time, $h_{i, j}^{\alpha}=1$ if the adjustment is possible; $h_{i, j}^{\alpha}=-1$ otherwise.

Let us introduce the vector of buffer zones in the $\pi$-th manufacturing subsystem (5):

$$
\Xi^{\pi}=\left[\xi_{j}^{\pi}\right], \quad \mathrm{j}=1, \ldots, J, \pi=1, \ldots, \Pi
$$

where $\xi_{j}^{\pi}$ is the buffer zone behind the machine placed in the $j$-th column in the $\pi$-th manufacturing subsystem.

At the same time, $\xi_{j}^{\pi}=1$ if the buffer zone behind the machine in the $i$-th row of the $j$-th column which exists in the $\pi$-th manufacturing subsystem exists; $\xi_{j}^{\pi}=-1$ otherwise. Moreover, for calculation reasons it is assumed that the flow capacity of each buffer store is sufficient to ensure uninterrupted progress of the manufacturing process.

The matrix of production times in the $\pi$-th manufacturing system is introduced in

$$
T^{p r(\pi)}=\left[\tau_{(m, n) /(i, j)}^{p r(\pi)}\right], \quad i=1, \ldots, I, j=1, \ldots, J, m=1, \ldots, M, n=1, \ldots, N, \pi=1, \ldots, \Pi
$$

where $\tau_{(m, n) /(i, j)}^{p r(\pi)}$ is the production time of the $n$-th type of product for the $m$-th customer in the machine placed in the $i$-th row of the $j$-th column in the $\pi$-th subsystem.

The matrix of assembling times of tools in the $\pi$-th subsystem is introduced in

$$
\begin{aligned}
& T^{a s s e m b l(\pi)}=\left[\tau_{(i, j)}^{a s s e m b l(\pi)}\right], \\
& i=1, \ldots, I, j=1, \ldots, J, \pi=1, \ldots, \Pi
\end{aligned}
$$

where $\tau_{(i, j)}^{a \operatorname{assembl}(\pi)}$ is the assembling time of the tool in the machine placed in the $i$-th row of the $j$-th column in the $\pi$-th subsystem. 
The matrix of disassembling times of tools in the $\pi$-th subsystem is introduced in

$$
\begin{aligned}
T^{\operatorname{disas}(\pi)}=\left[\tau_{(i, j)}^{\operatorname{disas}(\pi)}\right], & \\
& \quad i=1, \ldots, I, \quad j=1, \ldots, J, \pi=1, \ldots, \Pi
\end{aligned}
$$

where $\tau_{(i, j)}^{\operatorname{disas}(\pi)}$ is the disassembling time of the tool in the machine placed in the $i$-th row of the $j$-th column in the $\pi$-th subsystem.

The matrix of transportation times for semiproducts from machines to the key manipulation point in the $\pi$-th subsystem is introduced in

$$
\begin{aligned}
T_{\longrightarrow}^{\operatorname{tr}(\pi)}=\left[\tau_{(i, j) / \longrightarrow}^{\operatorname{trl}(\pi)}\right] & \\
& \quad i=1, \ldots, I, j=1, \ldots, J, \pi=1, \ldots, \Pi
\end{aligned}
$$

where $\tau_{(i, j) / \longrightarrow}^{\operatorname{trl}(\pi)}$. is the transportation time of products from the machine placed in the $i$-th row of the $j$-th column to the key point in the $\pi$-th manufacturing system.

At the same time, $\tau_{(i, j) / \longrightarrow}^{\operatorname{trl}(\pi)}>0$ if there is a transport operation in the $\pi$-th manufacturing system; $\tau_{(i, j) / \longrightarrow}^{\operatorname{trl}(\pi)}=0$ otherwise.

The matrix of transportation times for semiproducts from the key point to machines in the $\pi$-th subsystem is introduced in

$$
\begin{aligned}
T_{\bullet}^{\operatorname{tr}(\pi)}=\left[\begin{array}{c}
\tau_{\bullet \longrightarrow /\left(i, j^{\prime}\right)}^{\operatorname{tr}(\pi)} \\
\longrightarrow
\end{array}\right. & \\
& i^{\prime}=1, \ldots, I, j^{\prime}=2, \ldots, J, \pi=1, \ldots, \Pi
\end{aligned}
$$

where $\tau_{\bullet \longrightarrow /\left(i, j^{\prime}\right)}^{t r(\pi)}$ is the transportation time of products from the key point to the machine placed in to the machine placed in the row $i$ of the column $j^{\prime}$ in the $\pi$-th manufacturing system, $j^{\prime} \neq j$. Moreover, in case of the product leaving column $j=5$ it is assumed it is directed to the key transport point before sending it to the store for ready products $\tau^{\operatorname{trl}(\pi)}$

$\left.\tau_{. \longrightarrow /\left(i, z_{s t}\right.}\right)^{t}$
The matrix of transportation times for semiproducts from machines to their buffer stores in the $\pi$-th subsystem is introduced in

$$
\begin{aligned}
T_{\longrightarrow \text { buffer }}^{\operatorname{tr}(\pi)}=\left[\tau_{(i, j) \longrightarrow b_{j}}^{\operatorname{tr}(\pi)}\right], & \\
& \quad i=1, \ldots, I, j=1, \ldots, J, \pi=1, \ldots, \Pi
\end{aligned}
$$

where $\tau_{(i, j) \longrightarrow b_{j}}^{\operatorname{tr}(\pi)}$ is the transportation time of a product from the machine placed in the $i$-th row of the $j$-th column to the $j$-th buffer store in the $\pi$-th manufacturing system.

If there is no transport operation carried out to the $j$-th buffer store due to its nonexistence then $\tau_{(i, j) \longrightarrow b_{j}}^{\operatorname{tr}(\pi)}=0$.

The matrix of transportation times for semiproducts from buffer stores to machines in the $\pi$-th subsystem is introduced in

$$
\begin{aligned}
& T_{\text {buffer } \rightarrow}^{t r(\pi)}=\left[\tau_{b_{j} \longrightarrow\left(i, j^{\prime}\right)}^{\operatorname{tr}(\pi)}\right], \\
& i=1, \ldots, I, j=1, \ldots, J, j^{\prime}=1, \ldots, J, \pi=1, \ldots, \Pi
\end{aligned}
$$

where $\tau_{b_{j} \longrightarrow\left(i, j^{\prime}\right)}^{\operatorname{tr}(\pi)}$ is the transportation time of a product from the $j$-th buffer store to machine placed in the $i$-th row of the column $j^{\prime}$ in the $\pi$-th manufacturing system.

If there is no transport operation carried out from the $j$-th buffer store due to its nonexistence then $\tau_{b_{j} \longrightarrow\left(i, j^{\prime}\right)}^{\operatorname{tr}(\pi)}=0$.

The matrix of unit transportation times for charge material from the store of charges to machines in the $\pi$-th subsystem is introduced in

$$
\begin{aligned}
T_{c h \longrightarrow}^{\operatorname{tr}(\pi)}=\left[\begin{array}{c}
\left.\tau_{c h \longrightarrow(i, j)}^{\operatorname{tr}(\pi)_{-}(m, n)}\right] \\
\end{array}\right. & \\
& \quad i=1, \ldots, I, j=1, \ldots, J, \pi=1, \ldots, \Pi
\end{aligned}
$$

where $\tau_{c h \longrightarrow(i, j)}^{\operatorname{tr}(\pi)_{-}(m, n)}$ is the unit transportation time of the charge material to the machine placed in the $i$-th row of the $j$ th column in case of making the $n$-th order for the $m$-th customer in the $\pi$-th manufacturing system.

If the charge material is not transported to the machine placed in the $i$-th row of the $j$-th column in case of making the $n$-th order for the $m$-th customer in the $\pi$-th manufacturing system then $\tau_{c h \longrightarrow(i, j)}^{t r(\pi) \_(m, n)}=0$.

The matrix of unit transportation times for ready products to the product store in the $\pi$-th subsystem is introduced in

$$
T_{\longrightarrow}^{\operatorname{tr}(\pi)}=\left[\tau_{s t}^{\operatorname{tr}(\pi)_{(}(m, n)}\right], \quad i=1, \ldots, I, j=1, \ldots, J, m=1, \ldots, M, n=1, \ldots, N, \pi=1, \ldots, \Pi
$$

where $\tau_{(i, j) \longrightarrow z_{s t}}^{\operatorname{tr}(\pi)_{-}(m, n)}$ is the unit transportation time of the ready product from the machine placed in the $i$-th row of the $j$-th column to the product store in case of making the $n$-th order for the $m$-th customer in the $\pi$-th manufacturing system.
If the ready product is not transported to the product store from the machine placed in the $i$-th row of the $j$ th column in case of making the $n$-th order for the $m$-th customer in the $\pi$-th subsystem then $\tau_{(i, j) \longrightarrow z_{s t}}^{t r(\pi)_{-}(m, n)}=0$. 
The matrix of times of storing charge materials in the $\pi$-th subsystem takes the following form:

$$
\begin{aligned}
& T_{w}^{(\pi) s t}=\left[\tau_{w /(m, n)}^{(\pi) s t}\right] \\
& m=1, \ldots, M, n=1, \ldots, N, w=1, \ldots, W, \pi=1, \ldots, \Pi
\end{aligned}
$$

where $\tau_{w /(m, n)}^{(\pi) s t}$ is the time of storing the $w$-th charge material for making the $n$-th product for the $m$-th customer in the charge store for the $\pi$-th plant.

The matrix of times of storing semiproducts in buffer stores in the $\pi$-th subsystem takes the following form:

$$
T^{(\pi) b u f \_s t}=\left[\tau_{m, n}^{(\pi) b u f \_s t}\right]
$$

where $\tau_{m, n}^{(\pi) b u f_{s} s t}$ is the average storing time of the $n$-th semiproduct made for the $m$-th customer in the available buffer store in the $\pi$-th manufacturing subsystem.

The matrix of times of storing ready products in the $\pi$-th subsystem takes the following form:

$$
T^{(\pi) s t_{-} z}=\left[\tau_{m, n}^{(\pi) s t_{-} z}\right]
$$

where $\tau_{m, n}^{(\pi) s t_{-} z}$ is the storing time of the $n$-th ready product made for the $m$-th customer in the ready product store of the $\pi$-th manufacturing system. in

The matrix of parallel manufacturing times is introduced

$$
\begin{array}{r}
T_{\mathrm{Q}_{-} \delta}^{\operatorname{par}(\pi)}=\left[\tau_{\mathrm{Q}_{-} \delta_{-}(m, n) /\left(m^{\prime}, n^{\prime}\right)}^{\operatorname{par}(\pi)}\right]=\left[\tau_{\mathrm{Q}_{-} \delta_{-}(m, n)}^{\operatorname{par}(\pi)_{\text {end }}}-\tau_{\mathrm{Q}_{-} \delta_{-}\left(m^{\prime}, n^{\prime}\right)}^{\text {par }(\pi)_{\text {start }}}\right], \\
\quad m=1, \ldots, M, n=1, \ldots, N, m^{\prime}=1, \ldots, M, n^{\prime}=1, \ldots, N \pi=1, \ldots, \Pi
\end{array}
$$

where

$$
\tau_{\mathrm{Q} \_\delta_{-}(m, n) /\left(m^{\prime}, n^{\prime}\right)}^{\operatorname{par}(\pi)} \text { is the parallel manufacturing time of }
$$
making the $n$-th order for the $m$-th customer and the $n^{\prime}$-th order for the $m^{\prime}$-th customer in the $\pi$-th subsystem with the use of the $\delta$-th criterion;

$\tau_{\mathrm{Q}_{-} \delta_{-}(m, n)}^{\text {par }(\pi) \text { start }}$ is the moment of beginning making the $n$ th order for the $m$-th customer and the $n^{\prime}$-th order for the $m^{\prime}$-th customer in the $\pi$-th subsystem with the use of the $\delta$-th criterion;

$\tau_{Q_{-} \delta_{-}(m, n)}^{\text {par }(\pi) \text { end }}$ is the moment of finishing making the $n$-th order for the $m$-th customer and the $n^{\prime}$-th order for the $m^{\prime}$-th customer in the $\pi$-th subsystem with the use of the $\delta$-th criterion.

At the same time if $n=n^{\prime}$ then $m \neq m^{\prime}$ and if $m=m^{\prime}$ then $n \neq n^{\prime}$. The matrix of times of replacement operations is introduced in

$$
\begin{aligned}
& T^{\alpha / \pi}=\left[\tau_{i, j}^{\alpha / \pi}\right], \\
& \alpha=1, \ldots, A, i=1, \ldots, I, j=1, \ldots, J, \pi=1, \ldots, \Pi
\end{aligned}
$$

where $\tau_{i, j}^{\alpha / \pi}$ is the replacement operation time of the $\alpha$-th tool in the machine placed in the $i$-th row of the $j$-th column in the $\pi$-th subsystem.

At the same time, $\tau_{i, j}^{\alpha / \pi}>0$ if the replacement operation of the $\alpha$-th tool in the machine placed in the $i$-th row of the $j$-th column in the $\pi$-th subsystem takes place; $\tau_{i, j}^{\alpha}=0$ otherwise.

Moreover, there is a need to introduce the matrix of transportation times of new tools which are stored in the storing place for tools in the $\pi$-th subsystem (20):

$$
\begin{aligned}
& T^{\alpha_{i n}}=\left[\tau_{i, j}^{\alpha_{i n} / \pi}\right], \\
& \alpha=1, \ldots, A, i=1, \ldots, I, j=1, \ldots, J, \pi=1, \ldots, \Pi
\end{aligned}
$$

where $\tau_{i, j}^{\alpha_{i n} / \pi}$ is the transportation time of the new $\alpha$-th type tool from the storing place to the machine placed in the $i$ th row of the $j$-th column in the $\pi$-th subsystem. At the same time, $\tau_{i, j}^{\alpha_{i n} / \pi}>0$ if the transportation to the discussed machine takes place; $\tau_{i, j}^{\alpha_{i n} / \pi}=0$ otherwise.

Additionally, there is a need to introduce the matrix of transportation times for worn out tools to the storing place for used tools in the $\pi$-th subsystem (21):

$$
\begin{aligned}
& T^{\alpha_{\text {out }} / \pi}=\left[\tau_{i, j}^{\alpha_{\text {out }} / \pi}\right] \\
& \alpha=1, \ldots, A, i=1, \ldots, I, j=1, \ldots, J, \pi=1, \ldots, \Pi
\end{aligned}
$$

where $\tau_{i, j}^{\alpha_{\text {out }}} / \pi$ is the transportation time of the worn out $\alpha$-th type tool from the machine placed in the $i$-th row of the $j$ th column to the storing place for worn out tools in the $\pi$-th subsystem. At the same time, $\tau_{i, j}^{\alpha_{\text {out }} / \pi}>0$ if the transportation from the discussed machine to the storing place for worn out tools takes place; $\tau_{i, j}^{\alpha_{\text {out }}} / \pi=0$ otherwise.

The base life matrix of tools used in the manufacturing system of a new brand set of tools used to manufacture elements of the order matrix is given in

$$
\begin{aligned}
G & =\left[g_{n /(i, j)}^{\alpha}\right] \\
i & =1, \ldots, I, j=1, \ldots, J, n=1, \ldots, N, \alpha=1, \ldots, A
\end{aligned}
$$

where $g_{n /(i, j)}^{\alpha}$ is the base number of units of the $n$-th product which can be manufactured in the machine in the $i$-th row of the $j$-th column before the $\alpha$-th dedicated tool is completely worn out and requires an immediate replacement. It is assumed that the life of a new tool is the same in each $\pi$-th subsystem.

Let us introduce the matrix of state of the $\pi$-th manufacturing subsystem (23): 


$$
S_{n}^{(\alpha / \pi) \downarrow k}=\left[s_{n /(i, j)}^{(\alpha / \pi) \downarrow}\right], \quad i=1, \ldots, I, j=1, \ldots, J, n=1, \ldots, N, \pi=1, \ldots, \Pi, k=0,1, \ldots, K
$$

where $s_{n /(i, j)}^{(\alpha / \pi) \downarrow k}$ is the number of units of the $n$-th product already made in the machine in the $i$-th row of the $j$-th column with the use of the $\alpha$-th tool in the $\pi$-th subsystem at the $k$-th state; if $s_{n /(i, j)}^{(\alpha / \pi)\lrcorner}=0$ the tool is regarded as brand new.
Let us introduce the capacity matrix of the $\pi$-th manufacturing subsystem (24):

$$
P_{n}^{(\alpha / \pi)\lrcorner k}=\left[p_{n /(i, j)}^{(\alpha / \pi)\rfloor}\right], \quad i=1, \ldots, I, j=1, \ldots, J, n=1, \ldots, N, \pi=1, \ldots, \Pi, k=0,1, \ldots, K
$$

where $p_{n /(i, j)}^{(\alpha / \pi)\rfloor}$ is the number of the $n$-th product units which still can be made in the machine in the $i$-th row of the $j$-th column with the use of the $\alpha$-th tool in the $\pi$-th subsystem at the $k$-th state. If the flow capacity of the machine does not allow to make the whole $n$-th unit, then $p_{n /(i, j)}^{(\alpha / \pi)\lrcorner k}=-1$. If there is any remaining flow capacity in the machine in the $i$-th row of the $j$-th column but the subsequent unit of the $n$-th product cannot be made fully in this machine in the $\pi$-th subsystem, then the replacement process is carried out automatically.

On the basis of the above assumptions we can determine the flow capacity of the machine in the $i$-th row of the $j$-th column in the $\pi$-th subsystem for the $n$-th element of the order matrix $Z^{k}(25)$ :

$$
p_{n /(i, j)}^{(\alpha / \pi)\rfloor k}=g_{n /(i, j)}^{(\alpha / \pi)}-s_{n /(i, j)}^{(\alpha / \pi)\rfloor k}
$$

Let us introduce the matrix of routes (for the case when the order of manufacturing operations is obligatory) (26):

$$
\begin{aligned}
D=\left[d_{(m, n), j}\right] & \\
j & =1, \ldots, J, m=1, \ldots, M, n=1, \ldots, N
\end{aligned}
$$

where $d_{(m, n), j}$ is the number of the $i$-th row through which the $n$-th semiproduct for the $m$-th customer is passed throughout the manufacturing process.

Throughout the course of manufacturing process the state of the system changes. Therefore, there is a need to analyse the state of the order matrix, tools in machines, charge materials, storing zones, etc. Equations of state illustrate changes in the manufacturing system after each decision made by the operator of the system.

The state of the order matrix changes after each production decision (27):

$$
Z^{0} \longrightarrow Z^{1} \longrightarrow \ldots \longrightarrow Z^{k} \longrightarrow \ldots \longrightarrow Z^{K}
$$

The order matrix is modified at each $k$-th stage as follows:

$$
z_{m, n}^{k}= \begin{cases}z_{m, n}^{k-1}-x_{m, n}^{k} & \text { if a certain number of units } x_{m, n}^{k} \text { of the } n \text {-th order for } \\ & \text { the } m \text {-th customer is made at the } k \text {-th stage, } \\ z_{m, n}^{k-1} & \text { otherwise. }\end{cases}
$$

The state of the $\pi$-th manufacturing system is subject to change if a production decision is made (29):

$$
S_{\pi}^{0} \longrightarrow S_{\pi}^{1} \longrightarrow \ldots \longrightarrow S_{\pi}^{k} \longrightarrow \ldots \longrightarrow S_{\pi}^{K}
$$

The state of the $\alpha$-th tool in the $i$-th machine in the $j$-th column changes in case of manufacturing the $n$-th product in the $\pi$-th subsystem:

$$
s_{n /(i, j)}^{(\alpha / \pi) \downarrow}= \begin{cases}s_{n /(i, j)}^{(\alpha / \pi)\rfloor}-s_{n /(i, j)}^{(\alpha / \pi) \downarrow k} & \text { if a certain number of units } x_{n /(i, j)}^{(\alpha / \pi)\lrcorner k} \text { of the } n \text {-th } \\ & \text { order is made in the } i \text {-th machine in the } j \text {-th } \\ & \text { column with the use of the } \alpha \text {-th tool in the } \pi \text {-th } \\ & \text { subsystem at the } k \text {-th state, } \\ s_{n /(i, j)}^{(\alpha / \pi) \downarrow} & \text { otherwise. }\end{cases}
$$


The state of the machine in the $i$-th row in the $j$-th column changes in case of replacement as follows:

$$
s_{n /(i, j)}^{(\alpha / \pi) k}= \begin{cases}s_{n /(i, j)}^{(\alpha / \pi)-k} & \text { if there was no replacement activity in the } i \text {-th machine } \\ & \text { in the } j \text {-th column at the } k \text {-th state } \\ 0 & \text { otherwise. }\end{cases}
$$

Let us introduce the matrix of unit manufacturing costs in the $\pi$-th manufacturing subsystem (32):

$$
C_{m, n}^{p r(\pi)}=\left[c_{(m, n) /(i, j)}^{p r(\pi)}\right], \quad i=1, \ldots, I, j=1, \ldots, J, m=1, \ldots, M, n=1, \ldots, N, \pi=1, \ldots, \Pi
$$

where $c_{(m, n) /(i, j)}^{p r(\pi)}$ is the unit cost of manufacturing the $n$-th order for the $m$-th customer in the $j$-th machine in the $i$-th row in the $\pi$-th manufacturing subsystem.

The cost of manufacturing the order $z_{m, n}^{0}$ in the $\pi$-th manufacturing subsystem is calculated as follows:

$$
C_{m, n}^{\sum p r(\pi) \_0}=z_{m, n}^{0} \cdot \sum_{j=1}^{J} \sum_{i=1}^{I}\left(c_{(m, n) /(i, j)}^{p r(\pi)} \cdot \tau_{(m, n) /(i, j)}^{p r(\pi)}\right)
$$

Let us introduce the matrix of probability coefficients of failure of tools in the $\pi$-th manufacturing subsystem (34):

$$
\begin{aligned}
\Gamma^{f a i l(\pi)}=\left[\gamma_{m, n}^{f a i l(\pi)}\right], & \\
& m=1, \ldots, M, n=1, \ldots, N, \pi=1, \ldots, \Pi
\end{aligned}
$$

where $\gamma_{m, n}^{f a i l(\pi)}$ is the probability coefficient of failure of tools in case of making the $n$-th order for the $m$-th customer in the $\pi$-th manufacturing subsystem.

Let us introduce the matrix of possible maintenance costs due to failure of the $\pi$-th manufacturing subsystem (35):

$$
\begin{aligned}
C^{f a i l(\pi)}=\left[c_{m, n}^{f a i l(\pi)}\right], & \\
& m=1, \ldots, M, n=1, \ldots, N, \pi=1, \ldots, \Pi
\end{aligned}
$$

where $c_{m, n}^{f a i l(\pi)}$ is the unit cost of possible maintenance procedures due to failure of a tool while making the $n$-th order for the $m$-th customer in the $\pi$-th manufacturing subsystem.

The costs of possible failure maintenance in case of making the order $z_{m, n}^{0}$ in the $\pi$-th manufacturing subsystem are calculated as follows (36):

$$
C_{m, n}^{f a i l(\pi)_{-} 0}=z_{m, n}^{0} \cdot \gamma_{m, n}^{f a i l(\pi)} \cdot c_{m, n}^{\text {fail }(\pi)}
$$

The matrix of unit assembling costs of tools in the $\pi$-th manufacturing subsystem is introduced in

$$
\begin{aligned}
& C^{\operatorname{assebl}(\pi)}=\left[c_{i, j}^{\text {assembl }(\pi)}\right], \\
& i=1, \ldots, I, j=1, \ldots, J, \pi=1, \ldots, \Pi
\end{aligned}
$$

where $c_{i, j}^{\text {assembl }(\pi)}$ is the unit assembling cost of the tool in the machine placed in the $i$-th row of the $j$-th column in the $\pi$-th subsystem.

The assembling costs of tools in case of making the order $z_{m, n}^{0}$ in the $\pi$-th manufacturing subsystem are calculated as follows:

$$
C_{m, n}^{\text {assambl }(\pi)_{-} 0}=\sum_{j=1}^{J} \sum_{i=1}^{I} \tau_{i, j}^{\text {assembl }(\pi)} \cdot c_{i, j}^{\text {assembl( } \pi)}
$$

The matrix of unit disassembling costs of tools in the $\pi$-th manufacturing subsystem is introduced (39):

$$
\begin{aligned}
C^{\text {disa }(\pi)}=\left[c_{i, j}^{\text {disasl }(\pi)}\right], & \\
& \quad i=1, \ldots, I, \quad j=1, \ldots, J, \pi=1, \ldots, \Pi
\end{aligned}
$$

where $c_{i, j}^{\text {disasl }(\pi)}$ is the unit disassembling cost of the tool in the machine placed in the $i$-th row of the $j$-th column in the $\pi$-th subsystem.

The disassembling costs of tools in case of making the order $z_{m, n}^{0}$ in the $\pi$-th manufacturing subsystem are calculated as follows:

$$
C_{m, n}^{\text {disas }(\pi) \_0}=\sum_{j=1}^{J} \sum_{i=1}^{I} \tau_{i, j}^{\text {disas }(\pi)} \cdot c_{i, j}^{\text {disas }(\pi)}
$$

The matrix of unit transportation costs for semiproducts from machines to the key point in the $\pi$-th manufacturing subsystem is introduced in

$$
\begin{aligned}
C_{\stackrel{\operatorname{tr}}{\operatorname{tr}(\pi)}}^{\rightarrow}=\left[c_{(i, j) /}^{\operatorname{tr}(\pi)} \rightarrow \cdot\right. & \\
& \quad i=1, \ldots, I, j=1, \ldots, J, \pi=1, \ldots, \Pi
\end{aligned}
$$

where $c_{(i, j) / \rightarrow \text {. }}^{t r(\pi)}$ is the unit transportation time of products from the machine placed in the $i$-th row of the $j$-th column to the key point in the $\pi$-th subsystem. 
The transportation costs for semiproducts from machines to the key point in case of making the order $z_{m, n}^{0}$ in the $\pi$-th manufacturing subsystem are calculated as follows:

$$
C_{(m, n) / \longrightarrow \bullet}^{t r(\pi) \_0}=\sum_{j=1}^{J} \sum_{i=1}^{I} \tau_{(i, j) / \longrightarrow \bullet}^{t r(\pi)} \cdot c_{(i, j) / \longrightarrow \bullet}^{t r(\pi)}
$$

The matrix of unit transportation costs for semiproducts from the key point in the $\pi$-th manufacturing subsystem is introduced in

$$
\begin{aligned}
C_{\bullet}^{\operatorname{tr}(\pi)} & =\left[\begin{array}{c}
c_{\bullet}^{\operatorname{tr}(\pi)} \longrightarrow\left(i, j^{\prime}\right)
\end{array}\right], \\
& \quad{ }^{\prime}=1, \ldots, I, j^{\prime}=2, \ldots, J, \pi=1, \ldots, \Pi
\end{aligned}
$$

where $c_{\bullet \longrightarrow\left(i, j^{\prime}\right)}^{\operatorname{tr}(\pi)}$ is the unit transportation cost of products from the key point to the machine placed in placed in the row $i$ of the column $j^{\prime}$ in the $\pi$-th subsystem, $j^{\prime} \neq j$.

The transportation costs for semiproducts from the key point to machines in case of making the order $z_{m, n}^{0}$ in the $\pi$ th manufacturing subsystem are calculated as follows:

$$
C_{(m, n) / \bullet \longrightarrow}^{t r(\pi) \_0}=\sum_{j=1}^{J} \sum_{i=1}^{I} \tau_{(i, j) / \bullet \longrightarrow}^{t r l(\pi)} \cdot c_{(i, j) / \bullet \longrightarrow}^{\operatorname{tr}(\pi)}
$$

The matrix of unit transportation costs for semiproducts transported from machines to buffer stores in the $\pi$-th manufacturing subsystem is introduced in

$$
\begin{aligned}
& C_{\longrightarrow \text { buffer }}^{t r(\pi)}=\left[\begin{array}{c}
c_{(i, j) \longrightarrow b_{j}}^{t r(\pi)}
\end{array}\right] \\
& i=1, \ldots, I, j=1, \ldots, J, \pi=1, \ldots, \Pi
\end{aligned}
$$

where $c_{(i, j) \longrightarrow b_{j}}^{\operatorname{tr}(\pi)}$ is the unit transportation cost of a product transported from the machine placed in the $i$-th row of the $j$-th column to the $j$-th buffer store in the $\pi$-th subsystem.

The transportation costs for semiproducts transported to buffer stores from machines in case of making the order $z_{m, n}^{0}$ in the $\pi$-th manufacturing subsystem are calculated as follows:

$$
C_{\stackrel{\text { buffer }}{\operatorname{tr}(\pi) \_0}}^{\longrightarrow} \sum_{j=1}^{J} \sum_{i=1}^{I} \tau_{(i, j) \longrightarrow b_{j}}^{\operatorname{tr}(\pi)} \cdot c_{(i, j) \longrightarrow b_{j}}^{\operatorname{tr}(\pi)}
$$

The matrix of unit costs of storing charge materials in the $\pi$-th manufacturing subsystem takes the following form:

$$
C_{s t}^{(\pi) w}=\left[c_{w /(m, n)}^{(\pi) s t}\right]
$$

where $c_{w /(m, n)}^{(\pi) s t}$ is the storing cost of the $w$-th charge material for making the $n$-th product for the $m$-th customer in the machine placed in the $\pi$-th manufacturing subsystem.

The matrix of unit costs of storing semiproducts in the buffer stores in the $\pi$-th manufacturing subsystem takes the following form:

$$
C^{(\pi) b u f_{s} s t}=\left[c_{m, n}^{(\pi) b u f_{s} s t}\right]
$$

where $c_{m, n}^{(\pi) b u f_{-} s t}$ is the storing cost of the $n$-th semiproduct made for the $m$-th customer in the available buffer stores in the $\pi$-th manufacturing subsystem.

Costs of storing semiproducts in the buffer stores in the $\pi$-th manufacturing subsystem can be presented as follows:

$$
C_{\max }^{(\pi) b u f_{s} s t}=\sum_{n=1}^{N} \sum_{m=1}^{M} z_{m, n}^{0} \cdot c_{m, n}^{(\pi) b u f_{s} s t} \cdot \tau_{m, n}^{(\pi) b u f_{s} s t}
$$

The matrix of unit costs of storing ready products in the $\pi$-th manufacturing subsystem takes the following form:

$$
C^{(\pi) s t_{-} z}=\left[c_{m, n}^{(\pi) s t_{-} z}\right]
$$

where $c_{m, n}^{(\pi) s t z}$ is the unit storing cost of the $n$-th ready product made for the $m$-th customer in the ready product store in the $\pi$-th manufacturing subsystem.

The total storing costs in the ready product store in the $\pi$-th manufacturing subsystem are presented as follows:

$$
C^{(\pi) \text { storage }}=\sum_{n=1}^{N} \sum_{m=1}^{M}\left(\tau_{m, n}^{(\pi) s t_{\_} z} \cdot c_{m, n}^{(\pi) s t_{-} z}\right)
$$

The matrix of maintenance costs in the $\pi$-th manufacturing subsystem takes the following form:

$$
\begin{aligned}
C^{(\pi) \text { main }}=\left[c_{i, j}^{(\pi) \text { main }}\right], & \\
& \quad i=1, \ldots, I, j=1, \ldots, J, \pi=1, \ldots, \Pi
\end{aligned}
$$

where $c_{i, j}^{(\pi) \text { main }}$ is the maintenance cost of the machine placed in the $i$-th row of the $j$-th column in the $\pi$-th manufacturing subsystem (per one unit of the order made in this machine).

The matrix of standstill costs in the $\pi$-th manufacturing subsystem takes the following form:

$$
\begin{aligned}
C^{(\pi) \text { still }}=\left[\mathcal{C}_{(\mathrm{i}, \mathrm{j}) /(\mathrm{m}, \mathrm{n})}^{(\pi) \text { still }}\right] & \\
& \quad i=1, \ldots, I, j=1, \ldots, J, \pi=1, \ldots, \Pi
\end{aligned}
$$

where $c_{(\mathrm{i}, \mathrm{j}) /(\mathrm{m}, \mathrm{n})}^{(\pi) \text { still }}$ is the unit standstill costs in case of making the $n$-th product for the $m$-th customer in the machine placed in the $i$-th row of the $j$-th column in the $\pi$-th manufacturing subsystem (per one unit of the order matrix).

The matrix of costs of implementing heuristic algorithms in the $\pi$-th manufacturing subsystem takes the following form:

$$
C^{(\pi)\lrcorner h e u r}=\left[\mathcal{C}_{(i, j) /(m, n)}^{(\pi) \text { heur }}\right]
$$

where $c_{(i, j) /(m, n)}^{(\pi) \text { heur }}$ is the cost of implementing heuristic algorithms in case of making the $n$-th product made for the $m$ th customer in the machine placed in the $i$-th row of the $j$-th column in the $\pi$-th manufacturing subsystem (per one unit of the order matrix).

The matrix of hidden operating costs in the $\pi$-th manufacturing subsystem takes the following form:

$$
C^{(\pi) \_h c}=\left[c_{(\mathrm{i}, \mathrm{j}) /(\mathrm{m}, \mathrm{n})}^{(\pi) \mathrm{hc}}\right]
$$


where $c_{(\mathrm{i}, \mathrm{j}) /(\mathrm{m}, \mathrm{n})}^{(\pi) \text { hc }}$ is the hidden unit cost in case of making the $n$-th product for the $m$-th customer in the machine placed in the $i$-th row of the $j$-th column of the $\pi$-th manufacturing subsystem (per one unit of the order matrix).

The matrix of unit transportation costs for semiproducts transported from buffer stores to machines in the $\pi$-th manufacturing subsystem is introduced in

$$
\begin{aligned}
& C_{b u f f e r \rightarrow}^{\operatorname{tr}(\pi)}=\left[\begin{array}{c}
c_{b_{j} \longrightarrow\left(i, j^{\prime}\right)}^{\operatorname{tr}(\pi)} \\
\text { (n) }
\end{array}\right], \\
& i=1, \ldots, I, j=1, \ldots, J, j^{\prime}=1, \ldots, J-1, \pi=1, \ldots, \Pi
\end{aligned}
$$

where $c_{b_{j} \longrightarrow\left(i, j^{\prime}\right)}^{\operatorname{tr}(\pi)}$ is the transportation cost of a product from the $j$-th buffer store to machine placed in the $i$-th row of the column $j^{\prime}$ in the $\pi$-th subsystem.

The unit transportation costs for semiproducts transported from buffer stores to machines in case of making the order in the $\pi$-th manufacturing subsystem are calculated as follows:

$$
C_{\text {buffer } \longrightarrow}^{\operatorname{tr}(\pi) \_0}=\sum_{j=1}^{J} \sum_{i=1}^{I} \tau_{b_{j} \longrightarrow\left(i, j^{\prime}\right)}^{\operatorname{tr}(\pi)} \cdot c_{b_{j} \longrightarrow\left(i, j^{\prime}\right)}^{\operatorname{tr}(\pi)}
$$

The matrix of unit transportation costs for the charge material from the store of charges to machines in the $\pi$-th manufacturing subsystem is introduced in

$$
\begin{aligned}
C_{c h \longrightarrow}^{\operatorname{tr}(\pi)}=\left[\begin{array}{c}
\left.c_{c h \longrightarrow(i, j)}^{\operatorname{tr}(\pi)_{2}(m, n)}\right] \\
{ }_{c} \longrightarrow
\end{array}\right. & \\
& \quad i=1, \ldots, I, j=1, \ldots, J, \pi=1, \ldots, \Pi
\end{aligned}
$$

where $c_{c h \longrightarrow(i, j)}^{\operatorname{tr}(\pi)_{-}(m, n)}$ is the unit transportation cost of the charge material to the machine placed in the $i$-th row of the $j$ th column in case of making the $n$-th order for the $m$-th customer in the $\pi$-th subsystem.
The total transportation costs of charge material transported from the charge store to machines in case of making the order $z_{m, n}^{0}$ in the $\pi$-th manufacturing subsystem are calculated as follows:

$$
C_{c h \longrightarrow}^{t r(\pi)_{-}(m, n)_{-} 0}=z_{m, n}^{0} \cdot \sum_{j=1}^{J} \sum_{i=1}^{I}\left(\tau_{c h \longrightarrow(i, j)}^{t r(\pi)_{-}(m, n)} \cdot c_{c h \longrightarrow(i, j)}^{t r(\pi)_{\_}(m, n)}\right)
$$

The matrix of unit transportation costs of ready products to the product store in the $\pi$-th manufacturing subsystem is introduced in

$$
\begin{aligned}
& C_{(i, j) \longrightarrow z_{s t}}^{t r(\pi)}=\left[c_{(i, j) \longrightarrow z_{s t}}^{\operatorname{tr}(\pi) \_(m, n)}\right], \\
& m=1, \ldots, M, n=1, \ldots, N, \pi=1, \ldots, \Pi
\end{aligned}
$$

where $c_{(i, j) \longrightarrow z_{s t}}^{\operatorname{tr}(\pi)_{-}(m, n)}$ is the unit transportation cost of the ready product transported the product store in case of making the $n$-th order for the $m$-th customer in the $\pi$-th subsystem.

The transportation cost of a ready product transported to the product store in case of making the order $z_{m, n}^{0}$ in the $\pi$-th manufacturing subsystem are calculated as follows:

$$
C_{(i, j) \longrightarrow z_{s t}}^{\operatorname{tr}(\pi)_{-}(m, n)_{0} 0}=z_{m, n}^{0} \cdot \sum_{j=1}^{J} \sum_{i=1}^{I}\left(\tau_{(i, j) \longrightarrow z_{s t}}^{t r(\pi)_{\_}(m, n)} \cdot c_{(i, j) \longrightarrow z_{s t}}^{t r(\pi)_{-}(m, n)}\right)
$$

The matrix of parallel manufacturing costs in the $\pi$-th manufacturing subsystem is introduced in

$$
C_{\mathrm{Q}_{-} \delta}^{\operatorname{par}(\pi)}=\left[c_{\mathrm{Q}_{-} \delta_{-}(m, n) /\left(m^{\prime}, n^{\prime}\right)}^{\operatorname{par}(\pi)}\right], \quad m=1, \ldots, M, n=1, \ldots, N, m^{\prime}=1, \ldots, M, n^{\prime}=1, \ldots, N \pi=1, \ldots, \Pi
$$

where $c_{Q_{-} \delta_{-}(m, n) /\left(m^{\prime}, n^{\prime}\right)}^{\operatorname{par}(\pi)}$ is the manufacturing cost of making the $n$-th order for the $m$-th customer and the $n^{\prime}$-th order for the $m^{\prime}$-th customer in a parallel way in the $\pi$-th subsystem with the use of the $\delta$-th criterion.

At the same time if $n=n^{\prime}$ then $m \neq m^{\prime}$ and if $m=m^{\prime}$ then $n \neq n^{\prime}$.

The matrix of costs of replacement operations in the $\pi$-th manufacturing subsystem is introduced in

$$
\begin{aligned}
& C^{\alpha / \pi}=\left[c_{i, j}^{\alpha / \pi}\right], \\
& \alpha=1, \ldots, A, i=1, \ldots, I, j=1, \ldots, J, \pi=1, \ldots, \Pi
\end{aligned}
$$

where $c_{i, j}^{\alpha / \pi}$ is the replacement operation cost of the $\alpha$-th tool in the machine placed in the $i$-th row of the $j$-th column in the $\pi$-th subsystem.
At the same time, $c_{i, j}^{\alpha / \pi}>0$ if the replacement operation of the $\alpha$-th tool in the machine placed in the $i$-th row of the $j$-th column in the $\pi$-th subsystem takes place; $c_{i, j}^{\alpha}=0$ otherwise.

The matrix of transportation costs for new tools which are stored in the storing place for tools in the $\pi$-th manufacturing subsystem takes the following form:

$$
\begin{aligned}
& C^{\alpha_{i n}}=\left[c_{i, j}^{\alpha_{i n} / \pi}\right] \\
& \alpha=1, \ldots, A, i=1, \ldots, I, j=1, \ldots, J, \pi=1, \ldots, \Pi
\end{aligned}
$$

where $c_{i, j}^{\alpha_{i n} / \pi}$ is the transportation cost of the new $\alpha$-th type tool from the storing place to the machine placed in the $i$-th row of the $j$-th column in the $\pi$-th subsystem. 
At the same time, $c_{i, j}^{\alpha_{i n} / \pi}>0$ if the transportation to the discussed machine takes place; $c_{i, j}^{\alpha_{i n} / \pi}=0$ otherwise.

The matrix of transportation costs for worn out tools to the storing place for used tools in the $\pi$-th manufacturing subsystem takes the following form:

$$
\begin{aligned}
& C^{\alpha_{\text {out }} / \pi}=\left[c_{i, j}^{\alpha_{\text {out }} / \pi}\right], \\
& \alpha=1, \ldots, A, i=1, \ldots, I, j=1, \ldots, J, \pi=1, \ldots, \Pi
\end{aligned}
$$

where $c_{i, j}^{\alpha_{\text {out }} / \pi}$ is the transportation cost of the worn out $\alpha$-th type tool from the machine placed in the $i$-th row of the $j$ th column to the storing place for worn out tools in the $\pi$-th subsystem. At the same time, $c_{i, j}^{\alpha_{\text {out }} / \pi}>0$ if the transportation from the discussed machine to the storing place for worn out tools takes place; $c_{i, j}^{\alpha_{\text {out }} / \pi}=0$ otherwise.

Let us introduce the cost of the replacement process in the $\pi$-th manufacturing subsystem (66):

$$
C_{r e p l}^{\pi}=\tau_{r e p l}^{\pi} \cdot c_{r e p l}^{\pi}
$$

where

$c_{r e p l}^{\pi}$ is the unit cost of the replacement process in the $\pi$-th manufacturing subsystem,

$\tau_{\text {repl }}^{\pi}$ is the total replacement time in the $\pi$-th manufacturing subsystem.

Let us introduce the cost of the lost flow capacity in the $\pi$-th manufacturing subsystem (67):

$$
C_{l f c}^{\pi}=\varsigma_{l f c}^{\pi} \cdot c_{l f c}^{\pi}
$$

where

$c_{l f c}^{\pi}$ is the unit cost of the lost flow capacity after completing the manufacturing process in the $\pi$-th manufacturing subsystem,

$\varsigma_{l f c}^{\pi}$ is the number of lost flow capacity units after completing the manufacturing process in the $\pi$-th manufacturing subsystem.

Let us introduce the fixed manufacturing cost in the $\pi$-th manufacturing subsystem (68):

$$
C_{f i x}^{\pi}=\frac{1}{\varsigma_{f i x}^{\pi}} \cdot \sum_{j=1}^{J} \sum_{i=1}^{I}\left(c_{(m, n) /(i, j)}^{p r(\pi)} \cdot \tau_{(m, n) /(i, j)}^{p r(\pi)}\right)
$$

where $\sum_{j=1}^{J} \sum_{i=1}^{I}\left(c_{(m, n) /(i, j)}^{p r(\pi)} \cdot \tau_{(m, n) /(i, j)}^{p r(\pi)}\right)$ is the unit cost of making the $n$-th order for the $m$-th customer in the $\pi$-th manufacturing subsystem; $\varsigma_{f i x}^{\pi}$ is the coefficient of stable costs in the $\pi$-th manufacturing subsystem.

For the purpose of the paper costs are divided into two sample groups:

(a) direct costs consisting of costs which can be calculated straight away as they depend on the amount of the order, i.e., costs of manufacturing, charge storing, charge transportation, ready product transportation, ready product storing, and parallel manufacturing, (b) indirect costs consisting including costs of failures, semiproduct storing in buffer stores, assembling of tools, replacement operations, semiproduct transportation into and out of buffer stores, replacement operations, transportation of a new tool in and out, maintenance, standstill, heuristic implementing, and hidden operations.

The approach cost of the $\pi$-th manufacturing subsystem is calculated as follows:

$$
\begin{aligned}
& C_{\mathrm{Q} \delta}^{\pi} \\
& =\sum_{n=1}^{N} \sum_{m=1}^{M} \sum_{j=1}^{J} \sum_{i=1}^{I} \tau_{(m, n) /(i, j)}^{p r(\pi)} \cdot c_{(m, n) /(i, j)}^{p r(\pi)} \\
& +\sum_{w=1}^{W} \sum_{n=1}^{N} \sum_{m=1}^{M} \tau_{w /(m, n)}^{(\pi) s t} \cdot c_{w /(m, n)}^{(\pi) s t} \\
& +\sum_{n=1}^{N} \sum_{m=1}^{M} \sum_{j=1}^{J} \sum_{i=1}^{I} \tau_{c h \longrightarrow(i, j)}^{t r(\pi)_{-}(m, n)} \cdot c_{c h \longrightarrow(i, j)}^{t r(\pi)_{(}(m, n)} \\
& +\sum_{n=1}^{N} \sum_{m=1}^{M} \sum_{j=1}^{J} \sum_{i=1}^{I} \tau_{(i, j) \longrightarrow z_{s t}}^{t r(\pi)_{-}(m, n)} \cdot c_{(i, j) \longrightarrow z_{s t}}^{t r(\pi)_{-}(m, n)} \\
& +\sum_{n=1}^{N} \sum_{m=1}^{M} \tau_{m, n}^{(\pi) s t_{-} z} \cdot c_{m, n}^{(\pi) s t z z} \\
& +\sum_{n^{\prime}=1}^{N} \sum_{m^{\prime}=1}^{M} \sum_{n=1}^{N} \sum_{m=1}^{M} \tau_{\mathrm{Q}_{-} \delta_{-}(m, n) /\left(m^{\prime}, n^{\prime}\right)}^{\operatorname{par}(\pi)} \cdot c_{\mathrm{Q}_{-} \delta_{-}(m, n) /\left(m^{\prime}, n^{\prime}\right)}^{\operatorname{par}(\pi)} \\
& +C_{r e p l}^{\pi}+C_{l f c}^{\pi}+C_{f i x}^{\pi}
\end{aligned}
$$

The company cost of the $\pi$-th manufacturing subsystem is calculated as follows:

$$
\begin{aligned}
& C_{f c}^{\pi}=\sum_{n=1}^{N} \sum_{m=1}^{M}\left(\gamma_{m, n}^{f a i l(\pi)} \cdot c_{m, n}^{f a i l(\pi)}+\tau_{m, n}^{(\pi) b u f_{\_} s t} \cdot c_{m, n}^{(\pi) b u f_{-} s t}\right) \\
& +\sum_{j=1}^{J} \sum_{i=1}^{I}\left(\tau_{(i, j)}^{a s s e m b l(\pi)}\right. \\
& \cdot c_{(i, j)}^{\operatorname{assembl}(\pi)}+\tau_{(i, j)}^{\operatorname{disas}(\pi)} \cdot c_{(i, j)}^{\operatorname{disas}(\pi)}+\tau_{(i, j) / \longrightarrow}^{\operatorname{tr}(\pi)} \cdot c_{(i, j) / \longrightarrow}^{\operatorname{tr}(\pi)} . \\
& \left.+\tau_{(i, j) / \longrightarrow b_{j}}^{\operatorname{tr}(\pi)} \cdot c_{(i, j) / \longrightarrow b_{j}}^{\operatorname{tr}(\pi)}\right) \\
& +\sum_{j^{\prime}=1}^{J} \sum_{i=1}^{I}\left(\tau_{\bullet\left(i, j^{\prime}\right)}^{\operatorname{tr}(\pi)}\right. \\
& \left.\cdot c_{\bullet \longrightarrow\left(i, j^{\prime}\right)}^{\operatorname{tr}(\pi)}+\tau_{b_{j} \longrightarrow(i, j)}^{\operatorname{tr}(\pi)} \cdot \tau_{b_{j} \longrightarrow\left(i, j^{\prime}\right)}^{\operatorname{tr}(\pi)}\right) \\
& +\sum_{j=1}^{J} \sum_{i=1}^{I} \sum_{\alpha=1}^{A}\left(\tau_{i, j}^{\alpha / \pi}\right. \\
& \left.\cdot c_{i, j}^{\alpha / \pi}+\tau_{i, j}^{\alpha_{\text {in }} / \pi} \cdot c_{i, j}^{\alpha_{\text {in }} / \pi}+\tau_{i, j}^{\alpha_{\text {out }}} / \pi \cdot c_{i, j}^{\alpha_{\text {out }} / \pi}\right)
\end{aligned}
$$




$$
\begin{aligned}
& +\sum_{j=1}^{J} \sum_{i=1}^{I} \tau_{i, j}^{(\pi) \text { main }} \\
& +\sum_{n=1}^{N} \sum_{m=1}^{M} \sum_{j=1}^{J} \sum_{i=1}^{I}\left(c_{(\mathrm{i}, \mathrm{j}) /(\mathrm{m}, \mathrm{n})}^{(\pi) \mathrm{till}}+c_{(i, j) /(m, n)}^{(\pi) h \text { hur }}+c_{(\mathrm{i}, \mathrm{j}) /(\mathrm{m}, \mathrm{n})}^{(\pi) h c}\right)
\end{aligned}
$$

The logistics costs of the $\pi$-th manufacturing subsystem are calculated as follows:

$$
C_{o p-(\mathrm{Q}-\delta)}^{\pi}=C_{\mathrm{Q}_{-} \delta}^{\pi}+C_{f_{c}}^{\pi}
$$

It can also be assumed that the longer the manufacturing process lasts the higher the costs can be generated increasing the base manufacturing costs $C_{\text {base } \delta}$ for the given $\delta$-th approach. These costs are the sum of manufacturing, parallel manufacturing, replacement and lost flow capacity costs for each $\delta$-th approach. The minimal manufacturing costs are sought for in accordance with the given criterion from the set of criteria also including approaches involving the use of one heuristic only. However, another manufacturing approach can generate lower costs but at the same time it does not meet other requirements such as the minimal manufacturing time, minimal replacement time and minimal lost flow capacity.

It is also assumed that prolonged manufacturing generates costs which can be presented by means of the matrix of prolonged manufacturing in the $\pi$-th subsystem (72):

$$
C_{\text {prol_ } \delta}^{\pi}=\Delta \tau_{\text {prol_ } \delta}^{\pi} \cdot c_{\text {prol }}^{\pi}
$$

where

$c_{\text {prol }}^{\pi}$ is the unit cost of prolonged manufacturing in the $\pi$-th subsystem.

$\Delta \tau_{\text {prol_s } \delta}^{\pi}$ is the time of prolonged manufacturing according for the $\delta$-th approach in the $\pi$-th subsystem.

At the same time,

$$
\Delta \tau_{\text {prol_ } \delta}^{\pi}=\tau_{\delta}^{\pi \_ \text {end }}-\min \tau_{\delta}^{\pi \_ \text {end }}
$$

where

$\min \tau_{\delta}^{\pi \text { end }}$ is the time of completing making the order by the manufacturing approach minimising the manufacturing time in the $\pi$-th subsystem;

$\tau_{\delta}^{\pi-e n d}$ is the time of completing making the order by the $\delta$-th approach in the $\pi$-th subsystem.

Each $\pi$-th manufacturing subsystem can be evaluated on the basis of many factors. For the purpose of the article flexibility of the $\pi$-th manufacturing subsystem is subject to analysis (74):

$$
l_{\text {flex }}^{\pi}=\sqrt{\iota_{\text {state }}^{\pi} \cdot l_{\text {usage }}^{\pi}}
$$

where

$\iota_{\text {flex }}$ is flexibility of the $\pi$-th manufacturing subsystem,

$\iota_{\text {state }}$ is state of the $\pi$-th manufacturing subsystem,

$\iota_{\text {usage }}$ is usage of the $\pi$-th manufacturing subsystem.
3.2. Control. To control the simulation process there is a need to take the following elements into account and combine them subsequently:

(1) heuristic algorithms;

(2) manufacturing strategies;

(3) control approaches.

3.2.1. Heuristic Algorithms. In order to choose an element of the matrix $Z$ and subsequently direct it to the manufacturing zone there is a need to choose one from a wide range of available heuristic algorithms. Sample heuristic algorithms are considered for demonstration purposes; however, their number may be much bigger.

Let $z_{\mu, \eta}^{k}$ be the element to be made in the manufacturing zone at the $k$-th stage, where $k=1, \ldots, K, 1 \leq \mu \leq M, 1 \leq$ $\eta \leq N$. On this basis the following heuristic algorithms are put forward:

$$
z_{\mu, \eta}^{k}=\left\{\begin{array}{lll}
\max z_{m, n}^{k} & \ldots & \text { (heuristic 1) } \\
\min z_{m, n}^{k} & \ldots & \text { (heuristic 2) } \\
\max \frac{z_{m, n}^{k}}{\sum_{m=1}^{M} \sum_{n=1}^{N} z_{m, n}^{k}} & \ldots & \text { (heuristic 3) } \\
\min \frac{z_{m, n}^{k}}{\sum_{m=1}^{M} \sum_{n=1}^{N} z_{m, n}^{k}} & \ldots & \text { (heuristic 4) } \\
\max \frac{z_{m, n}^{k}}{\sum_{m=1}^{M} z_{m, n}^{k}} & \ldots & \text { (heuristic 5) } \\
\min \frac{z_{m, n}^{k}}{\sum_{m=1}^{M} z_{m, n}^{k}} & \ldots & \text { (heuristic 6) } \\
\min \frac{z_{m, n}^{k}}{\sum_{n=1}^{N} z_{m, n}^{k}} & \ldots & \text { (heuristic 7) } \\
\min \frac{z_{m, n}^{k}}{\sum_{n=1}^{N} z_{m, n}^{k}} & \ldots & \text { (heuristic 8) }
\end{array}\right.
$$

3.2.2. Manufacturing Strategies. It is assumed that there are the following manufacturing strategies:

(i) The manufacturing process continues according to the chosen heuristic algorithm until each element $z_{\mu, \eta}^{k}$ chosen for production is made and only then the next determined element is subject to manufacturing; i.e., it is allowed to enter the manufacturing zone.

(ii) The continuity of the manufacturing process is guaranteed by using every possible machine so a decision about manufacturing is made immediately. The priority is to put charge material into the machine first. The robotic arm can make all other transportation tasks after delivering the charge material to all determined machines. Only then can semiproducts be transported to the subsequent determined machines. 
Let us introduce the matrix of order priority (76):

$$
\Theta^{0}=\left[\theta_{m, n}^{0}\right]
$$

where

$\theta_{m, n}^{0}$ is the priority of the manufacturing task for the $m$-th customer who ordered the $n$-th product the stage $k=0$.

The priority element takes the following values: $\theta_{m, n}^{0}=1$ for the high priority and $\theta_{m, n}^{0}=0$ otherwise.

3.2.3. Control Approaches. It is assumed that there are the following control approaches to be discussed:

(i) The first method assumes that the sequence of manufactured orders remains unchanged throughout the whole manufacturing process, i.e., the manufacturing sequence is determined. An element of the order matrix to be made is chosen with the use of heuristic algorithms. In this case the system is treated as the serial manufacturing system and a need to implement the matrix of routes arises.

(ii) The second method is based on the principle that any element of the order matrix can be made without the need to follow any sequence restriction of manufacturing operations (the sequence is optional), i.e., a machine is chosen according to a specified heuristic algorithm in order to meet defined criteria.

(iii) The third method mixes two previous methods as some order matrix elements require a defined manufacturing sequence whereas others do not.

In case of the first approach the sequence of operations on the $n$-th product is predefined and the order of making products cannot be alternated. Searching for solutions minimising the total order making time consists in minimising the standstill time of the manufacturing system as a whole or its individual elements (machines). Moreover, the manufacturing time cannot be increased so there is a need to search for other solutions such as, e.g., minimising the total manufacturing time, minimising the replacement time of tools, minimising lost flow capacity of tools. It is assumed that exactly at the moment when a certain $n$-th order frees the manufacturing space another order element enters the manufacturing zone.

The modelled system requires introducing manufacturing criteria according to which the manufacturing procedures are controlled as follows:

(1) In order to minimise the total manufacturing time the time criterion of the production process is formulated (77):

$$
Q_{p r} \longrightarrow \min
$$

(2) In order to minimise the total replacement time the criterion of the replacement process is formulated (78):

$$
Q_{\text {repl }} \longrightarrow \min
$$

(3) In order to minimise the total replacement time the criterion of lost flow capacity of tools is formulated (79):

$$
Q_{l f c} \longrightarrow \min
$$

The losses during the manufacturing process influence the costs which increase then.

\section{Results and Discussion}

To validate the above iteration approach to creating the dedicated software and the subsequent simulation process certain sample data and assumption are taken into account for the purpose of the calculation procedure. The main goals of the case study include

(i) comparing the results of simulations for strategies and available heuristics;

(ii) analysing timescales of making order for chosen strategies: Q_pr_ii_at random, Q_repl_ii_at random, $Q_{-}$ lf﹎.ii_at random, Q_pr_ii_heuristic_2, Q_pr_i heuristic 1 ;

(iii) analysing the problem of the system elasticity depending on the system state and the system usage;

(iv) analysing results of the manufacturing process simulation for 5000 simulations;

(v) presenting and analysing the histogram of the multisimulation process for 5000 repetitions for the initial order for the strategy generating the minimal manufacturing time;

(vi) analysing the parallel and subsequent simulation results;

(vii) searching for the best solution of manufacturing and prolonged manufacturing costs;

(viii) the comparative cost analysis of the five discussed approaches.

The case study analysis requires assuming the following data:

$$
\begin{aligned}
d_{1}^{m, n} & \longrightarrow d_{2}^{m, n} \longrightarrow d_{3}^{m, n} \longrightarrow d_{4}^{m, n} \longrightarrow d_{5}^{m, n} ; \\
\sum_{k=1}^{K} \sum_{n=1}^{N} \sum_{m=1}^{M} \theta_{m, n}^{k} & =0 ; \\
\Xi & =\left[\begin{array}{lllll}
1 & 1 & 1 & -1 & -1
\end{array}\right] ; \\
A & =B ; \\
\alpha & =1
\end{aligned}
$$

The sample data necessary to carry out the simulation of the manufacturing are given as follows:

(i) manufacturing routes (Table 1);

(ii) manufacturing times (Table 2);

(iii) unit manufacturing costs (Table 3 ); 
TABLE 1: The flow of products, manufacturing routes.

\begin{tabular}{|c|c|c|c|c|c|c|c|c|c|c|c|c|c|c|c|c|c|c|c|c|c|c|c|c|c|c|c|c|c|}
\hline \multirow{2}{*}{$\begin{array}{l}1, n \\
1,1\end{array}$} & \multicolumn{5}{|c|}{$d_{(1, n), j}$} & \multicolumn{2}{|l|}{$2, n$} & \multicolumn{3}{|c|}{$d_{(2, n), j}$} & \multicolumn{3}{|c|}{$3, n$} & \multicolumn{3}{|c|}{$d_{(3, n), j}$} & \multicolumn{3}{|c|}{$4, n$} & \multicolumn{3}{|c|}{$d_{(4, n), j}$} & \multicolumn{3}{|c|}{$5, n$} & \multicolumn{3}{|c|}{$d_{(5, n), j}$} & \multirow[b]{2}{*}{1} \\
\hline & 1 & 3 & 2 & 1 & 4 & 2,1 & 1 & 2 & 2 & 2 & 4 & 3,1 & 3 & 3 & 4 & 4 & 1 & 4,1 & 3 & 3 & 4 & 3 & 1 & 5,1 & 4 & 1 & 2 & 1 & \\
\hline 1,2 & 3 & 1 & 1 & 1 & 3 & 2,2 & 1 & 4 & 2 & 1 & 4 & 3,2 & 3 & 4 & 4 & 4 & 4 & 4,2 & 3 & 1 & 2 & 2 & 3 & 5,2 & 3 & 4 & 1 & 3 & 4 \\
\hline 1,3 & 3 & 1 & 4 & 1 & 2 & 2,3 & 2 & 1 & 4 & 1 & 1 & 3,3 & 2 & 4 & 3 & 1 & 2 & 4,3 & 2 & 1 & 2 & 2 & 2 & 5,3 & 4 & 4 & 4 & 4 & 4 \\
\hline 1,4 & 1 & 2 & 1 & 3 & 1 & 2,4 & 2 & 4 & 3 & 4 & 2 & 3,4 & 1 & 2 & 4 & 1 & 4 & 4,4 & 2 & 3 & 4 & 2 & 3 & 5,4 & 1 & 4 & 1 & 4 & 3 \\
\hline 1,5 & 4 & 1 & 4 & 1 & 3 & 2,5 & 4 & 2 & 2 & 2 & 1 & 3,5 & 1 & 3 & 4 & 2 & 1 & 4,5 & 2 & 1 & 3 & 4 & 2 & 5,5 & 4 & 3 & 1 & 3 & 2 \\
\hline
\end{tabular}

TABLE 2: Manufacturing times.

\begin{tabular}{|c|c|c|c|c|c|c|c|c|c|c|c|c|c|c|c|c|c|c|c|c|c|c|c|c|c|c|c|c|c|}
\hline \multirow{2}{*}{$\frac{1, n}{1,1}$} & \multicolumn{4}{|c|}{$\tau_{(1, n) /(i, j)}^{p r(1)}$} & \multicolumn{3}{|c|}{$2, \mathrm{n}$} & \multicolumn{3}{|c|}{$\tau_{(2, n) /(i, j)}^{p r(1)}$} & \multicolumn{3}{|c|}{$3, \mathrm{n}$} & \multicolumn{3}{|c|}{$\tau_{(3, n) /(i, j)}^{p r(1)}$} & \multicolumn{3}{|c|}{$4, \mathrm{n}$} & \multicolumn{3}{|c|}{$\tau_{(4, n) /(i, j)}^{p r(1)}$} & \multicolumn{3}{|c|}{$5, \mathrm{n}$} & \multicolumn{3}{|c|}{$\tau_{(5, n) /(i, j)}^{p r(1)}$} & \\
\hline & 9 & 6 & 9 & 8 & 9 & 2,1 & 8 & 7 & 8 & 9 & 7 & 3,1 & 6 & 6 & 6 & 9 & 9 & 4,1 & 8 & 6 & 9 & 7 & 6 & 5,1 & 7 & 8 & 9 & 8 & 8 \\
\hline 1,2 & 7 & 7 & 6 & 7 & 9 & 2,2 & 7 & 9 & 8 & 8 & 7 & 3,2 & 6 & 7 & 8 & 8 & 9 & 4,2 & 7 & 9 & 9 & 8 & 7 & 5,2 & 9 & 7 & 6 & 8 & 7 \\
\hline 1,3 & 9 & 9 & 9 & 7 & 9 & 2,3 & 8 & 8 & 9 & 6 & 7 & 3,3 & 8 & 8 & 9 & 9 & 7 & 4,3 & 6 & 6 & 8 & 7 & 6 & 5,3 & 8 & 6 & 7 & 8 & 8 \\
\hline 1,4 & 8 & 9 & 6 & 6 & 6 & 2,4 & 8 & 7 & 8 & 8 & 7 & 3,4 & 8 & 8 & 9 & 8 & 9 & 4,4 & 8 & 9 & 9 & 6 & 7 & 5,4 & 9 & 8 & 9 & 8 & 9 \\
\hline 1,5 & 9 & 8 & 6 & 9 & 6 & 2,5 & 6 & 8 & 8 & 9 & 7 & 3,5 & 8 & 6 & 8 & 9 & 8 & 4,5 & 8 & 9 & 8 & 7 & 8 & 5,5 & 9 & 6 & 7 & 9 & 8 \\
\hline
\end{tabular}

TABLE 3: Unit manufacturing costs.

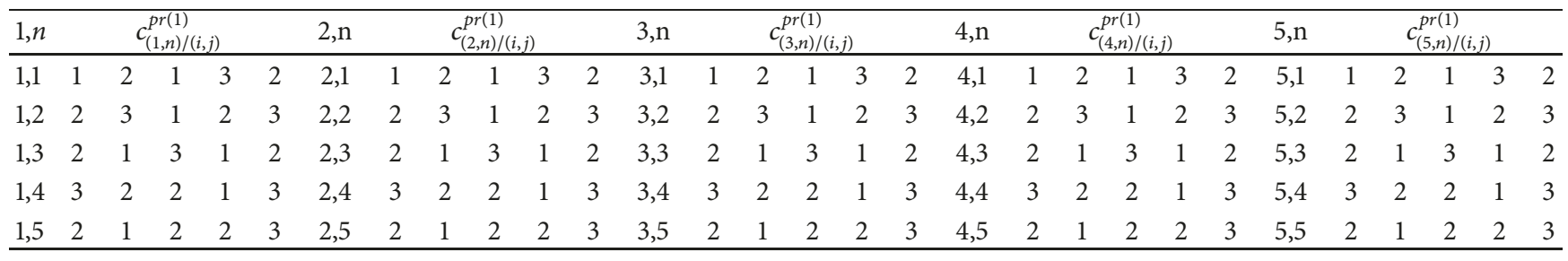

(iv) order matrix; order priority; coefficient of failures of tools; storing times and unit storing costs (Table 4);

(v) initial state of tools and life of tools (Table 5);

(vi) times and costs of assembling and disassembling of tools, transport of semiproducts to the key point and buffer zones, transport of charge, and transport of ready products (Table 6);

(vii) times and unit costs of replacement operations, transport of tools to and from machines for replacement; costs of maintenance, standstill, implementing heuristics, and predicted hidden costs (Table 7).

Sample data for the simulation process are not drawn from any external sources which would require special permission from any provider. They are defined by the authors with the sole purpose of demonstrating the functionality and usability of the mathematical model as well as the simulator created especially for the purpose of this paper. However, the sample data used by the authors in the paper partly result from observations of production processes taking place in the automotive manufacturing industry especially while making complex orders for identical products set by various customers.

There are two approaches to the problem of manufacturing. The first one requires waiting for the preceding order to leave the system, strategy i. Another one enables the operator of the system to start manufacturing immediately when such an opportunity emerges, strategy ii. First of all, the simulation process is carried out twice with the use of the implemented data for strategies i. and ii. The compared results of the simulation process are shown in Table 8.

It is visible that the winning strategy in terms of the total production time and effective production time is strategy ii. However, in terms of the robotic arm work as well as the total replacement time strategy $i$. is much more effective. The lost flow capacity is on par for both strategies nevertheless, it is worth noticing that heuristic 2 is the winning one in both approaches and the best of all in strategy $i$. The total transportation time is shorter in case of strategy i. where heuristic 2 is the best again. The final flow capacity is identical for all implemented heuristics other than for heuristic 2 which is better for strategy $\mathrm{i}$. The system flexibility indicator is the best again for heuristic 2; however, it is the best for strategy ii. using heuristic 2 .

Time scaling for Q_pr_ii_heuristic_2 is shown in Figure 2 and for Q_pr_i_heuristic_1 in Figure 3 and Q_pr_ii_at random in Figure 4 .

Figure 2 presents making order $Z^{0}$ for Q_pr_ii_heuristic_2 which enables parallel manufacturing. This approach eliminates standstill of machines and however, as seen in Table 14, generates extra costs due to parallel manufacturing tasks. The time scale shows that in the early manufacturing stages orders do not cumulate and only two of them are made at the same time. With the pass of manufacturing orders accumulate in the production system which leads to intensifying manufacturing of even four elements of the order matrix at the same time. 
TABLE 4: Order matrix; order priority; coefficient of failures of tools; storing times; and unit storing costs.

(a)

\begin{tabular}{lccccc}
\hline$z_{m, n}^{0}$ & $n=1$ & $n=2$ & $n=3$ & $n=4$ & $n=5$ \\
\hline$m=1$ & 29 & 24 & 26 & 27 & 7 \\
$m=2$ & 9 & 25 & 10 & 19 & 10 \\
$m=3$ & 23 & 24 & 28 & 9 & 27 \\
$m=4$ & 7 & 16 & 6 & 12 & 14 \\
$m=5$ & 31 & 12 & 14 & 16 \\
\hline
\end{tabular}

(b)

\begin{tabular}{|c|c|c|c|c|c|}
\hline$\overline{\theta_{m, n}^{k}}$ & $n=1$ & $n=2$ & $n=3$ & $n=4$ & $n=5$ \\
\hline$m=1$ & 0 & 0 & 0 & 0 & 0 \\
\hline$m=2$ & 0 & 0 & 0 & 0 & 0 \\
\hline$m=3$ & 0 & 0 & 0 & 0 & 0 \\
\hline$m=4$ & 0 & 0 & 0 & 0 & 0 \\
\hline$m=5$ & 0 & 0 & 0 & 0 & 0 \\
\hline
\end{tabular}

(c)

\begin{tabular}{lccccc}
\hline$\gamma_{m, n}^{\text {fail }(1)}$ & $n=1$ & $n=2$ & $n=3$ & $n=4$ & 9 \\
\hline$m=1$ & 5 & 6 & 3 & 5 & 9 \\
$m=2$ & 6 & 5 & 7 & 1 & 4 \\
$m=3$ & 5 & 5 & 7 & 7 & 1 \\
$m=4$ & 5 & 6 & 7 & 5 & 4 \\
$m=5$ & 6 & 8 & 3 & 4 \\
\hline
\end{tabular}

(d)

\begin{tabular}{lccccc}
\hline$c_{m, n}^{\text {fail }(1)}$ & $n=1$ & $n=2$ & $n=3$ & $n=4$ & 8 \\
\hline$m=1$ & 7 & 8 & 8 & 7 & 8 \\
$m=2$ & 8 & 7 & 8 & 7 & 8 \\
$m=3$ & 8 & 7 & 8 & 8 & 8 \\
$m=4$ & 7 & 8 & 7 & 8 & 8 \\
$m=5$ & 8 & 7 & 8 & 8 \\
\hline
\end{tabular}

(e)

\begin{tabular}{lccccc}
\hline$\tau_{w /(m, n)}^{(1) s t}$ & $n=1$ & $n=2$ & $n=3$ & $n=4$ & 15 \\
\hline$m=1$ & 13 & 12 & 19 & 23 & 23 \\
$m=2$ & 25 & 17 & 14 & 31 \\
$m=3$ & 24 & 9 & 18 & 21 \\
$m=4$ & 19 & 29 & 31 & 7 & 17 \\
$m=5$ & 15 & 8 & 17 & 30 \\
\hline
\end{tabular}

(f)

\begin{tabular}{|c|c|c|c|c|c|}
\hline $\begin{array}{l}c_{w /(m, n)}^{(1) s t} \\
c^{\prime}\end{array}$ & $n=1$ & $n=2$ & $n=3$ & $n=4$ & $n=5$ \\
\hline$m=1$ & 4 & 5 & 5 & 5 & 4 \\
\hline$m=2$ & 5 & 4 & 4 & 5 & 5 \\
\hline$m=3$ & 5 & 5 & 5 & 4 & 4 \\
\hline$m=4$ & 4 & 5 & 4 & 5 & 5 \\
\hline$m=5$ & 5 & 4 & 5 & 5 & 4 \\
\hline
\end{tabular}

(g)

\begin{tabular}{lccccc}
\hline$\tau_{m, n}^{(1) \text { buf }_{\text {st }}}$ & $n=1$ & $n=2$ & $n=3$ & $n=4$ & 5 \\
\hline$m=1$ & 5 & 5 & 5 & 5 & 5 \\
$m=2$ & 5 & 5 & 5 & 5 & 5 \\
$m=3$ & 5 & 5 & 5 & 5 & 5 \\
$m=4$ & 5 & 5 & 5 & 5 & 5 \\
$m=5$ & 5 & 5 & 5 & 5 & 5 \\
\hline
\end{tabular}


(h)

\begin{tabular}{|c|c|c|c|c|c|}
\hline$c_{m, n}^{(1) b u f-s t}$ & $n=1$ & $n=2$ & $n=3$ & $n=4$ & $n=5$ \\
\hline$m=1$ & 1 & 2 & 1 & 1 & 1 \\
\hline$m=2$ & 1 & 2 & 1 & 2 & 2 \\
\hline$m=3$ & 2 & 1 & 2 & 2 & 1 \\
\hline$m=4$ & 1 & 2 & 1 & 2 & 2 \\
\hline$m=5$ & 1 & 4 & 2 & 5 & 1 \\
\hline
\end{tabular}

(i)

\begin{tabular}{lccccc}
\hline$\tau_{m, n}^{(1) t_{-} z}$ & $n=1$ & $n=2$ & $n=3$ & $n=4$ & 12 \\
\hline$m=1$ & 25 & 24 & 28 & 31 & 11 \\
$m=2$ & 14 & 17 & 13 & 26 & 73 \\
$m=3$ & 25 & 19 & 23 & 21 \\
$m=4$ & 31 & 18 & 14 & 19 & 14 \\
$m=5$ & 17 & 19 & 30 & 30 \\
\hline
\end{tabular}

(j)

\begin{tabular}{lccccc}
\hline$c_{m, n}^{(1)+z}$ & $n=1$ & $n=2$ & $n=3$ & $n=4$ & $n=5$ \\
\hline$m=1$ & 6 & 7 & 7 & 6 & 7 \\
$m=2$ & 7 & 6 & 6 & 6 & 5 \\
$m=3$ & 6 & 5 & 6 & 7 & 7 \\
$m=4$ & 7 & 6 & 6 & 5 & 6 \\
$m=5$ & 5 & 6 & 7 & 7 & 7 \\
\hline
\end{tabular}

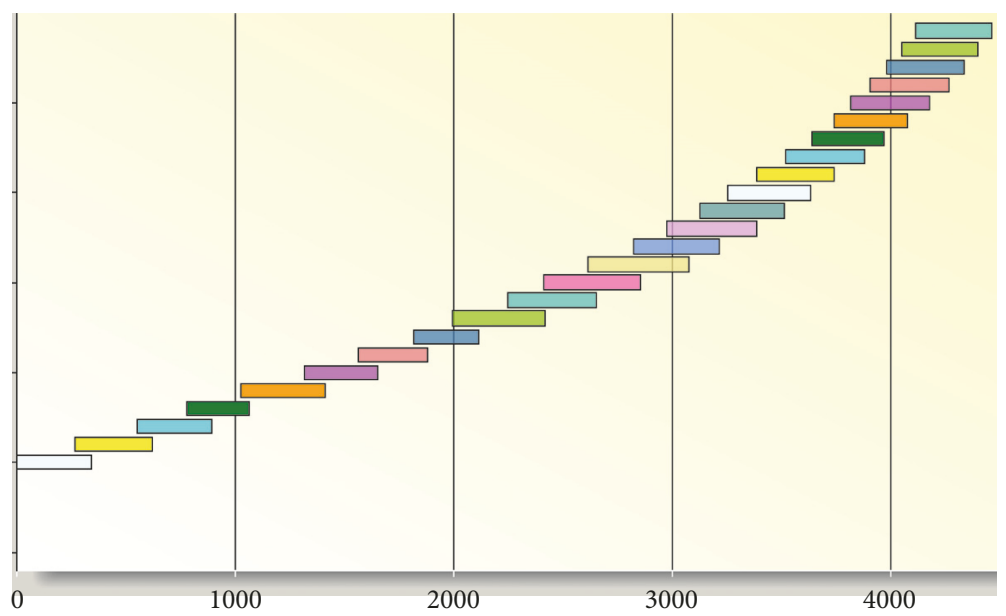

FIgURE 2: The timescale of making order $Z^{0}$ for Q_pr_ii_heuristic_2.

TABLE 5: Initial state of tools and life of tools.

(a)

\begin{tabular}{lccccc}
\hline$s_{n /(i, j)}^{(1 / 1)_{0}}$ & $j=1$ & $j=2$ & $j=3$ & $j=4$ & $j=5$ \\
\hline$i=1$ & 5 & 6 & 1 & 3 & 6 \\
$i=2$ & 2 & 6 & 3 & 0 & 2 \\
$i=3$ & 4 & 3 & 0 & 2 & 6 \\
$i=4$ & 2 & 2 & 2 & 1 & 1 \\
\hline
\end{tabular}

(b)

\begin{tabular}{lccccc}
\hline$g_{n /(i, j)}^{(1 / 1)}$ & $j=1$ & $j=2$ & $j=3$ & $j=4$ & $j=5$ \\
\hline$i=1$ & 6 & 8 & 7 & 8 & 9 \\
$i=2$ & 6 & 7 & 6 & 8 & 6 \\
$i=3$ & 6 & 6 & 7 & 9 & 9 \\
$i=4$ & 7 & 6 & 7 & 8 & 9 \\
\hline
\end{tabular}

Figure 3 presents making order $Z^{0}$ for Q_pr_i_heuristic_1 which excludes parallel manufacturing. The obvious thing emerging from this approach is the fact that the manufacturing process lasts much longer than in case of Q_pr_ii_heuristic_2; however, it does not generate excessive parallel manufacturing costs.

Figure 4 presents making order $Z^{0}$ for Q_pr_ii_at random which involves parallel manufacturing. The time scale results from carrying out 5000 simulation. The time scale characterised by the shortest order making time is presented in accordance with Table 14. Making orders is rather distributed evenly which results in making more than often at the same time in logistics system. Four times three orders are made at the same period of time. 
TABLE 6: Times and costs of assembling and disassembling of tools, transport of semiproducts to the key point and buffer zones, transport of charge, and transport of ready products.

\begin{tabular}{|c|c|c|c|c|c|c|c|c|c|c|}
\hline & $\tau_{(i, 1)}^{\text {assembl(1) }}$ & $\tau_{(i, 2)}^{\text {assembl }(1)}$ & $\tau_{(i, 3)}^{\text {assembl }(1)}$ & $\tau_{(i, 4)}^{\text {assembl }(1)}$ & $\tau_{(i, 5)}^{\text {assembl(1) }}$ & $c_{(i, 1)}^{\text {assembl }(1)}$ & $c_{(i, 2)}^{\text {assembl(1) }}$ & $c_{(i, 3)}^{\text {assembl }(1)}$ & $c_{(i, 4)}^{\text {assembl( } 1)}$ & $c_{(i, 5)}^{\text {assembl }(1)}$ \\
\hline$i=1$ & 6 & 9 & 7 & 8 & 7 & 5 & 4 & 3 & 5 & 3 \\
\hline$i=2$ & 6 & 8 & 9 & 9 & 7 & 3 & 4 & 4 & 5 & 3 \\
\hline$i=3$ & 7 & 7 & 8 & 7 & 6 & 3 & 4 & 3 & 4 & 4 \\
\hline \multirow{2}{*}{$i=4$} & 7 & 8 & 6 & 6 & 6 & 4 & 3 & 3 & 5 & 4 \\
\hline & $\tau_{(i, 1)}^{\text {disas }(1)}$ & $\tau_{(i, 2)}^{\operatorname{disas}(1)}$ & $\tau_{(i, 3)}^{\text {disas }(1)}$ & $\tau_{(i, 4)}^{\text {disas }(1)}$ & $\tau_{(i, 5)}^{\text {disas }(1)}$ & $c_{(i, 1)}^{\operatorname{disas}(1)}$ & $c_{(i, 2)}^{\text {disas }(1)}$ & $c_{(i, 3)}^{\text {disas }(1)}$ & $c_{(i, 4)}^{\text {disas }(1)}$ & $c_{(i, 5)}^{\text {disas }(1)}$ \\
\hline$i=1$ & 6 & 7 & 9 & 7 & 6 & 4 & 3 & 3 & 4 & 4 \\
\hline$i=2$ & 8 & 7 & 6 & 8 & 7 & 4 & 3 & 3 & 5 & 4 \\
\hline$i=3$ & 8 & 7 & 7 & 6 & 9 & 3 & 4 & 4 & 3 & 3 \\
\hline \multirow[t]{2}{*}{$i=4$} & 7 & 7 & 6 & 6 & 9 & 4 & 3 & 4 & 5 & 4 \\
\hline & $\tau_{(i, 1) / \longrightarrow .}^{\operatorname{tr}(1)}$ & $\tau_{(i, 2)\rfloor \longrightarrow .}^{\operatorname{tr}(1)}$ & $\tau_{(i, 3) / \longrightarrow .}^{\operatorname{tr}(1)}$ & $\tau_{(i, 4) / \longrightarrow}^{\operatorname{tr}(1)}$ & $\tau_{(i, 5) / \longrightarrow}^{\operatorname{tr}(1)}$ & $c_{(i, 1) / \longrightarrow .}^{\operatorname{tr}(1)}$ & $c_{(i, 2)\rfloor \longrightarrow .}^{\operatorname{tr}(1)}$ & $c_{(i, 3) / \longrightarrow .}^{\operatorname{tr}(1)}$ & $c_{(i, 4)\rfloor \longrightarrow .}^{\operatorname{tr}(1)}$ & $c_{(i, 5) / \longrightarrow .}^{\operatorname{tr}(1)}$ \\
\hline$i=1$ & 9 & 6 & 6 & 6 & 7 & 2 & 3 & 2 & 3 & 2 \\
\hline$i=2$ & 7 & 7 & 9 & 7 & 7 & 2 & 2 & 3 & 2 & 1 \\
\hline$i=3$ & 7 & 8 & 9 & 8 & 9 & 2 & 1 & 2 & 3 & 2 \\
\hline \multirow[t]{2}{*}{$i=4$} & 6 & 6 & 8 & 8 & 6 & 2 & 2 & 1 & 2 & 1 \\
\hline & $\tau_{\cdot \longrightarrow /(i, 2)}^{\operatorname{tr}(1)}$ & $\tau_{\cdot \longrightarrow /(i, 3)}^{\operatorname{tr}(1)}$ & $\tau_{(i, 4) / \bullet \longrightarrow}^{\operatorname{tr}(1)}$ & $\tau_{(i, 5) / \bullet \longrightarrow}^{\operatorname{tr}(1)}$ & $\tau_{\left(i, z_{s t}\right) / \cdot \longrightarrow}^{\operatorname{tr}(1)}$ & $c_{\bullet}^{\operatorname{tr}(1)}$ & $c_{\cdot}^{\operatorname{tr}(1)} \longrightarrow /(i, 3)$ & $c_{(i, 4) / \bullet}^{\operatorname{tr}(1)} \longrightarrow$ & $c_{(i, 5) / \bullet}^{\operatorname{tr}(1)} \longrightarrow$ & $c_{\left(i, z_{s t}\right) / \bullet \longrightarrow}^{\operatorname{trl}(1)}$ \\
\hline$i=1$ & 8 & 6 & 8 & 9 & 8 & 1 & 2 & 2 & 2 & 2 \\
\hline$i=2$ & 7 & 7 & 8 & 8 & 8 & 1 & 2 & 2 & 3 & 2 \\
\hline$i=3$ & 9 & 6 & 6 & 8 & 8 & 2 & 2 & 1 & 2 & 1 \\
\hline \multirow[t]{2}{*}{$i=4$} & 9 & 6 & 9 & 6 & 9 & 1 & 2 & 3 & 1 & 2 \\
\hline & $\tau_{(i, 1) \longrightarrow b_{1}}^{\operatorname{tr}(1)}$ & $\tau_{(i, 2) \longrightarrow b_{2}}^{\operatorname{tr}(1)}$ & $\tau_{(i, 3) \longrightarrow b_{3}}^{\operatorname{tr}(1)}$ & $\tau_{(i, 4) \longrightarrow b_{4}}^{\operatorname{tr}(4)}$ & $\tau_{(i, 5) \longrightarrow b_{5}}^{\operatorname{tr}(5)}$ & $c_{(i, 1) \longrightarrow b_{1}}^{\operatorname{tr}(1)}$ & $c_{(i, 2) \longrightarrow b_{2}}^{\operatorname{tr}(1)}$ & $c_{(i, 3) \longrightarrow b_{3}}^{\operatorname{tr}(1)}$ & $c_{(i, 4) \longrightarrow b_{4}}^{\operatorname{tr}(4)}$ & $c_{(i, 5) \longrightarrow b_{5}}^{\operatorname{tr}(5)}$ \\
\hline$i=1$ & 6 & 9 & 6 & 0 & 0 & 1 & 2 & 1 & 0 & 0 \\
\hline$i=2$ & 9 & 8 & 9 & 0 & 0 & 2 & 1 & 2 & 0 & 0 \\
\hline$i=3$ & 8 & 8 & 9 & 0 & 0 & 1 & 2 & 1 & 0 & 0 \\
\hline \multirow[t]{2}{*}{$i=4$} & 8 & 9 & 8 & 0 & 0 & 2 & 1 & 2 & 0 & 0 \\
\hline & $\tau_{b_{1} \longrightarrow(i, 2)}^{\operatorname{tr}(1)}$ & $\tau_{b_{2} \longrightarrow(i, 3)}^{\operatorname{tr}(1)}$ & $\tau_{b_{3} \longrightarrow(i, 4)}^{\operatorname{tr}(1)}$ & $\tau_{b_{4}}^{\operatorname{tr}(1)}$ & $\tau_{b_{5} \longrightarrow\left(i, z_{s t}\right)}^{\operatorname{tr}(1)}$ & $c_{b_{1}}^{\operatorname{tr}(1)}$ & $c_{b_{2} \longrightarrow(i, 3)}^{\operatorname{tr}(1)}$ & $c_{b_{3}}^{\operatorname{tr}(1)}(i, 4)$ & $c_{b_{4}}^{\operatorname{tr}(1)}(i, 5)$ & $c_{b_{5} \longrightarrow\left(i, z_{s t}\right)}^{\operatorname{tr}(1)}$ \\
\hline$i=1$ & 6 & 6 & 7 & 0 & 0 & 2 & 2 & 1 & 0 & 0 \\
\hline$i=2$ & 8 & 8 & 6 & 0 & 0 & 2 & 1 & 2 & 0 & 0 \\
\hline$i=3$ & 8 & 8 & 8 & 0 & 0 & 1 & 2 & 1 & 0 & 0 \\
\hline \multirow[t]{2}{*}{$i=4$} & 7 & 7 & 7 & 0 & 0 & 2 & 1 & 2 & 0 & 0 \\
\hline & $\begin{array}{c}\tau_{c h \longrightarrow(i, 1)}^{\operatorname{tr}(1)_{-}(m, n)} \\
\end{array}$ & $\begin{array}{c}\tau_{c h \longrightarrow(i, 2)}^{\operatorname{tr}(1)_{-}(m, n)} \\
\end{array}$ & $\begin{array}{c}\tau_{c h \longrightarrow(i, 3)}^{t r(1)_{-}(m, n)} \\
\end{array}$ & $\begin{array}{c}\tau_{c h}^{t r(1)_{-}(m, n)}(i, 4) \\
\end{array}$ & 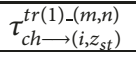 & $\begin{array}{l}c_{c h \longrightarrow(i, 1)}^{t r(1) \_(m, n)} \\
{ }_{c}\end{array}$ & $\begin{array}{c}c_{c h \longrightarrow(i, 2)}^{\operatorname{tr}(1)_{-}(m, n)} \\
{ }^{2}(i)\end{array}$ & 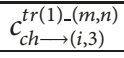 & $\begin{array}{c}c_{c h \longrightarrow(i, 4)}^{t r(1)_{-}(m, n)} \\
\end{array}$ & $\begin{array}{l}c_{c h}^{t r(1) \_(m, n)} \\
c_{\left(i, z_{s t}\right)}\end{array}$ \\
\hline$i=1$ & 9 & 0 & 0 & 0 & 0 & 2 & 0 & 0 & 0 & 0 \\
\hline$i=2$ & 6 & 0 & 0 & 0 & 0 & 2 & 0 & 0 & 0 & 0 \\
\hline$i=3$ & 9 & 0 & 0 & 0 & 0 & 2 & 0 & 0 & 0 & 0 \\
\hline \multirow[t]{2}{*}{$i=4$} & 6 & 0 & 0 & 0 & 0 & 2 & 0 & 0 & 0 & 0 \\
\hline & $\tau_{(i, 1) \longrightarrow z_{s t}}^{\operatorname{tr}(1) \_(m, n)}$ & $\tau_{(i, 2) \longrightarrow z_{s t}}^{\operatorname{tr}(1)(m, n)}$ & $\tau_{(i, 3) \longrightarrow z_{s t}}^{\operatorname{tr}(1)(m, n)}$ & $\tau_{(i, 4) \longrightarrow z_{s t}}^{\operatorname{tr}(1)_{(}(m, n)}$ & $\tau_{(i, 5) \longrightarrow z_{s t}}^{\operatorname{tr}(1)_{(}(m, n)}$ & $c_{(i, 1) \longrightarrow z_{s t}}^{\operatorname{tr}(1)_{(}(m, n)}$ & $c_{(i, 2) \longrightarrow z_{s t}}^{\operatorname{tr}(1) \_(m, n)}$ & $c_{(i, 3) \longrightarrow z_{s t}}^{t r(1)_{-}(m, n)}$ & $c_{(i, 4) \longrightarrow z_{s t}}^{\operatorname{tr}(1)(m, n)}$ & $c_{(i, 5) \longrightarrow z_{s t}}^{\operatorname{tr}(1)_{-}(m, n)}$ \\
\hline$i=1$ & 0 & 0 & 0 & 0 & 6 & 0 & 0 & 0 & 0 & 2 \\
\hline$i=2$ & 0 & 0 & 0 & 0 & 7 & 0 & 0 & 0 & 0 & 2 \\
\hline$i=3$ & 0 & 0 & 0 & 0 & 7 & 0 & 0 & 0 & 0 & 2 \\
\hline$i=4$ & 0 & 0 & 0 & 0 & 8 & 0 & 0 & 0 & 0 & 2 \\
\hline
\end{tabular}

Each simulation is also characterised by its elasticity which is the function of the system state and its usage. For the purpose of demonstration Figure 5 presents system elasticity for Q_pr_i_heuristic_1 whereas Figure 6 shows system elasticity for Q_pr_ii_heuristic_2.

As system elasticity is the function of system state and its usage it is visible how these two factors influence it. The system elasticity is high when its usage is high and the system imitates the lasting manufacturing process. At the same time the lasting manufacturing process leads to increasing its state values which as a result leads to the necessity of replacement worn out tool. As a consequence each replacement process decreases the state of the system enabling resuming the manufacturing procedures. After carrying out 5000 simulations of 
TABLE 7: Times and unit costs of replacement operations, transport of tools to and from machines for replacement; costs of maintenance, standstill, implementing heuristics, and predicted hidden costs.

\begin{tabular}{|c|c|c|c|c|c|c|c|c|c|c|}
\hline & $\tau_{i, 1}^{\alpha / 1}$ & $\tau_{i, 2}^{\alpha / 1}$ & $\tau_{i, 3}^{\alpha / 1}$ & $\tau_{i, 4}^{\alpha / 1}$ & $\tau_{i, 5}^{\alpha / 1}$ & $c_{i, 1}^{\alpha / 1}$ & $c_{i, 2}^{\alpha / 1}$ & $c_{i, 3}^{\alpha / 1}$ & $c_{i, 4}^{\alpha / 1}$ & $c_{i, 5}^{\alpha / 1}$ \\
\hline$i=1$ & 5 & 6 & 8 & 7 & 6 & 5 & 6 & 8 & 7 & 6 \\
\hline$i=2$ & 7 & 4 & 6 & 7 & 6 & 7 & 4 & 6 & 7 & 6 \\
\hline$i=3$ & 4 & 7 & 6 & 5 & 7 & 4 & 7 & 6 & 5 & 7 \\
\hline \multirow[t]{2}{*}{$i=4$} & 6 & 5 & 6 & 6 & 7 & 6 & 5 & 6 & 6 & 7 \\
\hline & $\tau_{i, 1}^{\alpha_{i n} / 1}$ & $\tau_{i, 2}^{\alpha_{i n} / 1}$ & $\tau_{i, 3}^{\alpha_{i n} / 1}$ & $\tau_{i, 4}^{\alpha_{i n} / 1}$ & $\tau_{i, 5}^{\alpha_{\text {in }} / 1}$ & $c_{i, 1}^{\alpha_{i n} / 1}$ & $c_{i, 2}^{\alpha_{i n} / 1}$ & $c_{i, 3}^{\alpha_{i n} / 1}$ & $c_{i, 4}^{\alpha_{\text {in }} / 1}$ & $c_{i, 5}^{\alpha_{\text {in }} / 1}$ \\
\hline$i=1$ & 6 & 7 & 7 & 9 & 4 & 4 & 5 & 9 & 10 & 3 \\
\hline$i=2$ & 4 & 5 & 7 & 6 & 7 & 8 & 3 & 4 & 8 & 2 \\
\hline$i=3$ & 5 & 8 & 7 & 6 & 8 & 5 & 6 & 8 & 6 & 6 \\
\hline \multirow[t]{2}{*}{$i=4$} & 7 & 6 & 7 & 7 & 6 & 5 & 4 & 7 & 7 & 3 \\
\hline & $\tau_{i, 1}^{\alpha_{\text {out }} / 1}$ & $\tau_{i, 2}^{\alpha_{\text {out }} / 1}$ & $\tau_{i, 3}^{\alpha_{\text {out }} / 1}$ & $\tau_{i, 4}^{\alpha_{\text {out }} / 1}$ & $\tau_{i, 5}^{\alpha_{\text {out }} / 1}$ & $c_{i, 1}^{\alpha_{\text {out }} / 1}$ & $c_{i, 2}^{\alpha_{\text {out }} / 1}$ & $c_{i, 3}^{\alpha_{\text {out }} / 1}$ & $c_{i, 4}^{\alpha_{\text {out }} / 1}$ & $c_{i, 5}^{\alpha_{\text {out }} / 1}$ \\
\hline$i=1$ & 5 & 6 & 5 & 8 & 5 & 3 & 2 & 4 & 5 & 3 \\
\hline$i=2$ & 5 & 6 & 11 & 4 & 7 & 5 & 2 & 5 & 3 & 5 \\
\hline$i=3$ & 6 & 7 & 6 & 5 & 9 & 4 & 3 & 5 & 2 & 3 \\
\hline \multirow[t]{2}{*}{$i=4$} & 6 & 7 & 8 & 4 & 3 & 4 & 5 & 7 & 6 & 4 \\
\hline & $c_{i, 1}^{(1) \text { main }}$ & $c_{i, 2}^{(1) \text { main }}$ & $c_{i, 3}^{(1) \text { main }}$ & $c_{i, 4}^{(1) \text { main }}$ & $c_{i, 5}^{(1) \text { main }}$ & $c_{(\mathrm{i}, 1) /(\mathrm{m}, \mathrm{n})}^{(1) \mathrm{still}}$ & $c_{(\mathrm{i}, 2) /(\mathrm{m}, \mathrm{n})}^{(1) \mathrm{still}}$ & $c_{(\mathrm{i}, 3) /(\mathrm{m}, \mathrm{n})}^{(1) \mathrm{still}}$ & $c_{(\mathrm{i}, 4) /(\mathrm{m}, \mathrm{n})}^{(1) \mathrm{still}}$ & $c_{(\mathrm{i}, 5) /(\mathrm{m}, \mathrm{n})}^{(1) \text { still }}$ \\
\hline$i=1$ & 9 & 11 & 13 & 9 & 10 & 3 & 4 & 4 & 4 & 3 \\
\hline$i=2$ & 12 & 14 & 9 & 15 & 14 & 4 & 4 & 3 & 4 & 4 \\
\hline$i=3$ & 13 & 18 & 9 & 12 & 13 & 3 & 3 & 4 & 4 & 4 \\
\hline \multirow[t]{2}{*}{$i=4$} & 11 & 19 & 12 & 20 & 9 & 4 & 4 & 3 & 3 & 3 \\
\hline & $\begin{array}{c}c_{(i, 1) /(m, n)}^{(1) \text { heur }} \\
c^{2}(m)\end{array}$ & $c_{(i, 2 /(m, n)}^{(1) \text { heur }}$ & $\begin{array}{c}c_{(i, 3) /(m, n)}^{(1) \text { heur }} \\
\end{array}$ & $\begin{array}{c}c_{(i, 4) /(m, n)}^{(1) \text { heur }} \\
c^{2}\end{array}$ & $\begin{array}{c}c_{(i, 5) /(m, n)}^{(1) \text { heur }} \\
\end{array}$ & $c_{(\mathrm{i}, 1) /(\mathrm{m}, \mathrm{n})}^{(1), h c}$ & $c_{(\mathrm{i}, 2) /(\mathrm{m}, \mathrm{n})}^{(1), h \mathrm{c}}$ & $c_{(\mathrm{i}, 3) /(\mathrm{m}, \mathrm{n})}^{(1), h c}$ & $c_{(\mathrm{i}, 4) /(\mathrm{m}, \mathrm{n})}^{(1) h c}$ & 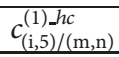 \\
\hline$i=1$ & 3 & 2 & 3 & 3 & 3 & 45 & 39 & 34 & 48 & 64 \\
\hline$i=2$ & 2 & 3 & 2 & 2 & 3 & 34 & 29 & 42 & 46 & 37 \\
\hline$i=3$ & 3 & 2 & 3 & 3 & 2 & 30 & 37 & 34 & 37 & 36 \\
\hline$i=4$ & 2 & 3 & 3 & 3 & 3 & 46 & 35 & 47 & 40 & 20 \\
\hline
\end{tabular}

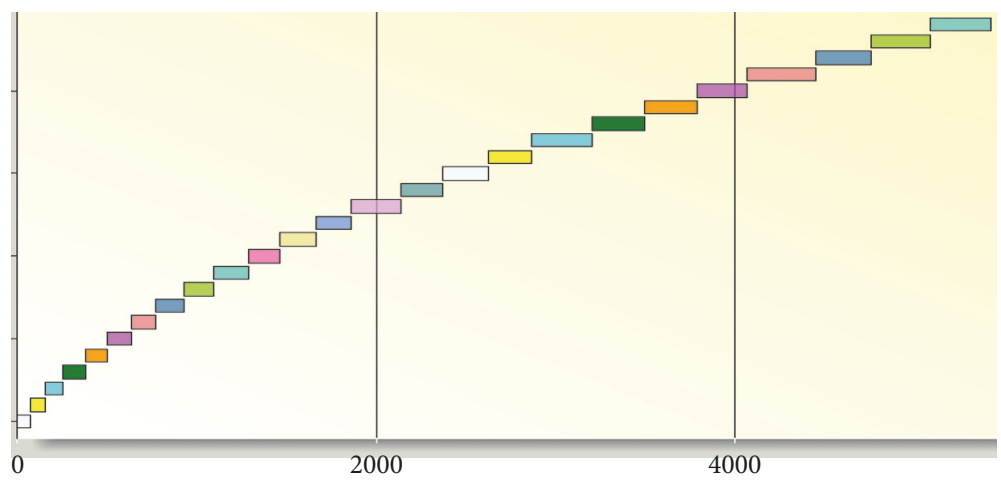

Figure 3: The timescale of making order $Z^{0}$ for Q_pr_i_heuristic_1.

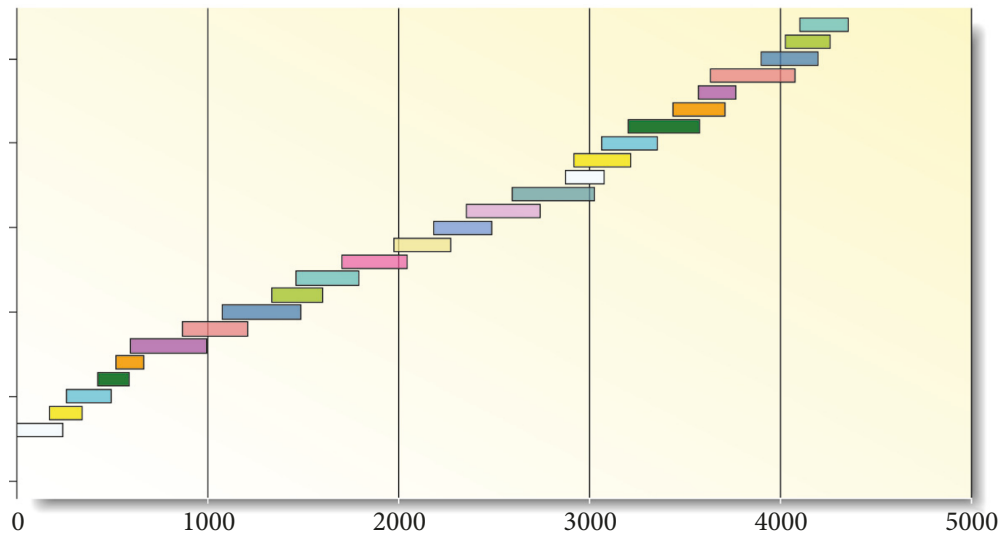

FIGURE 4: Time scaling of parallel manufacturing of the order $Z^{0}$ for Q_pr_ii_at random. 


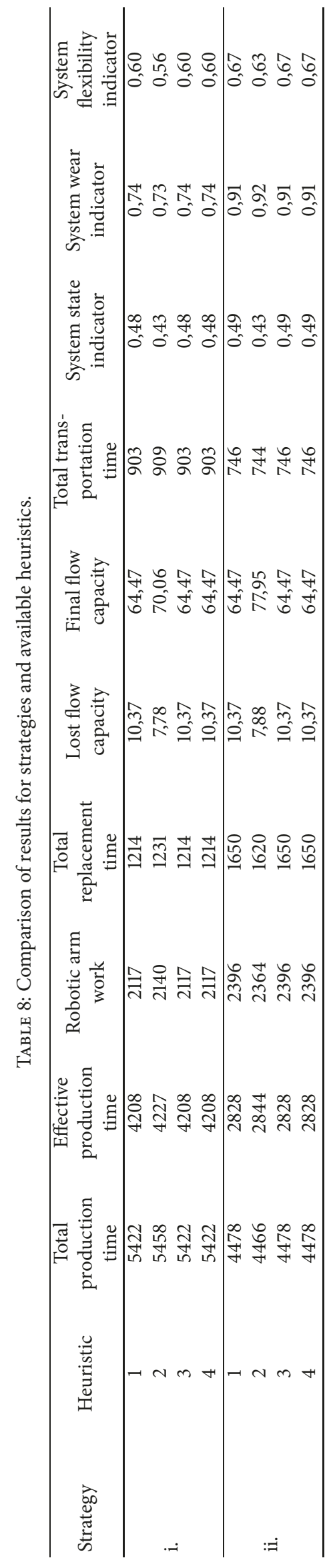




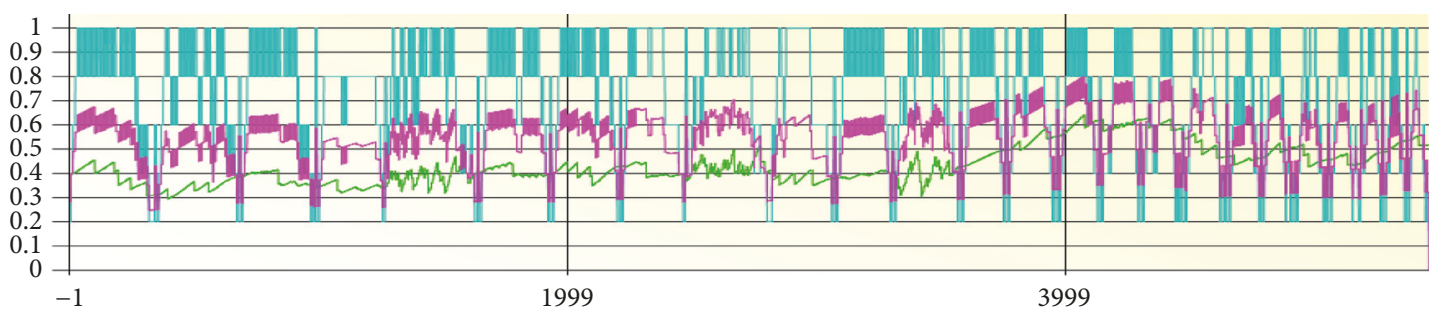

System state
_ System usage
System elasticity

FIGURE 5: System elasticity for Q_pr_i_heuristic_1.

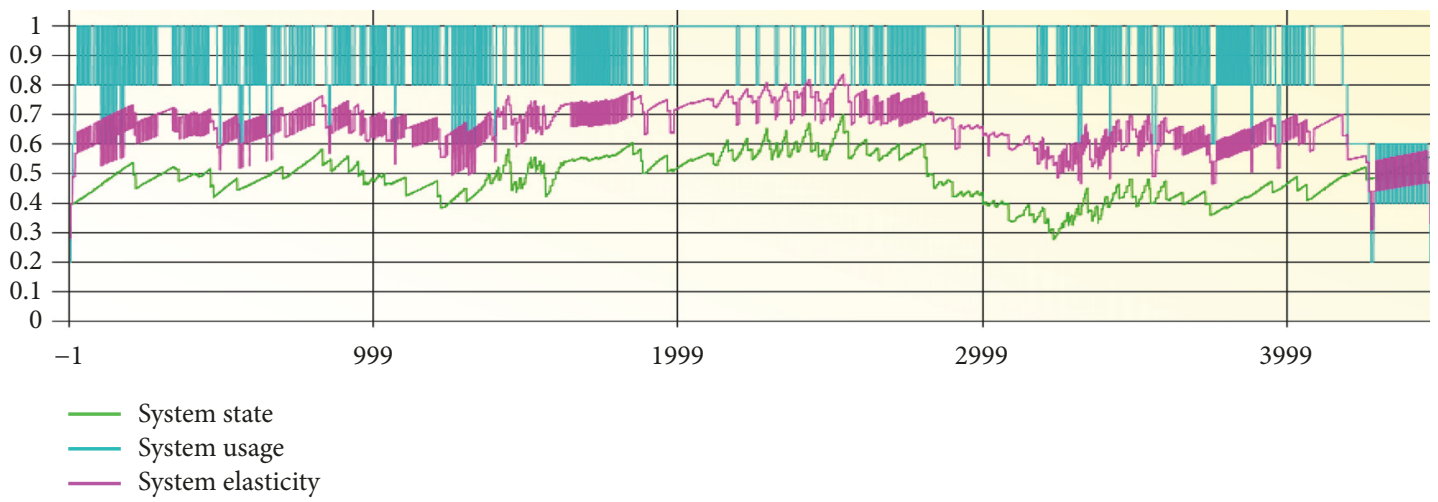

FIGURE 6: System elasticity for Q_pr_ii_heuristic_2.

TABLE 9: Results of the manufacturing process simulation for 5000 simulations.

\begin{tabular}{lcccc}
\hline Simulation no. & Production time & Replacement time of tools & Lost flow capacity & Final flow capacity \\
\hline 2737 & 4356 & 1573 & 7,89 & 63,95 \\
1523 & 4393 & 1384 & 6,68 & 72,15 \\
1547 & 4432 & 1470 & 10,00 & 64,83 \\
4428 & 4445 & 1623 & 7,98 & 106,85 \\
\hline
\end{tabular}

the manufacturing process for Q_pr_ii_at random the results are arranged presented from the point of view of the criterion $Q_{p r} \longrightarrow$ min in Table 9 .

As seen in Table 9 the best total manufacturing time $T_{2737}^{p r}=4356$ (expressed in time units) is obtained in the simulation process no. 2737. However, this simulation procedure does not guarantee the minimal replacement time of tools which is much worse than the minimal replacement time of tools $T_{1523}^{r e p l}=1384$ obtained in the simulation process no. 1523. This difference which equals 189 time units is quite immense and may lead to financial losses; therefore, it does make sense to consider another kind of approach presented in the paper hereby. Moreover, the simulation process no. 1523 offers the minimal value of the lost flow capacity. Table 10 presents more detailed results from the point of view of $Q_{p r} \longrightarrow \min$. The results are segregated increasing according to the stated criterion where the minimal manufacturing time is searched for.
As seen in Table 11 the most minimal manufacturing time does not guarantee either the minimal tools replacement time or the lost flow capacity or the final flow capacity. Simulation no. 2737 out of the set of 5000 simulations delivered the satisfactory solution satisfying $Q_{p r} \longrightarrow \min$. We can assume that the probability of finding a better result in terms of $Q_{p r} \longrightarrow$ min is extremely low; however, it is not excluded.

Figure 7 presents results of 5000 multisimulation procedures in which 232 different results were obtained. The shortest manufacturing time equals 4356 time units whereas the longest manufacturing time equals 4672 time units. The spread equals 316 time units. The most repeated result equals 4464 time units and was obtained 64 times which is $1,3 \%$ of all simulation results.

Table 12 presents assumed time unit cost depending on the parallel manufacturing process in case of strategy ii. For example, if manufacturing of the order $z_{2,1}^{k}$ was ongoing and at one moment of this process manufacturing of the order 


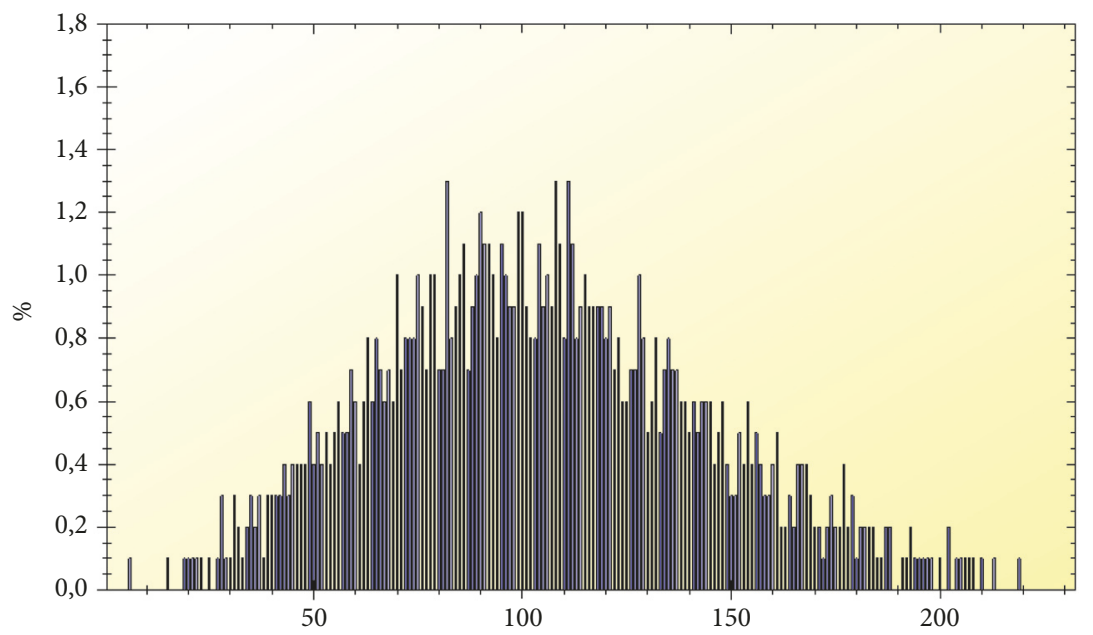

FIGURE 7: The histogram of the multisimulation process for 5000 repetitions for order $Z^{0}$ for strategy ii. with choosing heuristics at random.

TABLE 10: Results of the manufacturing process simulation for $Q_{p r} \longrightarrow$ min.

\begin{tabular}{lcccc}
\hline Simulation & Total production time & Tools replacement time & Lost flow capacity & Final flow capacity \\
\hline 2737 & 4356 & 1573 & 7,89 & 63,95 \\
527 & 4367 & 1536 & 7,07 & 68,77 \\
2082 & 4373 & 1542 & 6,71 & 78,13 \\
4623 & 4377 & 1502 & 8,23 & 74,61 \\
4854 & 4384 & 1632 & 7,01 & 80,83 \\
239 & 4384 & 1586 & 4,57 & 91,27 \\
1182 & 4386 & 1537 & 6,65 & 88,18 \\
4387 & 4386 & 1535 & 5,85 & 66,98 \\
2643 & 4386 & 1514 & 8,12 & 82,72 \\
1912 & 4387 & 1554 & 8,16 & 74,68 \\
4395 & 4387 & 1558 & 8,97 & 72,86 \\
\end{tabular}

TABLE 11: Results of the combined manufacturing simulation process for $Q_{p r} \longrightarrow \min , Q_{r e p l} \longrightarrow$ min, and $Q_{l f c} \longrightarrow$ min in case of 5000 simulation procedures.

\begin{tabular}{lccc}
\hline & $Q_{p r} \longrightarrow \min$ & $Q_{\text {repl }} \longrightarrow \min$ & $Q_{l f c} \longrightarrow \min$ \\
\hline Simulation no. & 2737 & 1523 & 2271 \\
Total manufacturing time & 4356 & 4393 & 1384 \\
Total replacement time & 1573 & 6,68 & 4504 \\
Lost flow capacity & 7,89 & 732 & 2,39 \\
Total transportation time & 726 & 0,50 & 750 \\
System state indicator & 0,44 & 0,92 & 0,47 \\
System wear indicator & 0,93 & 0,68 & 0,91 \\
System flexibility indicator & 0,64 & 72,2 & 0,65 \\
Final flow capacity & 64 & \multicolumn{2}{c}{} \\
\hline
\end{tabular}

$z_{1,4}^{k}$ began in a parallel way it is assumed that $c_{\mathrm{Q}_{-} r_{-}(2,1) /(1,4)}^{\text {par(1) }}=$ 5 . Nevertheless, as the company should be treated as one complex logistics system it was decided to calculate its uniform parallel manufacturing cost which in fact is its average parallel manufacturing cost, i.e., $c_{\mathrm{Q}_{-} \text {pr_av }}^{\text {par }(1)}=3,52$
Table 13 presents simulation results of parallel manufacturing for each order matrix element for Q_pr_ii_at random, Q_repl_ii_at random, and $Q_{-} l f_{-}$ii__at random, where the best results satisfying the stated criteria are chosen, as well as for Q_pr_ii_heuristic_2 in terms of parallel manufacturing and simulation results of the manufacturing process for 


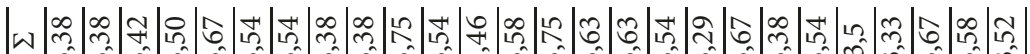

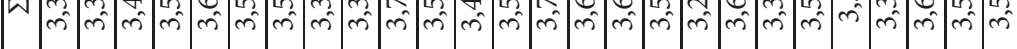

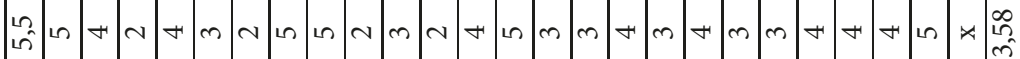

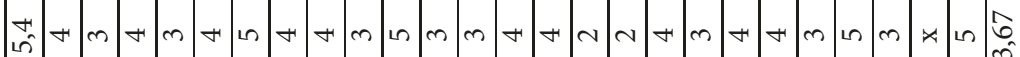

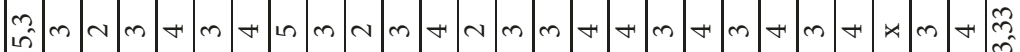

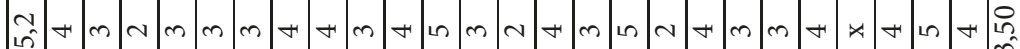

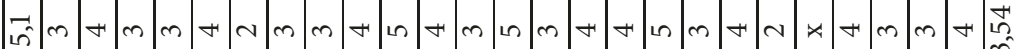

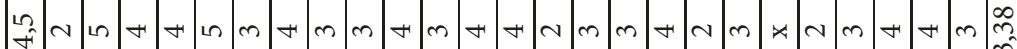

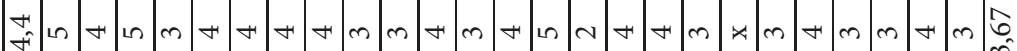

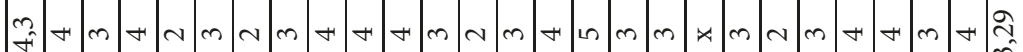

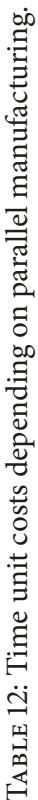

(1)

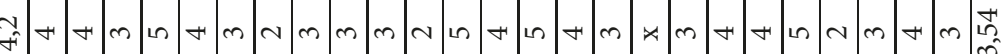

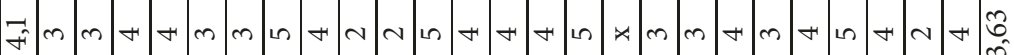

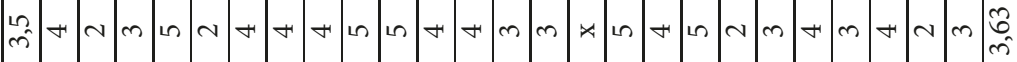

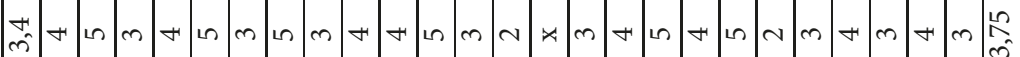

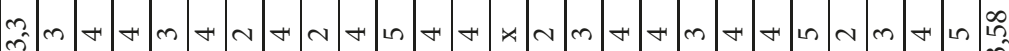

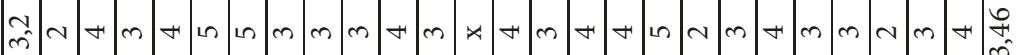

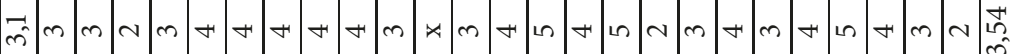

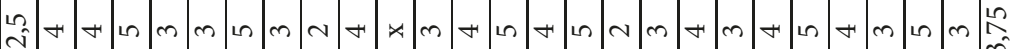

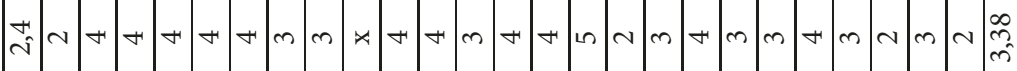

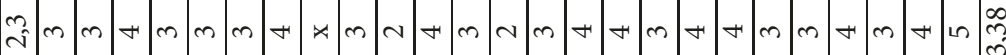

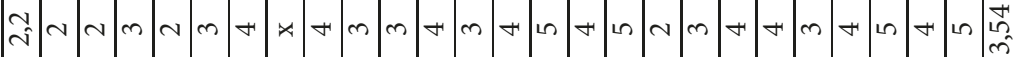

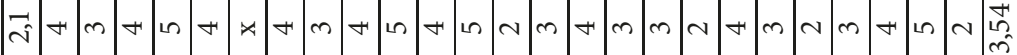

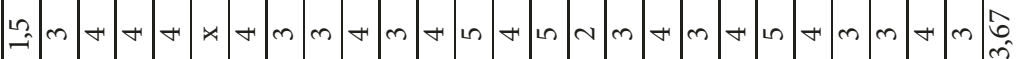

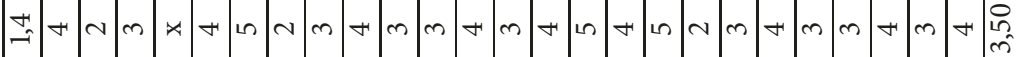

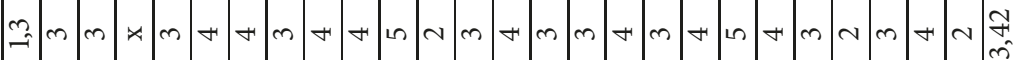

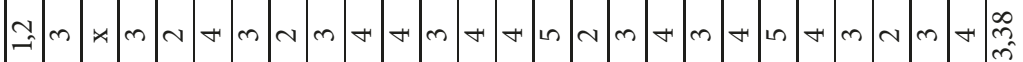

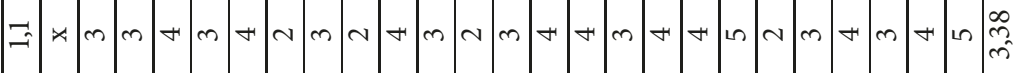

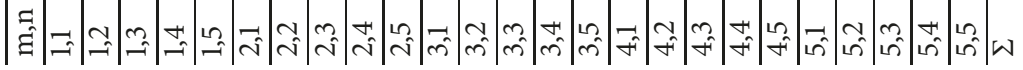


TABLE 13: Parallel and subsequent simulation results - the beginning and finishing moments of the manufacturing process.

\begin{tabular}{|c|c|c|c|c|c|c|c|c|c|c|c|c|}
\hline \multirow{2}{*}{$m, n$} & \multirow{2}{*}{$z_{m, n}^{0}$} & \multirow{2}{*}{$c_{m, n}^{p r-u}$} & \multicolumn{2}{|c|}{$\tau_{Q_{\text {p } p r}}^{\operatorname{par}(1)}$} & \multicolumn{2}{|c|}{$\tau_{\text {Qrepl }}^{\text {par(1) }}$} & \multicolumn{2}{|c|}{$\tau_{\mathrm{Q} \perp f c}^{\operatorname{par}(1)}$} & \multicolumn{2}{|c|}{$\tau_{i i j h c_{2} \text { par(1) }}^{\text {part }}$} & \multicolumn{2}{|c|}{$\tau_{i \jmath_{h c_{-} 1}^{\operatorname{man}(1)}}$} \\
\hline & & & Start & End & Start & End & Start & End & Start & End & Start & End \\
\hline 1,1 & 29 & 82 & 2595 & 3028 & 972 & 1362 & 3706 & 4305 & 268 & 618 & 4760 & 5090 \\
\hline 1,2 & 24 & 82 & 0 & 243 & 0 & 243 & 2042 & 2446 & 1815 & 2117 & 2624 & 2867 \\
\hline 1,3 & 26 & 79 & 1701 & 2044 & 168 & 486 & 1720 & 2120 & 1318 & 1650 & 3496 & 3788 \\
\hline 1,4 & 27 & 78 & 1460 & 1789 & 1254 & 1533 & 1480 & 1860 & 782 & 1061 & 3788 & 4067 \\
\hline 1,5 & 22 & 74 & 869 & 1209 & 766 & 1078 & 3182 & 3594 & 2412 & 2855 & 2136 & 2370 \\
\hline 2,1 & 9 & 71 & 426 & 586 & 3257 & 3574 & 1962 & 2221 & 3821 & 4182 & 257 & 384 \\
\hline 2,2 & 25 & 86 & 3202 & 3576 & 3642 & 4199 & 2218 & 2661 & 1567 & 1879 & 3204 & 3496 \\
\hline 2,3 & 10 & 71 & 4104 & 4356 & 678 & 874 & 2825 & 3129 & 3640 & 3971 & 503 & 633 \\
\hline 2,4 & 7 & 83 & 4026 & 4262 & 3073 & 3365 & 254 & 404 & 3986 & 4335 & 73 & 159 \\
\hline 2,5 & 10 & 63 & 168 & 340 & 1647 & 1807 & 3601 & 4001 & 3742 & 4077 & 633 & 773 \\
\hline 3,1 & 23 & 69 & 2183 & 2487 & 2918 & 3303 & 3432 & 3905 & 2250 & 2651 & 2370 & 2624 \\
\hline 3,2 & 24 & 84 & 1074 & 1489 & 3419 & 3958 & 0 & 340 & 1994 & 2422 & 2867 & 3204 \\
\hline 3,3 & 28 & 74 & 2353 & 2744 & 2421 & 2840 & 970 & 1387 & 550 & 892 & 4453 & 4760 \\
\hline 3,4 & 19 & 93 & 1973 & 2271 & 2015 & 2384 & 318 & 648 & 2618 & 3079 & 1861 & 2136 \\
\hline 3,5 & 27 & 80 & 597 & 993 & 1744 & 2140 & 528 & 990 & 1025 & 1409 & 4067 & 4453 \\
\hline 4,1 & 7 & 62 & 3571 & 3770 & 2217 & 2443 & 3988 & 4363 & 4051 & 4403 & 159 & 257 \\
\hline 4,2 & 16 & 87 & 2922 & 3220 & 3894 & 4343 & 2576 & 2946 & 2827 & 3220 & 1462 & 1663 \\
\hline 4,3 & 6 & 61 & 2878 & 3078 & 4045 & 4393 & 3388 & 3674 & 4115 & 4466 & 0 & 73 \\
\hline 4,4 & 9 & 87 & 517 & 665 & 3339 & 3663 & 2742 & 3031 & 3906 & 4270 & 384 & 503 \\
\hline 4,5 & 14 & 79 & 3438 & 3711 & 2281 & 2564 & 820 & 1123 & 3130 & 3514 & 1096 & 1286 \\
\hline 5,1 & 31 & 72 & 3636 & 4080 & 402 & 788 & 1212 & 1693 & 0 & 343 & 5090 & 5422 \\
\hline 5,2 & 12 & 82 & 1337 & 1605 & 2659 & 2958 & 3066 & 3390 & 3388 & 3742 & 773 & 932 \\
\hline 5,3 & 14 & 67 & 3067 & 3356 & 3137 & 3486 & 4052 & 4504 & 3254 & 3635 & 1286 & 1462 \\
\hline 5,4 & 12 & 96 & 3899 & 4198 & 2790 & 3075 & 2456 & 2806 & 3519 & 3885 & 932 & 1096 \\
\hline 5,5 & 16 & 80 & 261 & 493 & 1494 & 1699 & 2914 & 3286 & 2976 & 3386 & 1663 & 1861 \\
\hline \multicolumn{2}{|c|}{ Earliest start: } & & 168 & & 168 & & 254 & & 268 & & 0 & \\
\hline \multicolumn{2}{|c|}{ Latest start: } & & 4104 & & 4045 & & 4052 & & 4115 & & 5090 & \\
\hline
\end{tabular}

Q_pr_i_heuristic_1. The beginning and finishing moments of the manufacturing process are given.

Table 14 presents the simulation results of parallel manufacturing for Q_pr_ii_at random, Q_repl_ii_at random, and Q_lf__ii_at random as well as for Q_pr_ii_heuristic_2. The first three results were obtained by means of carrying out 5000 simulations for every approach and choosing the best result satisfying each criterion. Both the parallel manufacturing time for each order and the cost of parallel manufacturing for each order are given. The costs are summed for every approach and their average value is calculated.

As seen in Table 14 the lowest parallel manufacturing cost is obtained by implementing Q_pr_ii_at random whereas Q_lfc_ii_at random proves to be the most expensive when implemented.

Table 15 presents costs in case of prolonged manufacturing for sample data. The regular intervals of 10 costs units are taken into account for illustration reasons. The simulations were carried out for:

(1) Q_pr_ii_at random is the best result of 5000 simulations for the minimal manufacturing time criterion with the use of strategy ii. where heuristics are drawn at random.
(2) Q_repl_ii_at random is the best result of 5000 simulations for the minimal replacement time criterion with the use of strategy ii. where heuristics are drawn at random.

(3) Q_lfc_ii_at random is the best result of 5000 simulations for the minimal lost flow capacity criterion with the use of the strategy ii. where heuristics are drawn at random.

(4) Q_pr_ii_heuristic_2: strategy ii. is implemented for heuristic 2 .

(5) Q_pr_i_heuristic_1: strategy i. is implemented for heuristic 1.

As seen in Table 15 and subsequently in Figure 8 the minimal prolonged manufacturing costs are generated by Q_pr_i_heuristic_1 which does not satisfy the minimal manufacturing time criterion. These costs equal the costs generated in case of implementing Q_pr_ii_at random when $c_{\text {prol }}=$ 90989,09307692 (see Table 16) and then start growing when the unit manufacturing cost increases making the manufacturing process unprofitable if this strategy is unchanged.

Figure 8 is the graphic representation of prolonged manufacturing. Each of the five approaches is described by its 
TABLE 14: Costs of parallel manufacturing.

\begin{tabular}{|c|c|c|c|c|c|c|c|c|c|}
\hline$m, n$ & $z_{m, n}^{0}$ & $\begin{array}{l}\tau_{\mathrm{Q}-p r}^{p a r}(\pi) \\
\end{array}$ & $c_{\mathrm{Q}-p r}^{\operatorname{par}(\pi)}$ & $\begin{array}{c}\tau_{\text {Q_repl }}^{\text {par }(\pi)} \\
\end{array}$ & $\begin{array}{c}c_{Q_{-} r e p l}^{\text {par }} \\
\end{array}$ & $\tau_{\mathrm{Q} \perp f \mathcal{c}}^{\operatorname{par}(\pi)}$ & $c_{\mathrm{Q} \perp f c}^{\operatorname{par}(\pi)}$ & $\begin{array}{l}\tau_{i i \_h c_{2} 2}^{p a r(\pi)} \\
\end{array}$ & 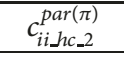 \\
\hline 1,1 & 29 & 150 & 528,00 & 108 & 380,16 & 317 & 1115,84 & 68 & 239,36 \\
\hline 1,2 & 24 & 75 & 264,00 & 75 & 264,00 & 228 & 802,56 & 123 & 432,96 \\
\hline 1,3 & 26 & 71 & 249,92 & 84 & 295,68 & 158 & 556,16 & 83 & 292,16 \\
\hline 1,4 & 27 & 88 & 309,76 & 39 & 137,28 & 140 & 492,80 & 36 & 126,72 \\
\hline 1,5 & 22 & 135 & 475,20 & 106 & 373,12 & 206 & 725,12 & 237 & 834,24 \\
\hline 2,1 & 9 & 69 & 242,88 & 235 & 827,20 & 179 & 630,08 & 276 & 971,52 \\
\hline 2,2 & 25 & 138 & 485,76 & 305 & 1073,60 & 205 & 721,60 & 64 & 225,28 \\
\hline 2,3 & 10 & 0 & 0,00 & 108 & 380,16 & 215 & 756,80 & 229 & 806,08 \\
\hline 2,4 & 7 & 158 & 556,16 & 228 & 802,56 & 86 & 302,72 & 284 & 999,68 \\
\hline 2,5 & 10 & 79 & 278,08 & 63 & 221,76 & 295 & 1038,40 & 256 & 901,12 \\
\hline 3,1 & 23 & 134 & 471,68 & 230 & 809,60 & 304 & 1070,08 & 239 & 841,28 \\
\hline 3,2 & 24 & 152 & 535,04 & 312 & 1098,24 & 86 & 302,72 & 172 & 605,44 \\
\hline 3,3 & 28 & 149 & 524,48 & 181 & 637,12 & 175 & 616,00 & 110 & 387,20 \\
\hline 3,4 & 19 & 88 & 309,76 & 167 & 587,84 & 120 & 422,40 & 252 & 887,04 \\
\hline 3,5 & 27 & 124 & 436,48 & 125 & 440,00 & 170 & 598,40 & 91 & 320,32 \\
\hline 4,1 & 7 & 134 & 471,68 & 162 & 570,24 & 311 & 1094,72 & 288 & 1013,76 \\
\hline 4,2 & 16 & 153 & 538,56 & 298 & 1048,96 & 204 & 718,08 & 244 & 858,88 \\
\hline 4,3 & 6 & 156 & 549,12 & 0 & 0,00 & 242 & 851,84 & 0 & 0,00 \\
\hline 4,4 & 9 & 68 & 239,36 & 244 & 858,88 & 206 & 725,12 & 284 & 999,68 \\
\hline 4,5 & 14 & 140 & 492,80 & 143 & 503,36 & 153 & 538,56 & 260 & 915,20 \\
\hline 5,1 & 31 & 181 & 637,12 & 110 & 387,20 & 213 & 749,76 & 75 & 264,00 \\
\hline 5,2 & 12 & 145 & 510,40 & 168 & 591,36 & 208 & 732,16 & 223 & 784,96 \\
\hline 5,3 & 14 & 154 & 542,08 & 229 & 806,08 & 0 & 0,00 & 247 & 869,44 \\
\hline 5,4 & 12 & 172 & 605,44 & 157 & 552,64 & 230 & 809,60 & 245 & 862,40 \\
\hline 5,5 & 16 & 67 & 235,84 & 52 & 183,04 & 220 & 774,40 & 256 & 901,12 \\
\hline \multicolumn{2}{|c|}{ Sum: } & 2980 & 10489,60 & 3929 & 13830,08 & 4871 & 17145,92 & 4642 & 16339,84 \\
\hline \multicolumn{2}{|c|}{ Average: } & 119,2 & 419,58 & 157,16 & 553,2032 & 194,84 & 685,8368 & 185,68 & 653,5936 \\
\hline
\end{tabular}

TABLE 15: Prolonged manufacturing costs for sample data.

\begin{tabular}{lccccc}
\hline$c_{\text {prol }}$ & Q_pr_ii_at random & Q_repl_ii_at random & Q_lfc_ii_at random & Q_pr_ii_heuristic_2 & Q_pr_i_heuristic_1 \\
\hline 10,00 & 90989,09 & 96400,77 & 110836,30 & 108291,36 & 79712,00 \\
15,00 & 90989,09 & 96585,77 & 111576,30 & 108841,36 & 85042,00 \\
20,00 & 90989,09 & 96770,77 & 112316,30 & 109391,36 & 90372,00 \\
25,00 & 90989,09 & 96955,77 & 113056,30 & 109941,36 & 95702,00 \\
30,00 & 90989,09 & 97140,77 & 113796,30 & 110491,36 & 101032,00 \\
35,00 & 90989,09 & 97325,77 & 114536,30 & 111041,36 & 106362,00 \\
40,00 & 90989,09 & 97510,77 & 115276,30 & 11591,36 & 111692,00 \\
45,00 & 90989,09 & 97695,77 & 116016,30 & 112141,36 & 117022,00 \\
50,00 & 90989,09 & 97880,77 & 116756,30 & 112691,36 & 122352,00 \\
55,00 & 90989,09 & 98065,77 & 117496,30 & 113241,36 & 127682,00 \\
60,00 & 90989,09 & 98250,77 & 118236,30 & 113791,36 & 133012,00 \\
\hline
\end{tabular}

function showing the dependence of the base manufacturing costs on the unit cost.

Manufacturing and prolonged manufacturing costs are included in Figure 8 . The vertical axis presents the values which are the sum of the base manufacturing costs and prolonged manufacturing costs. The horizontal axis presents the values adequate to the unit cost of the prolonged manufacturing. The obvious thing that emerges is the fact that Q_pr_i_heuristic_1 is profitable till the moment when the unit cost of the prolonged manufacturing reaches the value of 20,57888656 and the cost is on par with the costs of the manufacturing approach Q_pr_ii_at random. The sum of 


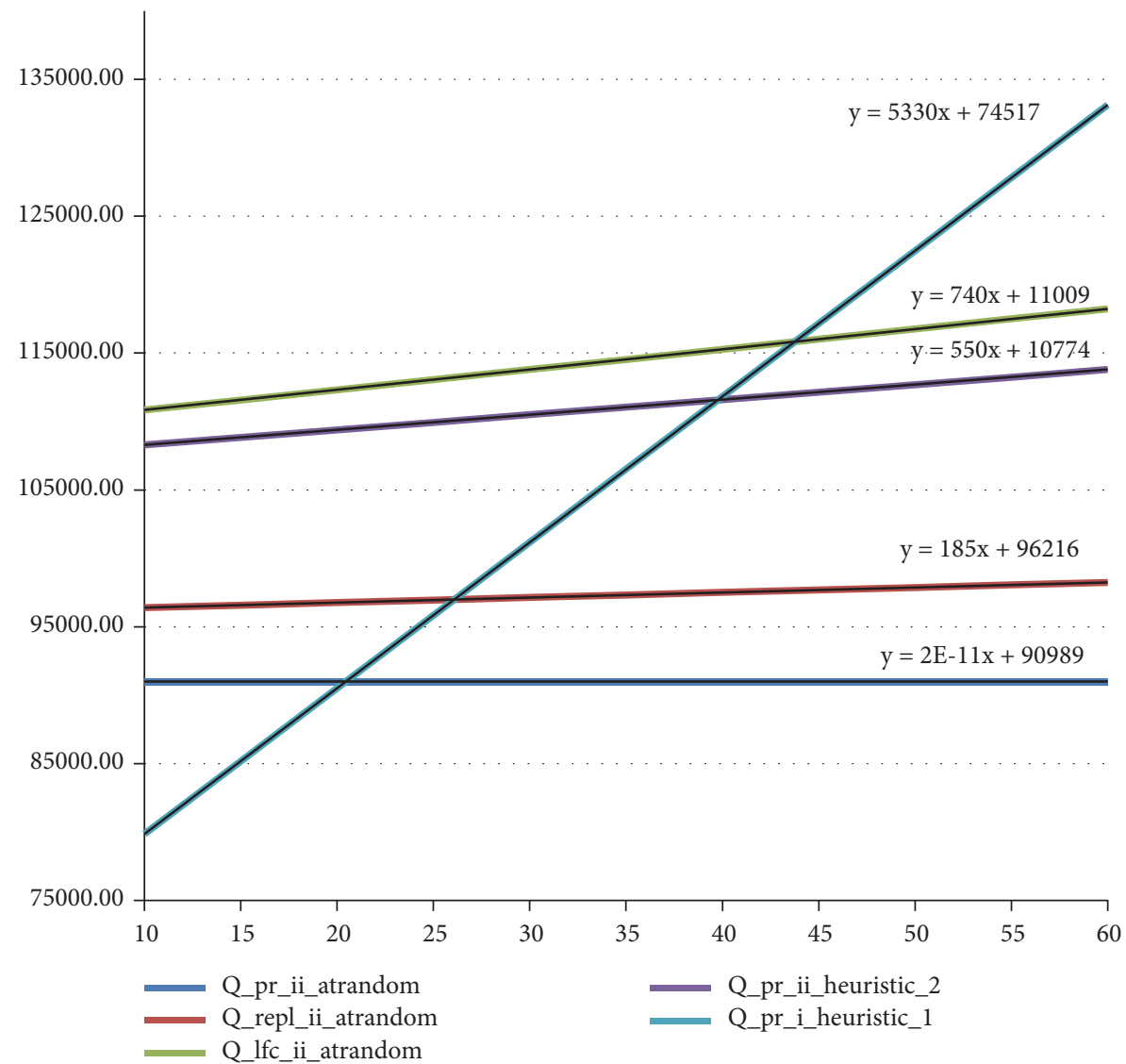

FIGURE 8: Prolonged manufacturing costs.

TABLE 16: Prolonged manufacturing costs, intersection points.

\begin{tabular}{lccccc}
\hline$c_{\text {prol }}$ & Q_pr_ii_at random & Q_repl_ii_at random & Q_lf__ii_at random & Q_pr_ii_heuristic_2 & Q_pr_i_heuristic_1 \\
\hline 20,57888656 & 90989,09307692 & 96792,18495670 & 112401,97290374 & 109455,03444509 & 90989,09307692 \\
26,21843164 & 90989,09307692 & 97000,84812439 & 113236,62557449 & 110075,38440308 & 97000,84812439 \\
39,89472481 & 90989,09307692 & 97506,87097200 & 115260,71696492 & 111579,77665272 & 111579,77665272 \\
43,90446372 & 90989,09307692 & 97655,23131138 & 115854,15832244 & 112020,84793196 & 115854,15832244 \\
\hline
\end{tabular}

the real manufacturing time and prolonged manufacturing time reach the value of 90989,09307692 . Table 16 analyses prolonged manufacturing costs and shows intersection points of functions representing implemented manufacturing approaches included in Figure 8 .

Table 16 enables us to notice that Q_pr_i_heuristic_1 matches

Q_pr_ii_at random for $c_{\text {prol }}=20,57888656$

Q_repl_ii_at random for $c_{\text {prol }}=26,21843164$

Q_lfc_ii_at random for $c_{\text {prol }}=43,90446372$

Q_pr_ii_heuristic_2 for $c_{\text {prol }}=39,89472481$

Finally, the comparative cost analysis of the five discussed approaches is carried out. Initial data for this analysis result from calculations taking into account data included in Tables $1-7$. The input data for the analysis include 25 components shown in Tables 17 and 18.
Finally, the results sought for are presented in Table 19 and Figures 9-13 and include the results of the comparative cost simulation for five approaches:

(1) Q_pr_ii_at random;

(2) Q_repl_ii_at_random;

(3) Q_lfc_ii_at_random;

(4) Q_pr_ii_heuristic_2;

(5) Q_pr_i_heuristic_1.

As seen in Table 19 and Figures 9-13 in terms of combined logistics costs and direct production costs the best is the manufacturing approach Q_pr_i_heuristic_1 whereas in terms of parallel production costs the approach Q_pr_ii_at random is the best as these costs are not generated by Q_pr_i_heuristic_1. On the other hand, when replacement costs are taken into account the approach Q_repl_ii_at random wins; however, the lowest lost flow capacity costs are generated in case of Q_lfc_ii_at random. 


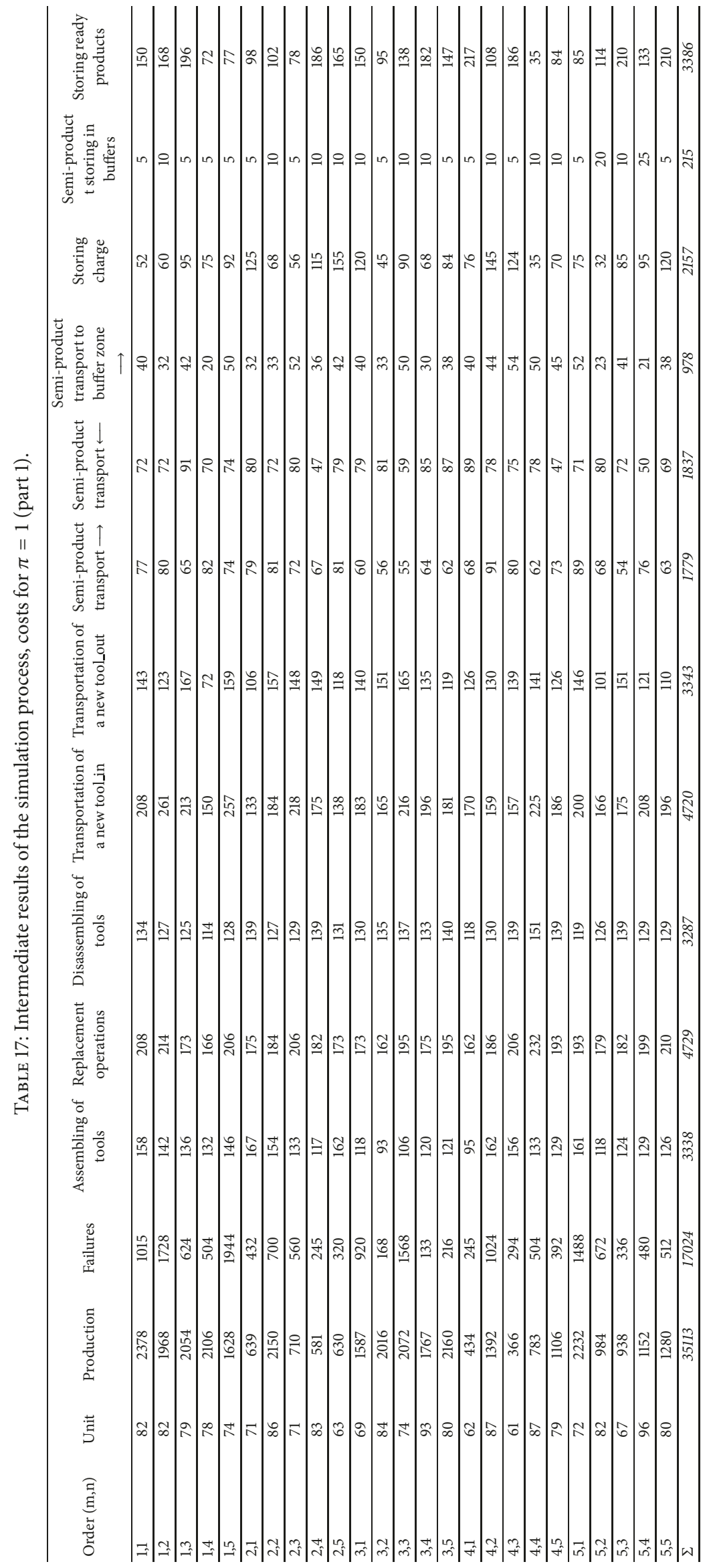




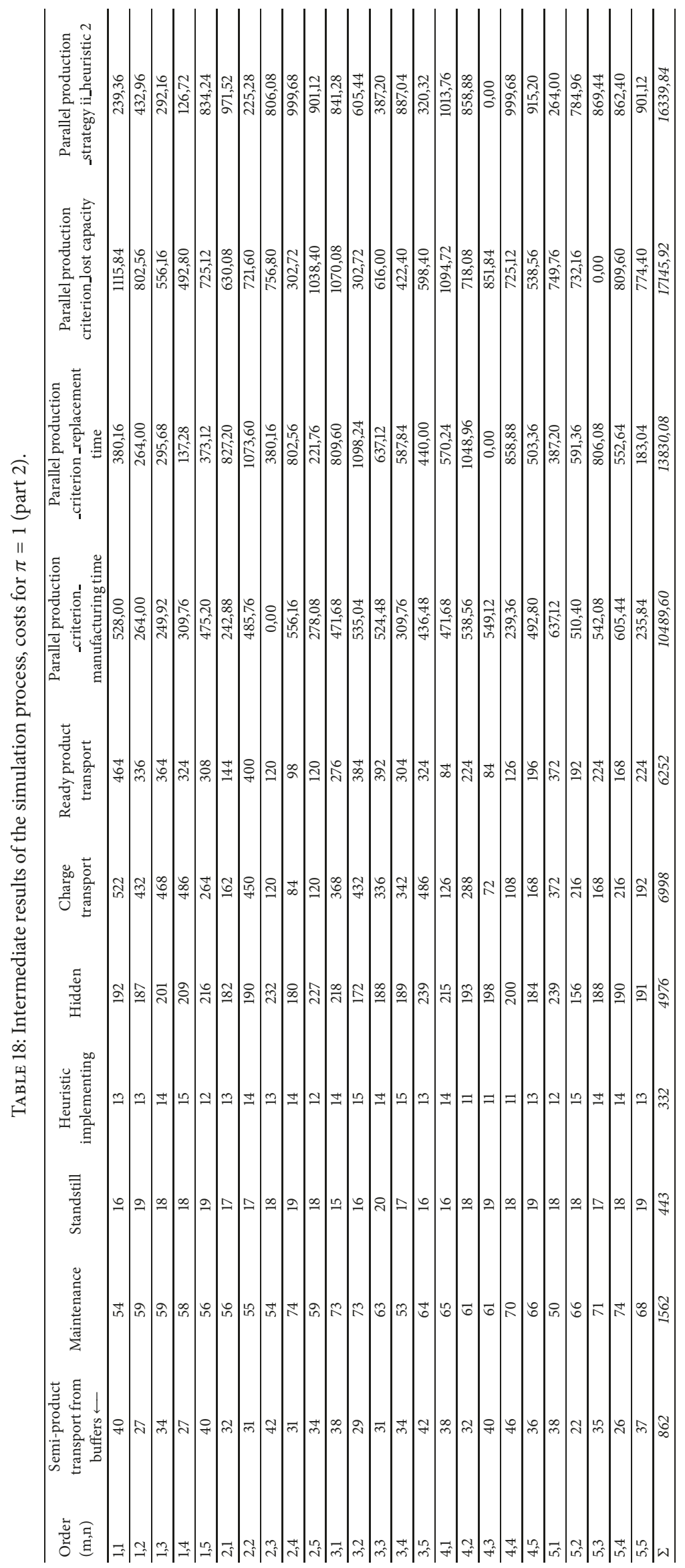




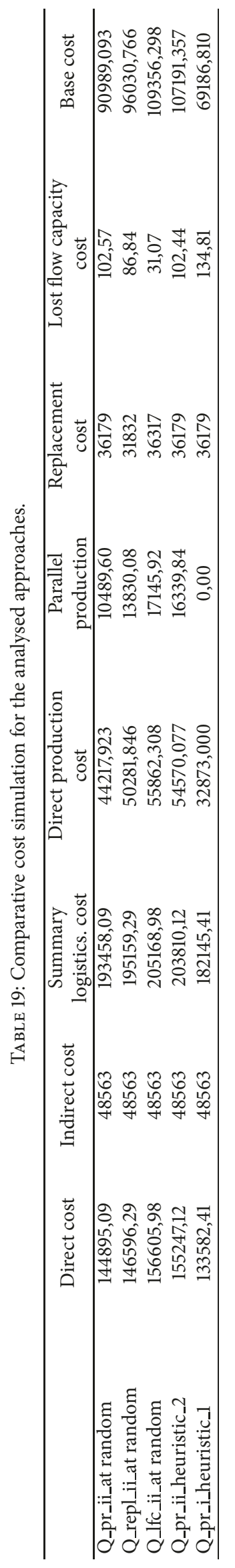




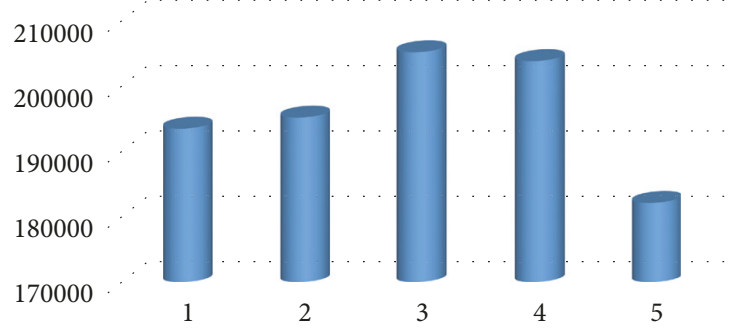

FIgURE 9: Combined logistics costs.

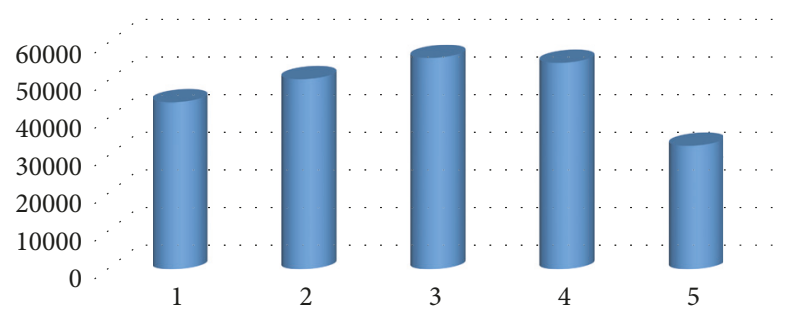

Figure 10: Direct production costs.

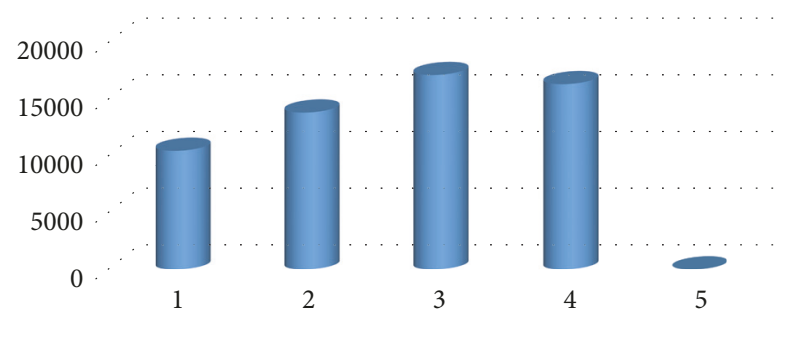

FIGURE 11: Parallel production costs.

\section{Conclusions}

The article emphasises the problem of modelling the complex manufacturing system. The goal is to find such an arrangement of production activities, respectively, production paths for a given set of orders that is effective from the time and cost point of view. First of all, the mathematical model of the system consisting of necessary assumptions creates the basis for building the simulation tool which is implemented to carry out simulations of the manufacturing process. The proposed system with certain data can be evaluated from the point of view of the implemented strategy, heuristic, total production time, effective production time, robotic arm work, total replacement time, lost flow capacity, final flow capacity, total transportation time, system state indicator, system wear indicator, and system flexibility indicator.

The simulation approach may lead to finding the satisfactory solution from the point of view of either the minimal total production time criterion or the minimal replacement time criterion or the minimal lost flow capacity criterion. However, the best achieved result satisfying one analysed criterion does not usually satisfy the others. This leads to the concept of building the multicriteria models which should be devoted to finding the satisfactory solution. Searching for satisfactory solutions is limited by the number of simulations carried out. The bigger the number of simulations, the

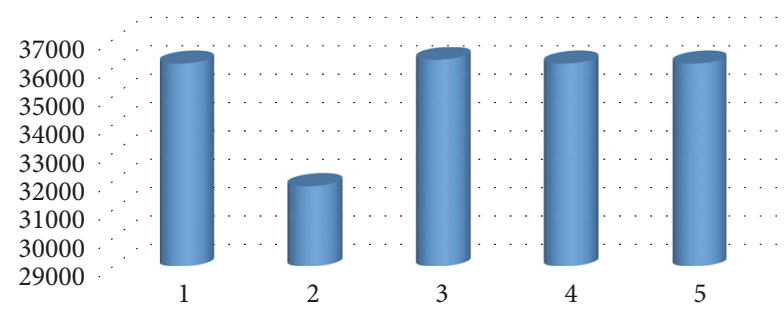

FIGURE 12: Replacement costs.

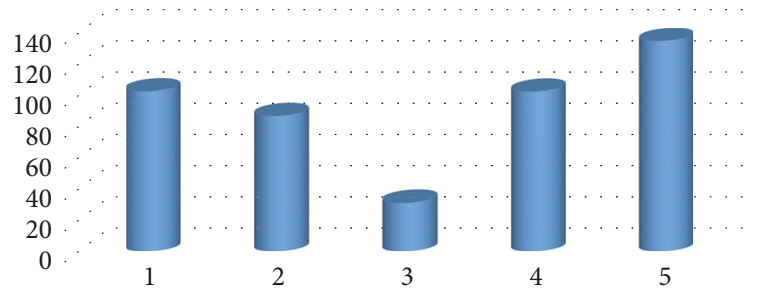

Figure 13: Lost flow capacity costs.

greater the probability of finding a better solution. The only limitation is the time and resources of the software and hardware.

Another important issue is to detect when a manufacturing approach stops being profitable and requires alternating into the one which does not generate excessive costs. This case is illustrated by the analysis of prolonged manufacturing costs. The intersection points inform the system operator that it is the right moment to change the manufacturing approach.

Minimising the order making time remains the main criterion to be discussed; however, verification of order making is to be carried out from the point of view of minimising costs which should result in creating a two-criterion model. On the basis of practical observations of manufacturing processes times of operations must be modified in order to make calculations more precise. Creating a simulation tool on the basis of the above assumptions will enable the authors to carry out simulation experiments analysis of which should result in general conclusions.

Another important factor which needs to be considered is how to control the production process in order to minimise the lost flow capacity of tools; i.e., the best option is to replace fully worn out tools only.

In case of considering prolonged manufacturing up till a certain moment the so far manufacturing approach becomes unprofitable. Therefore, the so-called cost intersection point shows the maximal unit cost of prolonged manufacturing.

Moreover, it is important to bear in mind that the model presented in the paper is only one way of showing how complex it is to prepare the information support for logistics business systems. First of all, it is unavoidable to carry out a thorough analysis of the real existing system which results in specification assumptions as well as the project of the system. These steps are the basis for building the proper simulation tool thoroughly tested and verified before being implemented into the real company. This paper focuses mainly on connecting mathematical modelling of the sample 
hypothetical system which are more than often found in the automotive industry supplying companies as manufacturing tasks are subject to frequent changes; however, costs are to be calculated immediately to enable both the customer and the manufacturer to carry out financial negotiations. There is always a gap to modify the real costs nevertheless; the starting point for subsequent negotiations should be marked.

The research objectives are fully met in the form of the mathematical model of the complex manufacturing system which is the basis for the dedicated simulator. The created simulator is the information tool which enables the simulation of the sample manufacturing process. The results of the simulation process are evaluated in detail in the case study section. Simulation experiments let us draw conclusions concerning functioning of the specific system supplied with sample introductory data. It is necessary to mention that each manufacturing system is autonomous and more than often requires equipping it with the adjusted information tool as the available platform tools lack unavoidable functionalities that need to be implemented. The research in our case focuses on laying foundations for the proper analysis of the modelled problem with the aim of preparing an error-free specification for the dedicated simulator of the manufacturing system. The simulator required thorough testing and validation before it could be employed to solve tasks stated in the paper. However, there are also specification limitations as the modelled system required adequate simplification in order to be able to let us build the simulation tool in its present form. There are numerous possibilities of adding extra functionalities to the specification with the aim of equipping the simulator with them in case of project needs. The approach to solving the problem of modelling a complex manufacturing system with the subsequent simulation experiments seems to be a useful contribution for academics who lecture on modelling and simulation matters. From the practical point of view the article presents a useful method of modelling logistics systems which leads to building a dedicated simulator of the manufacturing system which is an unavoidable information tool for training operators of logistics systems.

Further studies should focus on analysing various logistics systems characterised by their own interdependencies. It is obvious that each of them requires their own specification and project. Nevertheless, it is advisable to focus on the approach which could help develop a simulation tool that is able to carry out simulation experiments in similar types of manufacturing logistics systems.

\section{Symbols}

$C^{\operatorname{assebl}(\pi)}$ :

The matrix of unit assembling costs of tools in the $\pi$-th manufacturing subsystem

$C_{i, j}^{a s s e m b l(\pi)}$ : The unit assembling cost of the tool in the machine placed in the $i$-th row of the $j$-th column in the $\pi$-th subsystem

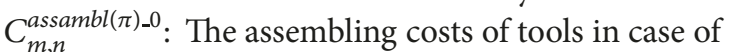
making the order $z_{m, n}^{0}$ in the $\pi$-th manufacturing subsystem
$C^{\text {disa( }(\pi)}$ : The matrix of unit disassembling costs of tools in the $\pi$-th manufacturing subsystem

$C_{i, j}^{\text {disasl }(\pi)}$ : The unit disassembling cost of the tool in the machine placed in the $i$-th row of the $j$-th column in the $\pi$-th subsystem

$C_{m, n}^{\text {disas }(\pi)_{-} 0}$ : The disassembling costs of tools in case of making the order $z_{m, n}^{0}$ in the $\pi$-th manufacturing subsystem

$C_{m, n}^{p r(\pi)}: \quad$ The matrix of unit manufacturing costs in the $\pi$-th manufacturing subsystem

$C_{(m, n) /(i, j)}^{p r(\pi)}:$ The unit cost of manufacturing the $n$-th order for the $m$-th customer in the $j$-th machine in the $i$-th row in the $\pi$-th manufacturing subsystem

$C_{m, n}^{\sum p r(\pi)_{-0} 0}$ : The cost of manufacturing the order $z_{m, n}^{0}$ in the $\pi$-th manufacturing subsystem

$C^{\text {fail }(\pi)}: \quad$ The matrix of possible maintenance costs due to failure of the $\pi$-th manufacturing subsystem

$C_{m, n}^{f a i l(\pi)}: \quad$ The unit cost of possible maintenance procedures due to failure of tools while making the $n$-th order for the $m$-th customer in the $\pi$-th manufacturing subsystem

$C_{m, n}^{f a i l(\pi)_{-} 0}:$ The costs of possible failure maintenance in case of making the order $z_{m, n}^{0}$ in the $\pi$-th manufacturing subsystem

$C_{\longrightarrow}^{\operatorname{tr}(\pi)}: \quad$ The matrix of unit transportation costs for semiproducts from machines to the key point in the $\pi$-th manufacturing subsystem

$C_{(i, j) / \rightarrow \bullet}^{t r(\pi)}:$ The unit transportation time of products from the machine placed in the $i$-th row of the $j$-th column to the key point in the $\pi$-th subsystem

$C_{(m, n) / \rightarrow}^{\operatorname{tr}(\pi) \_0}:$ The transportation costs for semiproducts from machines to the key point in case of making the order $z_{m, n}^{0}$ in the $\pi$-th manufacturing subsystem

$C_{. \rightarrow}^{\operatorname{tr}(\pi)}: \quad$ The matrix of unit transportation costs for semiproducts from the key point in the $\pi$-th manufacturing subsystem

$C_{\bullet \rightarrow\left(i, j^{\prime}\right)}^{t r(\pi)}:$ The unit transportation cost of products from the key point to the machine placed in placed in the row $i$ of the column $j^{\prime}$ in the $\pi$-th subsystem

$C_{(m, n) / \bullet \longrightarrow}^{t r(\pi) \_0}:$ The transportation costs for semiproducts from the key point to machines in case of making the order $z_{m, n}^{0}$ in the $\pi$-th manufacturing subsystem

$C_{\rightarrow \text { buffer }}^{\operatorname{tr}(\pi)}: \quad$ The matrix of unit transportation costs for semiproducts transported from machines to buffer stores in the $\pi$-th manufacturing subsystem

$C_{(i, j) \rightarrow b_{j}}^{t r(\pi)}$ : The unit transportation cost of a product transported from the machine placed in the $i$-th row of the $j$-th column to the $j$-th buffer store in the $\pi$-th subsystem 
$C_{\longrightarrow \text { buffer }}^{\operatorname{tr}(\pi) \_0}: \quad$ The transportation costs for semiproducts transported to buffer stores from machines in case of making the order $z_{m, n}^{0}$ in the $\pi$-th manufacturing subsystem

$C_{s t}^{(\pi) w}: \quad$ The matrix of unit costs of storing charge materials in the $\pi$-th manufacturing subsystem

$C_{w /(m, n)}^{(\pi) s t}: \quad$ The storing cost of the $w$-th charge material for making the $n$-th product for the $m$-th customer in the machine placed in the $\pi$-th plant

$C^{(\pi) b u f \_s t}$ : The matrix of unit costs of storing semiproducts in the buffer stores in the $\pi$-th manufacturing subsystem

$C_{m, n}^{(\pi) b u f_{-} s t}$ : The storing cost of the $n$-th semiproduct made for the $m$-th customer in the available buffer stores in the $\pi$-th manufacturing subsystem

$C_{\max }^{(\pi) b u f_{-} s t}$ : Costs of storing semiproducts in the buffer stores in the $\pi$-th manufacturing subsystem

$C^{(\pi) s t_{-} z}$ : The matrix of unit costs of storing ready products in the $\pi$-th manufacturing subsystem

$C_{m, n}^{(\pi) s t_{-} z}: \quad$ The unit storing cost of the $n$-th ready product made for the $m$-th customer in the ready product store in the $\pi$-th manufacturing subsystem

$C^{(\pi) \text { storage }}$ The total storing costs in the ready product store in the $\pi$-th manufacturing subsystem

$C^{(\pi) \text { main }}$ : The matrix of maintenance costs in the $\pi$-th manufacturing subsystem

$C_{i, j}^{(\pi) \text { main }}:$ The maintenance cost of the machine placed in the $i$-th row of the $j$-th column in the $\pi$-th manufacturing plant

$C^{(\pi) \text { still }}$ : The matrix of standstill costs in the $\pi$-th manufacturing subsystem

$C_{(\mathrm{i}, \mathrm{j}) /(\mathrm{m}, \mathrm{n})}^{(\pi) \text { still }}:$ The unit standstill costs in case of making the $n$-th product for the $m$-th customer in the machine placed in the $i$-th row of the $j$-th column in the $\pi$-th manufacturing subsystem

$C^{(\pi) \text { heur }}$ : The matrix of costs of implementing heuristic algorithms in the $\pi$-th manufacturing subsystem

$C_{(i, j) /(m, n)}^{(\pi)\lrcorner \text { heur }}$ : The cost of implementing heuristic algorithms in case of making the $n$-th product made for the $m$-th customer in the machine placed in the $i$-th row of the $j$-th column in the $\pi$-th manufacturing subsystem

$C^{(\pi) \_h c}: \quad$ The matrix of hidden operating costs in the $\pi$-th manufacturing subsystem

$C_{(\mathrm{i}, \mathrm{j}) /(\mathrm{m}, \mathrm{n})}^{(\pi) / \mathrm{h}}$ : The hidden unit cost in case of making the $n$-th product for the $m$-th customer in the machine placed in the $i$-th row of the $j$-th column of the $\pi$-th manufacturing subsystem
$C_{\text {base_ } \delta}: \quad$ The base manufacturing costs for the given $\delta$-th approach

$C_{b u f f e r \longrightarrow}^{t r(\pi)}: \quad$ The matrix of unit transportation costs for semiproducts transported from buffer stores to machines in the $\pi$-th manufacturing subsystem

$C_{b_{j} \longrightarrow\left(i, j^{\prime}\right)}^{\operatorname{tr}(\pi)}:$ The transportation cost of a product from the $j$-th buffer store to machine placed in the $i$-th row of the column $j^{\prime}$ in the $\pi$-th subsystem

$C_{\text {buffer } \longrightarrow}^{\operatorname{tr}(\pi)_{0} 0}: \quad$ The unit transportation costs for semiproducts transported from buffer stores to machines in case of making the order in the $\pi$-th manufacturing subsystem

$C_{c h \longrightarrow}^{t r(\pi)}: \quad \quad$ The matrix of unit transportation costs for the charge material from the store of charges to machines in the $\pi$-th manufacturing subsystem

$C_{c h \longrightarrow(i, j)}^{t r(\pi)_{-}(m, n)}: \quad$ The unit transportation cost of the charge material to the machine placed in the $i$-th row of the $j$-th column in case of making the $n$-th order for the $m$-th customer in the $\pi$-th subsystem

$C_{c h \longrightarrow}^{t r(\pi)_{-}(m, n)_{-} 0}: \quad$ The total transportation costs of charge material transported from the charge store to machines in case of making the order $z_{m, n}^{0}$ in the $\pi$-th manufacturing subsystem $C_{(i, j) \longrightarrow z_{s t}}^{\operatorname{tr}(\pi)}: \quad$ The matrix of unit transportation costs of ready products to the product store in the $\pi$-th manufacturing subsystem

$C_{(i, j) \longrightarrow z_{s t}}^{t r(\pi)_{(}(m, n)}: \quad$ The unit transportation cost of the ready product transported the product store in case of making the $n$-th order for the $m$-th customer in the $\pi$-th subsystem

$C_{(i, j) \longrightarrow z_{s t}}^{\operatorname{tr}(\pi)_{-}(m, n){ }_{-} 0}: \quad$ The transportation cost of a ready product transported to the product store in case of making the order $z_{m, n}^{0}$ in the $\pi$-th manufacturing subsystem

$C_{\mathrm{Q} \_}^{\operatorname{par} \delta}: \quad \quad \quad$ The matrix of parallel manufacturing costs in the $\pi$-th manufacturing subsystem

$C_{\mathrm{Q}_{-} \delta_{-}(m, n) /\left(m^{\prime}, n^{\prime}\right)}^{\operatorname{par}(\pi)}:$ The manufacturing cost of making the $n$-th order for the $m$-th customer and the $n^{\prime}$-th order for the $m^{\prime}$-th customer in a parallel way in the $\pi$-th subsystem with the use of the $\delta$-th criterion

$C^{\alpha / \pi}$ : $\quad$ The matrix of costs of replacement operations in the $\pi$-th manufacturing subsystem

$C_{i, j}^{\alpha / \pi}: \quad \quad$ The replacement operation cost of the $\alpha$-th tool in the machine placed in the $i$-th row of the $j$-th column in the $\pi$-th subsystem

$C^{\alpha_{i n}}$ : The matrix of transportation costs for new tools which are stored in the storing place for tools in the $\pi$-th manufacturing subsystem 
$C_{i, j}^{\alpha_{i n} / \pi}: \quad$ The transportation cost of the new $\alpha$-th type tool from the storing place to the machine placed in the $i$-th row of the $j$-th column in the $\pi$-th subsystem

$C^{\alpha_{\text {out }} / \pi}: \quad$ The matrix of transportation costs for worn out tools to the storing place for used tools in the $\pi$-th manufacturing subsystem

$C_{i, j}^{\alpha_{\text {out }} / \pi}: \quad$ The transportation cost of the worn out $\alpha$-th type tool from the machine placed in the $i$-th row of the $j$-th column to the storing place for worn out tools in the $\pi$-th subsystem

$C_{\text {prol_o } \delta}^{\pi}: \quad$ The costs of prolonged manufacturing in the $\pi$-th subsystem

$C_{\text {prol }}^{\pi}: \quad$ The unit cost of prolonged manufacturing in the $\pi$-th subsystem

$C_{\text {repl }}^{\pi}: \quad$ The cost of the replacement process in the $\pi$-th manufacturing subsystem

$C_{\text {repl }}^{\pi}: \quad$ The unit cost of the replacement process in the $\pi$-th manufacturing subsystem

$C_{l f c}^{\pi}: \quad$ The cost of the lost flow capacity in the $\pi$-th manufacturing subsystem

$C_{l f c}^{\pi}: \quad$ The unit cost of the lost flow capacity after completing the manufacturing process in the $\pi$-th manufacturing subsystem

$C_{f c}^{\pi}: \quad$ The company cost of the $\pi$-th manufacturing subsystem

$C_{f i x}^{\pi}: \quad$ The fixed manufacturing cost of the $\pi$-th manufacturing subsystem

$C_{o p-(Q-\delta)}^{\pi}:$ The logistics costs of the $\pi$-th manufacturing subsystem

$C_{\mathrm{Q} \delta}^{\pi}: \quad$ The approach cost of the $\pi$-th manufacturing subsystem

$D: \quad$ The matrix of routes

$d_{(m, n), j}: \quad$ The number of the $i$-th row through which the $n$-th semiproduct for the $m$-th customer is passed throughout the manufacturing process

E: The general matrix of structure of the manufacturing system

$e_{(m, n) /(i, j)}^{\pi}:$ A machine located in the $i$-th row of the $j$-th column in the $\pi$-th subsystem dedicated to performing a manufacturing operation on the $n$-th order for the $m$-th customer with the use of a certain type of tool

G: $\quad$ The base life matrix of tools used in the manufacturing system of a new brand set of tools used to manufacture elements of the order matrix

$g_{n /(i, j)}^{\alpha}: \quad$ The base number of units of the $n$-th product which can be manufactured in the machine in the $i$-th row of the $j$-th column before the $\alpha$-th dedicated tool is completely worn out and requires an immediate replacement

$H: \quad$ The adjustment matrix of tools to machines $h_{i, j}^{\alpha}: \quad$ The adjustment of the $\alpha$-th tool to the machine placed in the $i$-th row of the $j$-th column

$i$ The number of the row in the system, $i=1, \ldots, I$

$j: \quad$ The number of the column in the system, $j=1, \ldots, J$

$k: \quad$ The stage number, $k=1, \ldots, K$

$K: \quad$ The total number of system decisions necessary to produce all order matrix elements approved for manufacturing at the stage $k=0$

$m: \quad$ The number of the customer, $m=1, \ldots, M$

$n: \quad$ The type of order, $n=1, \ldots, N$

$\min \tau_{\delta}^{\pi \text { end }}$ : The time of completing making the order by the manufacturing approach minimising the manufacturing time in the $\pi$-th subsystem

$P_{n}^{(\alpha / \pi)-k}: \quad$ The capacity matrix of the $\pi$-th manufacturing subsystem

$p_{n /(i, j)}^{(\alpha / 2)-k}: \quad$ The number of the $n$-th product units which still can be made in the machine in the $i$-th row of the $j$-th column with the use of the $\alpha$-th tool in the $\pi$-th subsystem at the $k$-th state

$Q_{l f c}: \quad$ The criterion of lost flow capacity of tools

$Q_{p r}: \quad$ The time criterion of the production process

$Q_{\text {repl }}: \quad$ The criterion of the replacement process

$S_{n}^{(\alpha / \pi)-k}$ : The matrix of state of the the $\pi$-th manufacturing subsystem

$S_{n /(i, j)}^{(\alpha /)_{-}-k}: \quad$ The number of units of the $n$-th product already made in the machine in the $i$-th row of the $j$-th column with the use of the $\alpha$-th tool in the $\pi$-th subsystem at the $k$-th state

$T^{\text {assembl( } \pi)}$ : The matrix of assembling times of tools in the $\pi$-th subsystem

$\tau_{(i, j)}^{a s s e m b l(\pi)}$ : The assembling time of the tool in the machine placed in the $i$-th row of the $j$-th column in the $\pi$-th subsystem

$T^{\text {disas }(\pi)}$ : The matrix of disassembling times of tools in the $\pi$-th subsystem

$\tau_{(i, j)}^{\text {disas }(\pi)}: \quad$ The disassembling time of the tool in the machine placed in the $i$-th row of the $j$-th column in the $\pi$-th subsystem

$T^{p r(\pi)}: \quad$ The matrix of production times in the $\pi$-th manufacturing system

$\tau_{(m, n) /(i, j)}^{p r(\pi)}: \quad$ The production time of the $n$-th type of product for the $m$-th customer in the machine placed in the $i$-th row of the $j$-th column in the $\pi$-th subsystem

$\tau_{\delta}^{\pi-e n d}: \quad$ The time of completing making the order by the $\delta$-th approach in the $\pi$-th subsystem

$\tau_{\text {repl }}^{\pi}: \quad$ The total replacement time in the $\pi$-th manufacturing subsystem

$T_{\rightarrow \rightarrow \bullet}^{t r(\pi)}: \quad$ The matrix of transportation times for semiproducts from machines to the key manipulation point in the $\pi$-th subsystem 
$\tau_{(i, j) / \rightarrow \bullet}^{\operatorname{trl}(\pi)}:$ The transportation time of products from the machine placed in the $i$-th row of the $j$-th column to the key point in the $\pi$-th manufacturing system

$T_{. \longrightarrow}^{t r(\pi)}: \quad$ The matrix of transportation times for semiproducts from the key point to machines in the $\pi$-th subsystem

$\tau_{. \longrightarrow /\left(i, j^{\prime}\right)}^{\operatorname{tr}(\pi)}:$ The transportation time of products from the key point to the machine placed in to the machine placed in the row $i$ of the column $j^{\prime}$ in the $\pi$-th manufacturing system

$T_{\longrightarrow \text { buffer }}^{\operatorname{tr}(\pi)}: \quad$ The matrix of transportation times for semiproducts from machines to their buffer stores in the $\pi$-th subsystem

$\tau_{(i, j) \rightarrow b_{j}}^{\operatorname{tr}(\pi)}: \quad$ The transportation time of a product from the machine placed in the $i$-th row of the $j$-th column to the $j$-th buffer store in the $\pi$-th manufacturing system

$T_{\text {buffer } \rightarrow}^{t r(\pi)}: \quad$ The matrix of transportation times for semiproducts from buffer stores to machines in the $\pi$-th subsystem

$\tau_{b_{j} \longrightarrow\left(i, j^{\prime}\right)}^{\operatorname{tr}(\pi)}:$ The transportation time of a product from the $j$-th buffer store to machine placed in the $i$-th row of the column $j^{\prime}$ in the $\pi$-th manufacturing system

$T_{c h \longrightarrow}^{\operatorname{tr}(\pi)}: \quad$ The matrix of unit transportation times for charge material from the store of charges to machines in the $\pi$-th subsystem

$\tau_{c h \longrightarrow(i, j)}^{\operatorname{tr}(\pi)_{-}(m, n)}:$ The unit transportation time of the charge material to the machine placed in the $i$-th row of the $j$-th column in case of making the $n$-th order for the $m$-th customer in the $\pi$-th manufacturing system

$T_{\stackrel{\operatorname{tr}(\pi)}{\longrightarrow} z_{s t}}: \quad$ The matrix of unit transportation times for ready products to the product store in the $\pi$-th subsystem

$\tau_{(i, j) \longrightarrow z_{s t}}^{\operatorname{tr}(\pi)_{-}(m, n)}:$ The unit transportation time of the ready product from the machine placed in the $i$-th row of the $j$-th column to the product store in case of making the $n$-th order for the $m$-th customer in the $\pi$-th manufacturing system

$T_{w}^{(\pi) s t}$ : The matrix of times of storing charge materials in the $\pi$-th subsystem

$\tau_{w /(m, n)}^{(\pi) s t}: \quad$ The time of storing the $w$-th charge material for making the $n$-th product for the $m$-th customer in the charge store for the $\pi$-th plant

$T^{(\pi) b u f \_s t}$ : The matrix of times of storing semiproducts in buffer stores in the $\pi$-th subsystem

$\tau_{m, n}^{(\pi) b u f_{s} s t}$ : The average storing time of the $n$-th semiproduct made for the $m$-th customer in the available buffer store in the $\pi$-th plant

$T^{(\pi) s t_{-} z}$ : The matrix of times of storing ready products in the $\pi$-th subsystem $\tau_{m, n}^{(\pi) s t z}: \quad$ The storing time of the $n$-th ready product made for the $m$-th customer in the ready product store of the $\pi$-th manufacturing system

$T_{\mathrm{Q} \_\delta}^{\text {par }(\pi)}: \quad \quad$ Matrix of parallel manufacturing times in the $\pi$-th subsystem with the use of the $\delta$-th criterion

$\tau_{\mathrm{Q}_{-} \delta_{-}(m, n) /\left(m^{\prime}, n^{\prime}\right)}^{\operatorname{par}(\pi)}:$ The parallel manufacturing time of making the $n$-th order for the $m$-th customer and the $n^{\prime}$-th order for the $m^{\prime}$-th customer in the $\pi$-th subsystem with the use of the $\delta$-th criterion

$\tau_{Q_{-} \delta_{-}(m, n)}^{\operatorname{par}(\pi)_{-} \text {start }}: \quad$ The moment of beginning making the $n$-th order for the $m$-th customer and the $n^{\prime}$-th order for the $m^{\prime}$-th customer in the $\pi$-th subsystem with the use of the $\delta$-th criterion

$\tau_{Q_{-} \delta_{-}(m, n)}^{\operatorname{par}(\pi)_{\text {end }}}: \quad$ The moment of finishing making the $n$-th order for the $m$-th customer and the $n^{\prime}$-th order for the $m^{\prime}$-th customer in the $\pi$-th subsystem with the use of the $\delta$-th criterion

$T^{\alpha / \pi}: \quad \quad$ The matrix of times of replacement operations

$\tau_{i, j}^{\alpha / \pi}: \quad$ The replacement operation time of the $\alpha$-th tool in the machine placed in the $i$-th row of the $j$-th column in the $\pi$-th subsystem

$T^{\alpha_{i n}}$ : The matrix of transportation times of new tools which are stored in the storing place for tools in the $\pi$-th subsystem

$$
\tau_{i, j}^{\alpha_{i n} / \pi}:
$$

The transportation time of the new $\alpha$-th type tool from the storing place to the machine placed in the $i$-th row of the $j$-th column in the $\pi$-th subsystem

$T^{\alpha_{\text {out }} / \pi}$ : $\quad$ The matrix of transportation times for worn out tools to the storing place for used tools in the $\pi$-th subsystem

$\tau_{i, j}^{\alpha_{\text {out }} / \pi}:$

$x_{m, n}^{k}$

$Z^{k}$ :

$z_{m, n}^{k}:$

$z_{\mu, \eta}^{k}$

$\alpha:$

$\beta$ : $\Gamma^{\text {fail }(\pi)}$
The transportation time of the worn out $\alpha$-th type tool from the machine placed in the $i$-th row of the $j$-th column to the storing place for worn out tools in the $\pi$-th subsystem

The number of units of the $n$-th order for the $m$-th customer is made at the $k$-th stage

The matrix of orders at the $k$-th stage

The state of the $n$-th order set by the $m$-th customer at the $k$-th stage

The element to be made in the manufacturing zone at the $k$-th stage The $\alpha$-th tool, $\alpha=1, \ldots, A$ The $\beta$-th operation, $\beta=1, \ldots, B$ The matrix of probability coefficients of failure of tools in the $\pi$-th manufacturing subsystem 
$\gamma_{m, n}^{f a i l(\pi)}$ : The probability coefficient of failure of tools in case of making the $n$-th order for the $m$-th customer in the $\pi$-th manufacturing subsystem

$\Delta \tau_{\text {prol_s}}^{\pi}:$ The time of prolonged manufacturing according for the $\delta$-th approach in the $\pi$-th subsystem

Y: $\quad$ The matrix of adjustment of tools to operations

$v_{\alpha, \beta}: \quad$ Adjustment of the $\alpha$-th tool to the $\beta$-th operation

$\Theta^{0}$ : $\quad$ The matrix of order priority

$\theta_{m, n}^{k}: \quad$ The priority of the manufacturing task for the $m$-th customer who ordered the $n$-th product at the $k$-th stage

$\Xi^{\pi}: \quad$ The vector of buffer zones in the $\pi$-th manufacturing subsystem

$\xi_{j}^{\pi}: \quad$ The buffer zone behind the machine placed in the $j$-th column in the $\pi$-th manufacturing plant

$\varsigma_{l f c}^{\pi}: \quad$ The number of lost flow capacity units after completing the manufacturing process in the $\pi$-th manufacturing subsystem

$\varsigma_{f i x}^{\pi}: \quad$ The coefficient of stable costs in the $\pi$-th manufacturing subsystem

П: $\quad$ The total number of logistics manufacturing centres

$\pi: \quad$ The $\pi$-th logistics manufacturing centre.

\section{Data Availability}

The data used to support the findings of this study are included within the article.

\section{Conflicts of Interest}

The authors declare that they have no conflicts of interest.

\section{Acknowledgments}

This paper was supported by the Project SGS/8/2018, "Advanced Methods and Procedures of Business Processes Improvement" at the Silesian University in Opava, School of Business Administration in Karvina.

\section{References}

[1] M. Dumas, M. La Rosa, J. Mendling, and H. A. Reijers, Fundamentals of Business Process Management, Springer, 2018.

[2] M. K. Albzeirat, M. I. Hussain, R. Ahmad, F. M. Al-Saraireh, and I. Ahmad, "A novel mathematical logic for improvement using lean manufacturing practices," Journal of Advanced Manufacturing Systems, vol. 17, no. 3, pp. 391-413, 2018.

[3] F. Aqlan and L. Al-Fandi, "Prioritizing process improvement initiatives in manufacturing environments," International Journal of Production Economics, vol. 196, pp. 261-268, 2018.

[4] S. Parthasarathy and S. Sharma, "Efficiency analysis of ERP packages - A customization perspective," Computers in Industry, vol. 82, pp. 19-27, 2016.
[5] P. Jaferian, K. Hawkey, A. Sotirakopoulos, M. Velez-Rojas, and K. Beznosov, "Heuristics for evaluating IT security management tools," Human-Computer Interaction, vol. 29, no. 4, pp. 311-350, 2014.

[6] I. Madanhire and C. Mbohwa, "Enterprise resource planning (ERP) in improving operational efficiency: case study," in Proceedings of the 13th Global Conference on Sustainable Manufacturing, GCSM '15, vol. 40, pp. 225-229, 2015.

[7] G. Marodin, A. G. Frank, G. L. Tortorella, and T. Netland, "Lean product development and lean manufacturing: testing moderation effects," International Journal of Production Economics, vol. 203, pp. 301-310, 2018.

[8] T. NG and M. Ghobakhloo, "What derives lean manufacturing effectiveness: An interpretive structural mode," International Journal of Advanced and Applied Sciences, vol. 4, no. 8, pp. 104111, 2017.

[9] A. G. Chofreh, F. A. Goni, and J. J. Klemeš, "Sustainable enterprise resource planning systems implementation: a framework development," Journal of Cleaner Production, vol. 198, pp. 1345$1354,2018$.

[10] A. Ustundag and E. Cevikcan, "Industry 4.0: Managing The Digital Transformation," Springer Series in Advanced Manufacturing, 2017.

[11] A. Theorin, K. Bengtsson, J. Provost et al., "An event-driven manufacturing information system architecture for Industry 4.0," International Journal of Production Research, vol. 55, no. 5, pp. 1297-1311, 2017.

[12] C. Thuemmler and C. Bai, Health 4.0: How Virtualization and Big Data are Revolutionizing Healthcare, Springer, 1st edition, 2017.

[13] K. Shamn, S. Cang, N. H. Yu, and Y. Li, "Management approaches for industry 4.0 a human resource management perspective," in Proceedings of the IEEE Congress on Evolutionary Computation (CEC '16), pp. 5309-5316, 2016.

[14] F. Hecklau, M. Galeitzke, S. Flachs, and H. Kohl, "Holistic approach for human resource management in Industry 4.0," in Proceedings of the 6th CIRP Conference on Learning Factories, pp. 1-6, 2016

[15] G. Joglekar, "Incorporating enhanced decision-making capabilities into a hybrid simulator for scheduling of batch processes," Processes, vol. 4, no. 3, 2016.

[16] J. G. Moser, "Integration of artificial-intelligence and simulation in a comprehensive decision-support system," Simulation, vol. 47, no. 6, pp. 223-229, 1986.

[17] J. E. Abu Qudeiri, "Production simulator system for flexible routing optimization in flexible manufacturing systems," Proceedings of the Institution of Mechanical Engineers, Part B: Journal of Engineering Manufacture, vol. 231, no. 7, pp. 1237-1247, 2017.

[18] M. M. Tavakoli, H. Haleh, and M. Mohammadi, "A mathematical model for scheduling of production process and allocation of an automatic guided vehicle in a flexible manufacturing system," International Journal of Engineering Systems Modelling and Simulation, vol. 10, no. 2, pp. 125-131, 2018.

[19] M. Aghajani, A. Keramati, R. T. Moghadam, and S. S. Mirjavadi, "A mathematical programming model for cellular manufacturing system controlled by kanban with rework consideration," The International Journal of Advanced Manufacturing Technology, vol. 83, no. 5-8, pp. 1377-1394, 2016.

[20] H. Zhang, B. Zhu, Y. Li, O. Yaman, and U. Roy, "Development and utilization of a process-oriented information model for 
sustainable manufacturing," Journal of Manufacturing Systems, vol. 37, pp. 459-466, 2015.

[21] J. Koch, N. Michels, and G. Reinhart, "Context model design for a process-oriented Manufacturing Change Management," in Proceedings of the 48th CIRP Conference on Manufacturing Systems, vol. 21, pp. 33-38, 2016.

[22] J. F. Shapiro, "Modeling the Supply Chain," South-Western CollegePub, 2006.

[23] T. Y. Li, T. He, and H. C. Xu, "Value-oriented operation process model for service supply chain," Computer Integrated Manufacturing Systems, vol. 21, no. 1, pp. 235-245, 2015.

[24] M. Wooldridge, An Introduction to Multi Agent Systems, Wiley, 2009.

[25] C. H. Yang, R. X. Yang, T. T. Xu, and Y. X. Li, "Negotiation model and tactics of manufacturing enterprise supply chain based on multi-agent," Advances in Mechanical Engineering, vol. 10, no. 7, 2018.

[26] G. Jiang, S. Liu, W. Liu, and Y. Xu, "Agent-based modeling and simulation of the decision behaviors of e-retailers," Industrial Management \& Data Systems, vol. 118, no. 5, pp. 1094-1113, 2018.

[27] E. J. Macías and M. P. de la Parte, "Simulation and optimisation of logistic and production systems using discrete and continuous petri nets," Simulation-transactions of the Society for Modeling and Simulation International, vol. 80, no. 3, pp. 143$152,2004$.

[28] R. Davidrajuh, "A new petri nets based approach for modeling of discrete manufacturing system," in Proceedings of the Intelligent Systems in Production Engineering and Maintenance (ISPEM '17), pp. 109-120, 2018.

[29] S. A. Shah, E. L. J. Bohez, K. Shah, I. ul Haq, K. Azam, and S. Anwar, "Colored Petri net model for significant reduction of invariants in flexible manufacturing systems," The International Journal of Advanced Manufacturing Technology, vol. 88, no. 5-8, pp. 1775-1787, 2017.

[30] M. Dotoli, M. P. Fanti, A. M. Mangini, and G. Tempone, "Fuzzy multi-objective optimisation for network design of logistic and production systems," in Proceedings of the 10th IEEE International Conference on Emerging Technologies and Factory Automation, pp. 475-482, 2005.

[31] I. Djelloul, Z. Sari, and K. Latreche, "Uncertain fault diagnosis problem using neuro-fuzzy approach and probabilistic model for manufacturing systems," Applied Intelligence, vol. 48, no. 9, pp. 3143-3160, 2018.

[32] B. Chramcov and J. Balate, "Model-building for time series of heat demand," in Proceedings of the Annals of DAAAM for 2009 and 20th International DAAAM Symposium "Intelligent Manufacturing and Automation: Focus on Theory, Practice and Education", pp. 195-196, Austria, November 2009.

[33] R. Bucki and P. Suchánek, "The method of logistic optimization in E-commerce," Journal of Universal Computer Science, vol. 18, no. 10, pp. 1238-1258, 2012.

[34] S. Özpeynirci, "A heuristic approach based on time-indexed modelling for scheduling and tool loading in flexible manufacturing systems," The International Journal of Advanced Manufacturing Technology, vol. 77, no. 5-8, pp. 1269-1274, 2015.

[35] J. Ehm, M. Freitag, and E. M. Frazzon, "A heuristic optimisation approach for the scheduling of integrated manufacturing and distribution systems," Procedia CIRP, vol. 57, pp. 357-361, 2016.

[36] H. Lei, K. Xing, L. Han, and Z. Gao, "Hybrid heuristic search approach for deadlock-free scheduling of flexible manufacturing systems using Petri nets," Applied Soft Computing, vol. 55, pp. 413-423, 2017.
[37] A. Biele and L. Mönch, "Hybrid approaches to optimize mixedmodel assembly lines in low-volume manufacturing," Journal of Heuristics, vol. 24, no. 1, pp. 49-81, 2018.

[38] A. Yebi and B. Ayalew, "Hybrid modeling and robust control for layer-by-layer manufacturing processes," IEEE Transactions on Control Systems Technology, vol. 25, no. 2, pp. 550-562, 2017.

[39] D. Lechevalier, S. Hudak, A. K. Ronay, Y. T. Lee, and S. Foufou, "A neural network meta-model and its application for manufacturing," in Proceedings of the 3rd IEEE International Conference on Big Data, IEEE Big Data 2015, pp. 1428-1435, USA, November 2015.

[40] G. D. Putnik, L. Varela, and V. Modrák, "Intelligent collaborative decision-making models, methods, and tools," Mathematical Problems in Engineering, vol. 2018, Article ID 9627917, 2 pages, 2018.

[41] R. Farahani, S. Rezapour, and L. Kardar, Logistics Operations and Management: Concepts and Models, Elsevier, 1st edition, 2011.

[42] B. Chramcov, R. Bucki, and M. Trzopek, "Model based control of production flows in the serial logistic process," in Proceedings of the 34th International Conference on Mathematical Methods in Economics, pp. 313-318, 2016.

[43] H. Kaur and S. P. Singh, "Heuristic modeling for sustainable procurement and logistics in a supply chain using big data," Computers and Operations Research, vol. 98, pp. 301-321, 2018.

[44] C.-C. Chen and P. Schonfeld, "A hybrid heuristic technique for optimal coordination in intermodal logistics scheduling," International Journal of Shipping and Transport Logistics, vol. 9, no. 4, pp. 475-499, 2017.

[45] A. Moreno, D. Alem, and D. Ferreira, "Heuristic approaches for the multiperiod location-transportation problem with reuse of vehicles in emergency logistics," Computers \& Operations Research, vol. 69, pp. 79-96, 2016.

[46] K. M. S. Alzaidi, O. Bayat, and O. N. Ucan, "A heuristic approach for optimal planning and operation of distribution systems," Journal of Optimization, vol. 2018, Article ID 6258350, 19 pages, 2018. 


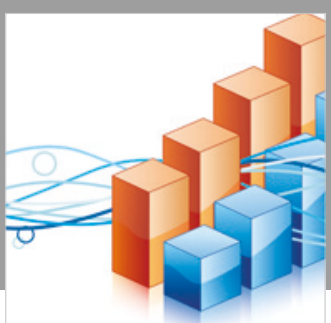

Advances in

Operations Research

\section{-n-m}
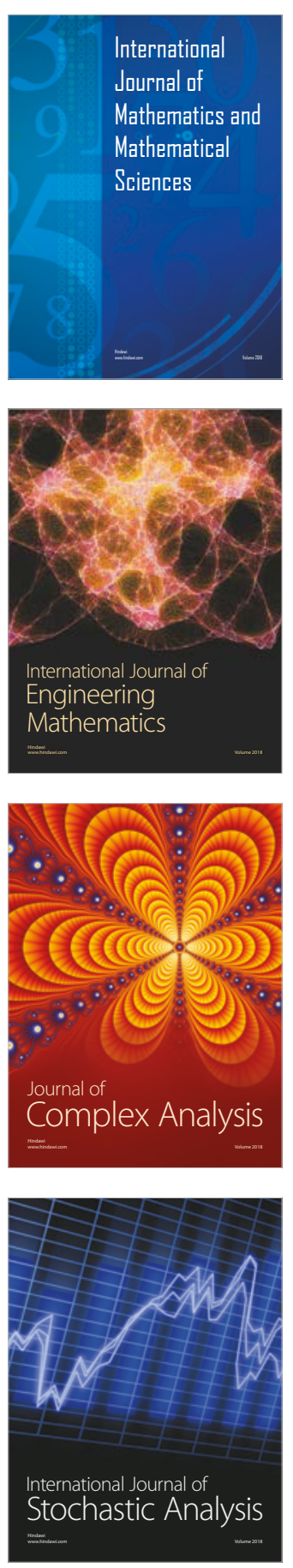
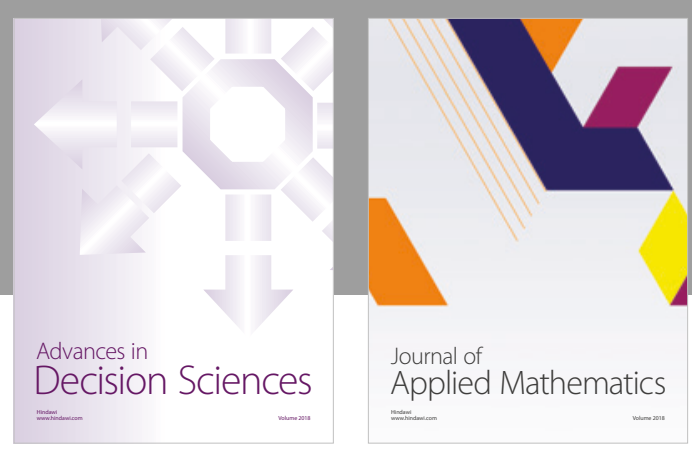

Journal of

Applied Mathematics
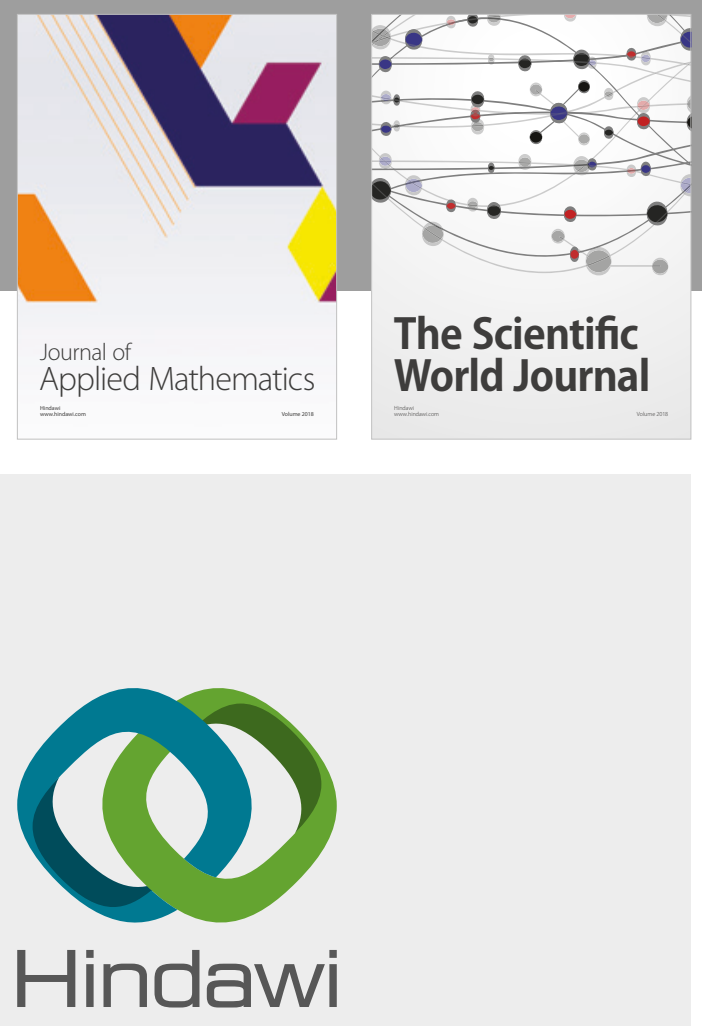

Submit your manuscripts at

www.hindawi.com

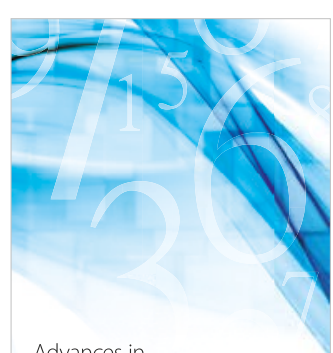

Advances in
Numerical Analysis
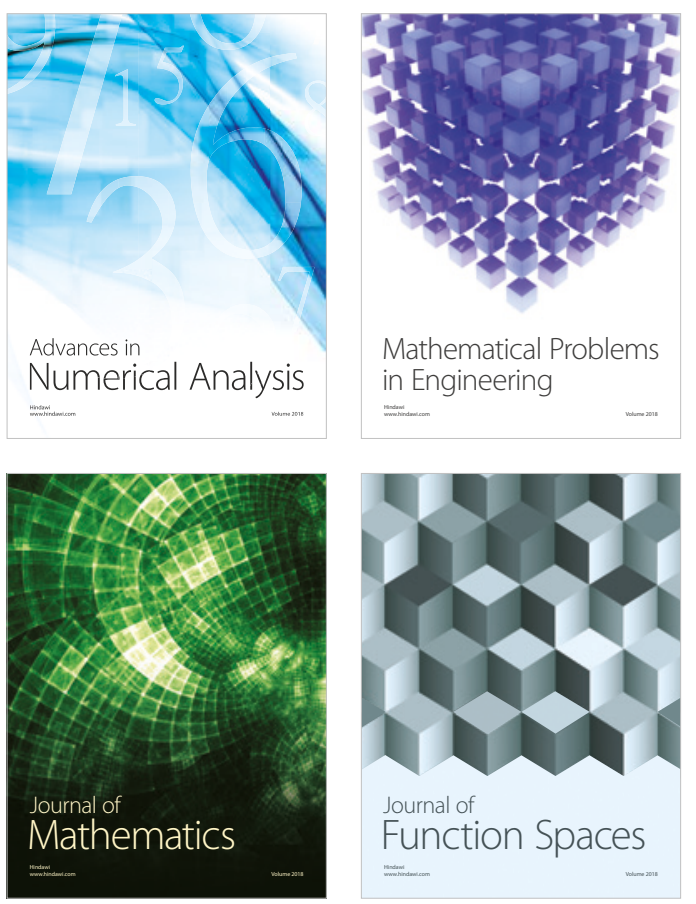

Mathematical Problems in Engineering

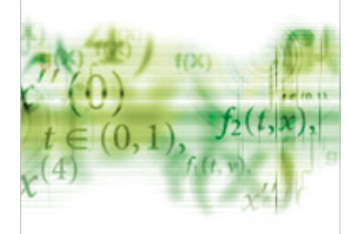

International Journal of

Differential Equations

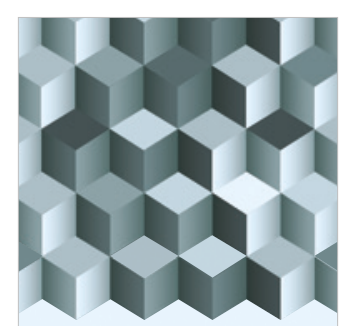

Journal of

Function Spaces
The Scientific

World Journal

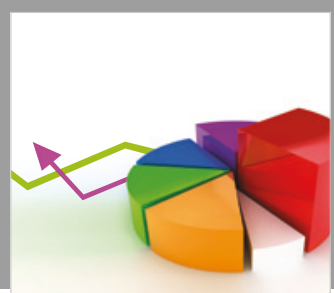

Journal of

Probability and Statistics
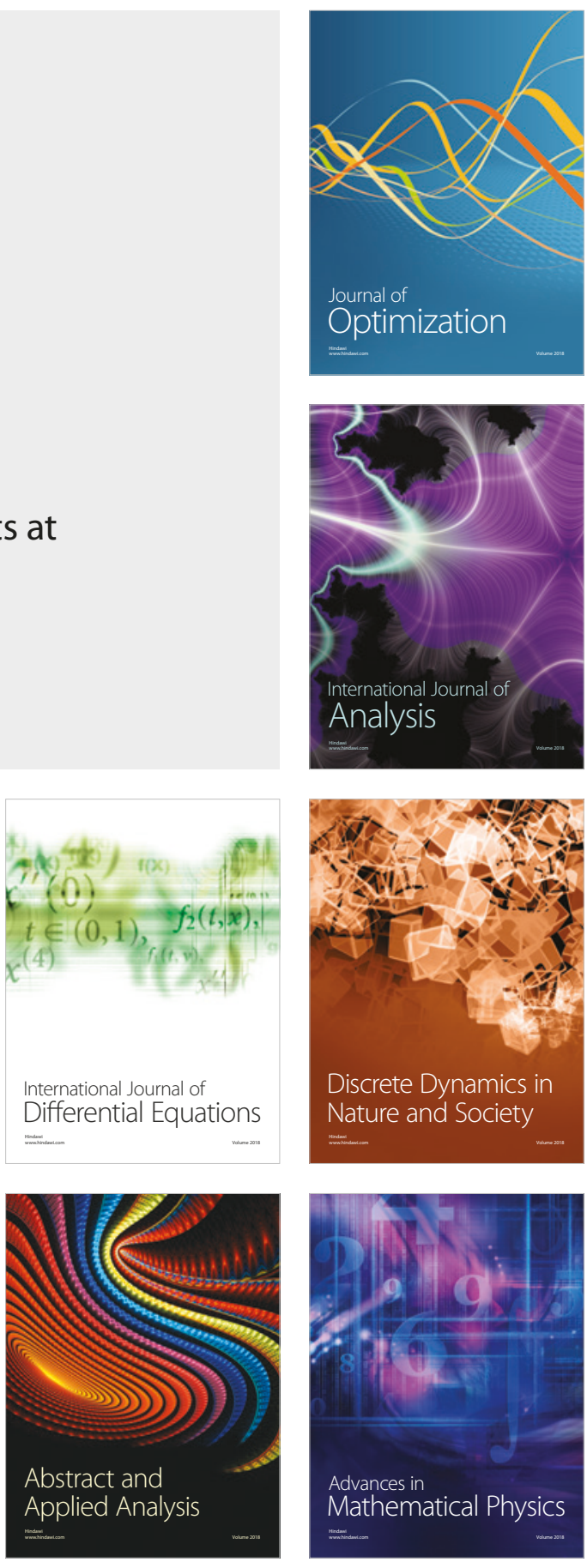